\title{
Grandes estruturas lineares em conjuntos de funções patológicas
}

\author{
Renan Gava de Souza \\ DisSERTAÇÃO APRESENTADA \\ $\mathrm{AO}$ \\ Instituto DE MATEMÁtica e EstatísticA \\ DA \\ Universidade DE SÃo PAUlo \\ PARA \\ OBTENÇÃO DO TÍTULO \\ DE \\ Mestre em Matemática \\ Programa: Mestrado em Matemática \\ Orientador: Prof. Dr. Leonardo Pellegrini Rodrigues
}

São Paulo, Julho de 2019 


\section{Grandes estruturas lineares em conjuntos de funções patológicas}

Esta versão da dissertação contém as correções e alterações sugeridas pela Comissão Julgadora durante a defesa da versão original do trabalho, realizada em 20/05/2019. Uma cópia da versão original está disponível no

Instituto de Matemática e Estatística da Universidade de São Paulo.

Comissão Julgadora:

- Prof. Dr. Leonardo Pellegrini Rodrigues - IME-USP

- Prof. Dr. Thiago Grando - IME-USP

- Prof. Dr. Leandro Cândido Batista - UNIFESP 


\section{Resumo}

SOUZA, R. G. Grandes estruturas lineares em conjuntos de funções patológicas. 2019. Dissertação (Mestrado) - Instituto de Matemática e Estatística, Universidade de São Paulo, São Paulo, 2019.

A busca por grandes estruturas lineares em conjuntos de funções com propriedades patológicas é um tópico que fora desenvolvido nos últimos vinte anos. Esse trabalho detalha alguns desses resultados sobre lineabilidade e espaçabilidade de forma clara e diluida para facilitar a introdução desses conceitos para um pesquisador não familiarizado.

Veremos que os seguintes conjuntos são lineáveis: funções $C^{\infty}$ não analíticas, funções com apenas uma quantidade finita de pontos de continuidade, funções cujas derivadas são ilimitadas num intervalo fechado, funções sobrejetoras em todo lugar que se anulam quase sempre. Também mostraremos a espaçabilidade dos seguintes conjuntos: funções de variação limitada com um conjunto denso de descontinuidades em salto e funções Lebesgue integráveis em [0,1] não essencialmente limitadas em nenhum intervalo. Finalmente, veremos alguns resultados sobre a lineabilidade no conjunto dos funcionais lineares que atingem a norma.

Palavras-chave: Lineabilidade; Espaçabilidade; Funções aditivas; Funcionais que atingem a norma; Funções de variação limitada; Funções Lebesgue integráveis. 


\section{Abstract}

SOUZA, R. G. Large linear structures in sets of pathological functions. 2019. Dissertação (Mestrado) - Instituto de Matemática e Estatística, Universidade de São Paulo, São Paulo, 2019.

Finding large linear structures in sets of functions with pathological properties is a topic that has been developed in the last twenty years. This work details some of these results about lineability and spaceability in a clear and diluted way to make the introduction of these concepts easier for an unfamiliar researcher.

We show that the following sets are lineable: $C^{\infty}$ non-analytic functions, functions with a finite number of points of continuity, functions whose derivative is unbounded on a closed interval and everywhere surjective functions that are almost everywhere zero. We also show the spaceability of the following sets: functions of bounded variation which have a dense set of jump discontinuities and Lebesgue integrable functions in [0,1] which are nowhere essentially bounded. At last, we show some results about lineability in the set of linear functionals that attain their norm.

Keywords: Lineability; Spaceability; Additive functions; Norm-attain functionals; Functions of bounded variation; Lebesgue integrable functions. 


\section{Sumário}

Lista de Símbolos $\quad$ vii

$\begin{array}{ll}\text { Introdução } & 1\end{array}$

1 Conceitos Básicos $\quad 3$

1.1 Espaços Normados . . . . . . . . . . . . . . . . . . . . 3

1.2 Operadores lineares limitados . . . . . . . . . . . . . . . . 5

1.3 Funções de variação limitada . . . . . . . . . . . . . . . . . . . . . . . . . . 7

1.4 Espaços com Base de Schauder . . . . . . . . . . . . . . . . . . . . . 13

2 Conjuntos lineáveis $\quad 27$

2.1 Funções $C^{\infty}$ não analíticas . . . . . . . . . . . . . . . . . . . . . 27

2.2 Funções com apenas uma quantidade finita de pontos de continuidade . . . . 32

2.3 Funções cujas derivadas são ilimitadas num intervalo fechado . . . . . . . . . 34

2.4 Funções sobrejetoras em todo lugar que se anulam quase sempre . . . . . . . 35

3 Conjuntos espaçáveis $\quad 39$

3.1 Funções de variação limitada com um conjunto denso de descontinuidades . . 39

3.2 Funções Lebesgue integráveis não essencialmente limitadas em nenhum intervalo 47

4 Funcionais $\quad 51$

4.1 Funções aditivas descontínuas e lineares . . . . . . . . . . . . . . . . 51

4.2 Funcionais lineares que atingem a norma . . . . . . . . . . . . . . 55

$\begin{array}{ll}\text { Referências Bibliográficas } & 61\end{array}$ 


\section{Lista de Símbolos}

$\begin{array}{ll}\mathbb{N} & \text { Conjunto dos números naturais } \\ \mathbb{Z} & \text { Conjunto dos números inteiros } \\ \mathbb{Q} & \text { Conjunto dos números racionais } \\ \mathbb{R} & \text { Conjunto dos números reais } \\ \mathbb{R}_{+} & \text {Conjunto dos números reais positivos } \\ \mathbb{C} & \text { Conjunto dos números complexos } \\ \operatorname{span}\{A\} & \text { Espaço vetorial gerado pelo conjunto } A \\ B_{X} & \{x \in X:\|x\| \leq 1\} \\ S_{X} & \{x \in X:\|x\|=1\} \\ \aleph_{0} & \text { Cardinal de } \mathbb{N} \\ \mathfrak{c} & \text { Cardinal de } \mathbb{R}\end{array}$


viii LISTA DE SÍMBOLOS 


\section{Introdução}

Funções contínuas diferenciáveis em nenhum ponto, funções contínuas monótonas em nenhum intervalo ou funções sobrejetoras em qualquer intervalo; um exemplo concreto de uma das funções acima não é intuitivo dadas as características peculiares que possuem. No entanto, essas funções existem (por exemplo o Monstro de Weierstrass é uma função contínua não diferenciável em todo ponto) e ao observar a dificuldade na descoberta de tais exemplos, tendemos a achar que devem haver poucas funções com tais particularidades. Porém, demonstraram-se resultados em que conjuntos de funções com essas patologias podem, na verdade, conter espaços vetoriais de dimensão infinita. O estudo de conjuntos com tais estruturas vem se desenvolvendo nos últimos vinte anos aproximadamente e, em [5], V.I. Gurariy introduziu a terminologia de conjuntos lineáveis e espaçáveis.

Um subconjunto $S$ de espaço vetorial $X$ é chamado lineável se $S \cup\{0\}$ contém um subespaço de dimensão infinita. Se $X$ for um espaço topológico e este subespaço for fechado então diremos que $S$ é espaçável. Um dos primeiros resultados nesse tema foi provado por V.I Gurariy em [12] que mostra a lineabilidade do conjunto das funções em [0,1] contínuas não diferenciáveis em nenhum ponto. Mais tarde, junto a Fonf e Kadec, Gurariy mostrou em [6] que este conjunto também é espaçável. Também em [12] foi mostrado que o conjunto das funções contínuas diferenciáveis, apesar de lineável, não é espaçável. Em resultados mais recentes Aron, Seoane-Sepúlveda e Gurariy demonstraram que os conjuntos das funções monótonas em nenhum intervalo e das funções sobrejetoras em todo intervalo são lineáveis no artigo [2].

Na última década, Garcia-Pacheco, Martín e Seoane-Sepúlveda apresentaram em [8] uma coleção de conjuntos lineáveis, espaçáveis ou algebráveis ${ }^{1}$ de funções patológicas. Alguns anos depois, Acosta, Aizpuru, Aron e Garcia-Pacheco provaram que o conjunto dos funcionais lineares que atingem a norma é lineável (ver [1]). Há alguns anos Głab, Kaufmann e Pellegrini demonstraram em [11] a espaçabilidade dos conjuntos das funções de variação limitada com uma coleção densa de descontinuidades e das funções Lebesgue integráveis em [0,1] não essencialmente limitadas em nenhum intervalo. Neste trabalho abordaremos os resultados citados nesse parágrafo.

\footnotetext{
${ }^{1}$ Um conjunto $S$ é algebrável se $S \cup\{0\}$ contém uma álgebra infinitamente gerada. Esse conceito foi introduzido mais recentemente em [3].
} 
No primeiro capítulo veremos algumas noções preliminares essenciais para a compreensão das demonstrações que serão apresentadas dos capítulos seguintes. Nesse capítulo, recordaremos nas primeiras seções alguns conceitos como norma e espaços normados, e operadores limitados. A maioria dos resultados dessas seções é deixada nas referências por serem consideradas básicas. Na Seção 1.3 miraremos em resultados relativos às funções de variação limitada. Essa seção é importante para a leitura da Seção 3.1 adiante. Na última seção desse capítulo o foco serão os espaços com Base de Schauder. Os resultados apresentados aqui serão substanciais nos capítulos 3 e 4.

O Capítulo 2 tratará da lineabilidade de alguns conjuntos de funções reais. Cada seção deste capítulo terá um desses conjuntos como protagonista. Primeiramente falaremos do conjunto das funções $C^{\infty}$ não analíticas. Em seguida veremos o conjunto das funções com apenas uma quantidade finita de pontos de continuidade. Na seção seguinte o foco é o conjunto das funções com derivada ilimitada num intervalo fechado. E finalizaremos com o interessante conjunto das funções sobrejetoras em todo intervalo que se anulam quase sempre.

Os conjuntos apresentados no Capítulo 3 são exemplos de conjuntos espaçáveis de funções reais. Aqui veremos dois resultados. O primeiro é a demonstração que o conjunto das funções de variação limitada com um conjunto denso de descontinuidades em salto é espaçável. E o segundo é a espaçabilidade do conjunto das funções Lebesgue integráveis não essencialmente limitadas em nenhum intervalo.

No último capítulo falaremos sobre alguns resultados de lineabilidade em conjuntos de funcionais lineares. Na análise funcional é comum o uso de funcionais contínuos. Na primeira seção desse capítulo o foco será os conjuntos de funções aditivas descontínuas e o conjunto de funcionais lineares descontínuos. E na última seção veremos dois teoremas sobre condições que o conjunto dos funcionais que atingem a norma devem satisfazer para que sejam lineáveis.

A divisão dos capítulos foi feita desse modo para exibir a proximidade nos métodos usados nas demonstrações. Por exemplo, no Capítulo 2 as demonstrações utilizam conceitos mais algébricos enquanto nos capítulos 3 e 4, onde a noção topológica é mais importante, utilizam-se as bases de Schauder de caráter mais analítico.

Em algumas partes do trabalho assumimos a familiaridade do leitor com alguns conceitos do cálculo, álgebra linear e medida. 


\section{Capítulo 1}

\section{Conceitos Básicos}

Apresentaremos aqui alguns conceitos introdutórios sobre espaços normados, operadores lineares e bases de Schauder. Assumiremos que algumas noções de topologia e álgebra linear já são familiares ao leitor. Consideraremos também que nos momentos em que for citado um corpo $\mathbb{K}$, estaremos nos referindo aos conjuntos $\mathbb{R}$ ou $\mathbb{C}$.

\subsection{Espaços Normados}

Ao longo do trabalho nos depararemos com sequências, limites, funções contínuas entre outras definições em que a ideia de distância é essencial. Introduziremos portanto o conceito de norma.

Definição 1.1.1. Seja $X$ um espaço vetorial sobre $\mathbb{K}$. Uma função $\|\cdot\|: X \rightarrow \mathbb{R}$ é uma norma se:

(i) $\|x\| \geq 0$, para todo $x \in X$;

(ii) $\|x\|=0 \Longleftrightarrow x=0$;

(iii) $\|\lambda x\|=|\lambda|\|x\|$, para todo $x \in X$ e $\lambda \in \mathbb{R}$;

(iv) $\|x+y\| \leq\|x\|+\|y\|$, para todo $x, y \in X$.

Um espaço normado é um espaço vetorial no qual uma norma está definida.

Num espaço normado, a função $d: X \rightarrow \mathbb{R}$ definida por $d(x, y)=\|x-y\|$, para todo $x, y \in X$ é uma métrica sobre $X$, chamada métrica induzida pela norma. Deste modo, todo espaço normado é um espaço métrico.

Considere $(X, d)$ um espaço normado onde $d$ é a métrica induzida pela norma. Uma sequência $\left(x_{n}\right) \subset X$ converge se, existe $x \in X$ tal que para todo $\epsilon>0$ existe $n_{\epsilon}=n(\epsilon) \in \mathbb{N}$ tal que

$$
\left\|x_{n}-x\right\|<\epsilon, \text { para todo } n>n_{\epsilon}
$$


Nesse caso, $x$ é o limite $\left(x_{n}\right)$ e denotaremos por $\lim _{n \rightarrow+\infty} x_{n}=x$ ou $x_{n} \underset{n \rightarrow+\infty}{\longrightarrow} x$. Num espaço normado $X$ isso é equivalente a dizer que a sequência $\left(x_{n}-x\right)$ converge para 0 .

Dizemos que $\left(x_{n}\right) \subset X$ é uma sequência Cauchy se para todo $\epsilon>0$ existe $n_{\epsilon}=n(\epsilon) \in \mathbb{N}$ tal que

$$
\left\|x_{m}-x_{n}\right\|<\epsilon \text {, para todo } m, n>n_{\epsilon} .
$$

Em espaços métricos, toda sequência convergente é sequência de Cauchy. No entanto, existem espaços métricos onde sequências de Cauchy não são convergentes. Por exemplo, a sequência $x_{n}:=\frac{1}{n}$ para todo $n \geq 1$, é uma sequência de Cauchy em $((0,1),|\cdot|)$ que não é convergente. Um espaço métrico $X$ é completo se toda sequência de Cauchy de elementos de $X$ converge. Um espaço de Banach é um espaço normado completo.

Lembremos que em um espaço normado $X$, uma série $\sum_{i=1}^{\infty} x_{i}$ é a sequência das somas finitas $\left(\sum_{i=1}^{n} x_{i}\right)_{n}$. Se o limite dessa sequência existir diremos que a série é convergente. Caso contrário diremos que a série é divergente. Segue da definição de sequência de Cauchy e da completude do conjunto $\mathbb{R}$ o Critério de Cauchy para séries num espaço de Banach. Esse critério diz que, num espaço de Banach, uma série $\sum_{n=1}^{\infty} x_{n}$ é convergente, se e somente se, dado $\epsilon>0$ existe $n_{0} \in \mathbb{N}$ tal que

$$
\left\|\sum_{n=j}^{l} x_{n}\right\|<\epsilon, \text { sempre que } l>j \geq n_{0} .
$$

Num espaço normado as operações de soma e produto por escalar, assim como a norma tem a seguinte propriedade.

Proposição 1.1.2. Seja X um espaço normado. Então as seguintes funções são contínuas.

(i) $+: X \times X \rightarrow X$ definida por $+(x, y)=x+y$;

(ii) $\bullet: X \times \mathbb{K} \rightarrow X$ definida por $\bullet(x, \lambda)=\lambda x$;

(iii) $\|\cdot\|: X \rightarrow \mathbb{K}$ definida por $\|\cdot\|(x)=\|x\|$.

Demonstração. Ver [16], páginas 17 e 18, proposições 1.3.1 e 1.3.2.

Sejam $X$ um espaço normado e $Y$ um subconjunto de $X$. O fecho de $Y$ é o conjunto dos pontos $x \in X$ tais que existe uma sequência $\left(y_{n}\right) \subset Y$ que converge para $x$. O fecho de um subespaço de um espaço normado é um subespaço, como mostra o seguinte resultado.

Proposição 1.1.3. Seja $X$ um espaço normado. Se $U$ é um subespaço vetorial de $X$ então $\bar{U}$ é um subespaço vetorial de $X$.

Demonstração. Ver [16], página 21, Teorema 1.3.10. 
Lembremos que um subconjunto $F$ de um espaço normado $X$ é fechado se, e somente se, $F=\bar{F}$. Segue então outro resultado envolvendo subespaços:

Proposição 1.1.4. Um subespaço $U$ de um espaço de Banach $X$ é completo se, e somente se, $U$ é um conjunto fechado em $X$.

Demonstração. Ver [13], página 67, Teorema 2.3-1.

Duas normas $\|\cdot\|_{1}$ e $\|\cdot\|_{2}$, definidas sobre um mesmo espaço $X$ são equivalentes se elas induzem a mesma topologia. Isto é, os abertos em $X$ com a norma $\|\cdot\|_{1}$ são os mesmos que os abertos em $X$ com a norma $\|\cdot\|_{2}$. Nessas condições os conceitos topológicos que valem para uma norma também valem para a outra. Para verificarmos que duas normas são equivalentes usaremos a seguinte.

Proposição 1.1.5. Duas normas $\|\cdot\|_{1} e\|\|_{2}$ num espaço vetorial $X$ são equivalentes se, $e$ somente se, a aplicação identidade $I:\left(X,\|\cdot\|_{1}\right) \rightarrow\left(X,\|\cdot\|_{2}\right)$ é contínua.

Demonstração. Ver [16], página 45, Corolário 1.6.8.

O seguinte resultado dá uma caracterização dos espaços normados de dimensão finita por meio da compacidade da bola unitária.

Teorema 1.1.6. Um espaço normado $X$ tem dimensão finita se, e somente se, a bola fechada unitária $B_{X}=\{x \in X:\|x\| \leq 1\}$ é um conjunto compacto.

Demonstração. Ver [13], Teoremas 2.5-3 e 2.5-5 nas páginas 77, 78 e 80.

\subsection{Operadores lineares limitados}

Introduziremos algumas notações que serão usadas nessa seção e que também aparecerão nos outros capítulos. Num espaço normado $X$ definiremos os subconjuntos $B_{X}:=\{x \in X$ : $\|x\| \leq 1\}$ e $S_{X}:=\{x \in X:\|x\|=1\}$. Denominaremos esses conjuntos por bola fechada de raio 1 e centro na origem e esfera unitária, respectivamente.

Sejam $X, Y$ espaços vetoriais. Uma função $T: X \rightarrow Y$ é um operador linear se

$$
T(\lambda x+y)=\lambda T(x)+T(y), \text { para todo } x, y \in X \text { e todo escalar } \lambda \in \mathbb{K} \text {. }
$$

Sejam $X$ e $Y$ espaços normados. Um operador linear $T: X \rightarrow Y$ é limitado se existe $C>0$ tal que

$$
\|T(x)\| \leq C\|x\| \text {, para todo } x \in X .
$$

O conjunto dos operadores lineares limitados entre $X$ e $Y$ será denotado por $\mathcal{L}(X, Y)$. Quando $Y=\mathbb{K}, \mathcal{L}(X, \mathbb{K})=X^{*} \cdot \mathcal{L}(X, Y)$ é um espaço vetorial com as operações usuais 
de soma e produto entre operadores. É possível definir uma norma para esse espaço. Seja $T \in \mathcal{L}(X, Y)$. A norma de $T$ é dada por:

$$
\begin{aligned}
\|T\| & :=\sup \left\{\|T(x)\|: x \in S_{X}\right\} \\
& =\sup \left\{\|T(x)\|: x \in B_{X}\right\} \\
& =\inf \{M>0:\|T(x)\| \leq M\|x\|, \text { para todo } x \in X\} .
\end{aligned}
$$

Observamos que se $Y$ é um espaço de Banach então $\mathcal{L}(X, Y)$ é um espaço de Banach. Veja a página 27 de [16] para essa demonstração.

Os operadores lineares limitados se relacionam com operadores lineares contínuos por meio do seguinte teorema.

Teorema 1.2.1. Sejam $X, Y$ espaços normados e $T: X \rightarrow Y$ um operador linear. Então são equivalentes as seguintes afirmações:

(i) Té contínuo;

(ii) Té contínuo em 0;

(iii) T é operador limitado.

Demonstração. Ver [16], página 25, Teorema 1.4.2.

Seja $X$ um espaço normado e $Y$ um subconjunto de $X$. Dizemos que $Y$ é denso em $X$ se $\bar{Y}=X$. No caso de operadores limitados definidos num subespaço de Banach denso o seguinte teorema nos garante a existência de uma extensão para o espaço inteiro.

Teorema 1.2.2. Sejam $D$ um subespaço denso de um espaço normado $X, Y$ um espaço de Banach, e $T_{0}: D \rightarrow Y$ um operador linear limitado. Então existe um único operador linear limitado $T: X \rightarrow Y$ tal que $\left.T\right|_{D}=T_{0}$ e $\|T\|=\left\|T_{0}\right\|$.

Demonstração. Ver [16], páginas 70 e 71, Teorema 1.9.1.

Finalmente, um teorema fundamental na análise funcional. Esse resultado associa a sobrejetividade de um operador com o seu comportamento topológico e será usado na próxima seção.

Teorema 1.2.3. (da Aplicação Aberta) Sejam $X, Y$ são espaços de Banach e $A \in L(X, Y)$ sobrejetora. Então $A$ é uma aplicação aberta (Isto é, para todo aberto $U \subset X$, a imagem $A(U) \subset Y$ é aberto).

Demonstração. Ver [16], páginas 43 e 44, Teorema 1.6.5. 


\subsection{Funções de variação limitada}

Esta seção é uma base para o resultado que será apresentado na Seção 3.1. Aqui veremos alguns conceitos e resultados sobre funções de variação limitada que serão essenciais na demonstração do Teorema 3.1.3.

Uma partição de um intervalo $[a, b] \subset \mathbb{R}$ é um conjunto finito $\left\{x_{1}, \ldots, x_{k}\right\} \subset[a, b]$ tal que

$$
a=x_{1}<x_{2}<\ldots<x_{k-1}<x_{k}=b
$$

Dada uma função $f:[a, b] \rightarrow \mathbb{R}$, em que $a, b \in \mathbb{R}$, a variação total de $f$ num intervalo $I \subset[a, b]$ é definida por:

$$
V_{I}(f)=\sup _{P} \sum_{x_{i} \in P}\left|f\left(x_{i}\right)-f\left(x_{i-1}\right)\right|,
$$

na qual $P$ é uma partição de $I$. Para facilitar a notação, se $I=[a, b]$ escreveremos apenas $V(f)$.

Veremos agora algumas propriedades da variação total que nos ajudarão a entender como ela se comporta em relação as funções.

Proposição 1.3.1. Sejam $f, g:[a, b] \rightarrow \mathbb{R} e c \in \mathbb{R}$. Então

(i) $V(f)=0 \Longleftrightarrow f$ é constante;

(ii) $V(f+g) \leq V(f)+V(g)$;

(iii) $V(c f)=|c| V(f)$;

(iv) $V_{[a, b]}(f)=V_{[a, c]}(f)+V_{[c, b]}(f)$, para $c \in[a, b]$.

Demonstração. (i) Para a primeira implicação usaremos a contra-positiva. Então suponha que $f$ não é constante. Então exitem dois pontos $y, z \in[a, b]$ tais que $y<z$ e $f(y) \neq$ $f(z)$. Considere a partição $\left\{x_{1}=a, x_{2}=y, x_{3}=z, x_{4}=b\right\}$. Assim,

$$
\begin{aligned}
0 & <|f(z)-f(y)| \\
& \leq \sum_{i=1}^{4}\left|f\left(x_{i+1}\right)-f\left(x_{i}\right)\right| \\
& \leq \sup _{P} \sum_{x_{i} \in P}\left|f\left(x_{i+1}\right)-f\left(x_{i}\right)\right|=V(f)
\end{aligned}
$$

Logo, $V(f)>0$. Pela contra-positiva concluímos que se $V(f)=0$ então $f$ é constante. A outra implicação é direta. 
(ii) Seja $P=\left\{x_{1}, \ldots, x_{n}\right\}$ uma partição de $[a, b]$, então

$$
\begin{aligned}
\sum_{i=1}^{n-1}\left|(f+g)\left(x_{i+1}\right)-(f+g)\left(x_{i}\right)\right| & =\sum_{i=1}^{n-1}\left|f\left(x_{i+1}\right)+g\left(x_{i+1}\right)-f\left(x_{i}\right)-g\left(x_{i}\right)\right| \\
& =\sum_{i=1}^{n-1}\left|f\left(x_{i+1}\right)-f\left(x_{i}\right)+g\left(x_{i+1}\right)-g\left(x_{i}\right)\right| \\
& \leq \sum_{i=1}^{n-1}\left|f\left(x_{i+1}\right)-f\left(x_{i}\right)\right|+\left|g\left(x_{i+1}\right)-g\left(x_{i}\right)\right| \\
& =\sum_{i=1}^{n-1}\left|f\left(x_{i+1}\right)-f\left(x_{i}\right)\right|+\sum_{i=1}^{n-1}\left|g\left(x_{i+1}\right)-g\left(x_{i}\right)\right| \\
& \leq \sup _{P} \sum_{x_{i} \in P}\left|f\left(x_{i+1}\right)-f\left(x_{i}\right)\right|+\sup _{P} \sum_{x_{i} \in P}\left|g\left(x_{i+1}\right)-g\left(x_{i}\right)\right| \\
& =V(f)+V(g) .
\end{aligned}
$$

Pela arbitrariedade de $P$, essa desigualdade vale para toda partição de $[a, b]$. Assim podemos tomar o supremo sobre todas as partições no primeiro somatório. Logo

$$
V(f+g)=\sup _{P} \sum_{x_{i} \in P}\left|(f+g)\left(x_{i+1}\right)-(f+g)\left(x_{i}\right)\right| \leq V(f)+V(g) .
$$

(iii) $V(c f)=\sup _{P} \sum_{x_{i} \in P}\left|(c f)\left(x_{i+1}\right)-(c f)\left(x_{i}\right)\right|=\sup _{P} \sum_{x_{i} \in P}\left|c\left(f\left(x_{i+1}\right)-f\left(x_{i}\right)\right)\right|$

$$
=\sup _{P}|c| \sum_{x_{i} \in P}\left|f\left(x_{i+1}\right)-f\left(x_{i}\right)\right|=|c| \sup _{P} \sum_{x_{i} \in P}\left|f\left(x_{i+1}\right)-f\left(x_{i}\right)\right|=|c| V(f) .
$$

(iv) Sejam $P=\left\{x_{1}, \ldots, x_{k}\right\}$ e $Q=\left\{y_{1}, \ldots, y_{k}\right\}$ partições quaisquer de $[a, c]$ e $[c, b]$ respectivamente. Portanto $x_{k}=y_{1}=c$. Logo $P \cup Q$ é uma partição do intervalo $[a, b]$. Segue

$$
\sum_{i=1}^{n-1}\left|f\left(x_{i+1}\right)-f\left(x_{i}\right)\right|+\sum_{i=1}^{m-1}\left|f\left(y_{i+1}\right)-f\left(y_{i}\right)\right| \leq V_{[a, b]}(f) .
$$

Como $P$ e $Q$ são partições arbitrárias vale a desigualdade

$$
\sup _{P} \sum_{i=1}^{n-1}\left|f\left(x_{i+1}\right)-f\left(x_{i}\right)\right|+\sup _{Q} \sum_{i=1}^{m-1}\left|f\left(y_{i+1}\right)-f\left(y_{i}\right)\right| \leq V_{[a, b]}(f) .
$$

Logo

$$
V_{[a, c]}(f)+V_{[c, b]}(f) \leq V_{[a, b]}(f) .
$$

Para a outra desigualdade, seja $P=\left\{x_{1}, \ldots, x_{n}\right\}$ uma partição de $[a, b]$. Existe $m \in \mathbb{N}$ tal que $x_{m-1} \leq c \leq x_{m}$. Então $P_{1}=\left\{x_{1}, \ldots, x_{m-1}, c\right\}$ e $P_{2}=\left\{c, x_{m+1}, \ldots, x_{n}\right\}$ são 
partições de $[a, c]$ e $[c, b]$ respectivamente. Logo

$$
\begin{aligned}
\sum_{i=1}^{n-1}\left|f\left(x_{i+1}\right)-f\left(x_{i}\right)\right| & =\sum_{i=1}^{m-2}\left|f\left(x_{i+1}\right)-f\left(x_{i}\right)\right|+\left|f\left(x_{m}\right)+(f(c)-f(c))-f\left(x_{m-1}\right)\right| \\
& +\sum_{i=m}^{n-1}\left|f\left(x_{i+1}\right)-f\left(x_{i}\right)\right| \\
& \left.\leq \sum_{i=1}^{m-2}\left|f\left(x_{i+1}\right)-f\left(x_{i}\right)\right|+\left|f\left(x_{m}\right)-f(c)\right|+\mid f(c)\right)-f\left(x_{m-1}\right) \mid \\
& +\sum_{i=m}^{n-1}\left|f\left(x_{i+1}\right)-f\left(x_{i}\right)\right| \\
& \leq \sup _{P_{1}}\left\{\sum_{i=1}^{m-2}\left|f\left(x_{i+1}\right)-f\left(x_{i}\right)\right|+\left|f\left(x_{m}\right)-f(c)\right|\right\} \\
& \left.+\sup _{P_{2}}\{\mid f(c))-f\left(x_{m-1}\right)\left|+\sum_{i=m}^{n-1}\right| f\left(x_{i+1}\right)-f\left(x_{i}\right) \mid\right\} \\
& =V_{[a, c]}(f)+V_{[c, b]}(f) .
\end{aligned}
$$

Se tomarmos o supremo de todas as partições $P$ do intervalo $[a, b]$ obteremos

$$
V_{[a, b]}(f) \leq V_{[a, c]}(f)+V_{[c, b]}(f)
$$

$\operatorname{Logo} V_{[a, b]}(f)=V_{[a, c]}(f)+V_{[c, b]}(f)$.

Se uma função tem variação total finita dizemos que essa função é de variação limitada. O conjunto das funções de variação limitada cujo domínio é o intervalo $[a, b]$, munido das operações usuais de soma e produto por escalar de funções, é um espaço vetorial. De fato os itens $(i i)$ e (iii) da Proposição 1.3.1 nos garantem que para todas $f, g:[a, b] \rightarrow \mathbb{R}$ funções de variação limitada e todo $\lambda \in \mathbb{R}$, a combinação linear $(\lambda f+g)$ tem variação limitada. Denotaremos o espaço das funções de variação limitada no intervalo $[a, b]$ por $B V[a, b]$.

As funções crescentes são um exemplo de função de variação limitada. O próximo resultado nos mostrará o porquê.

Proposição 1.3.2. Se $f:[a, b] \rightarrow \mathbb{R}$, em que $a, b \in \mathbb{R}$, é uma função crescente então

$$
V(f)=f(b)-f(a) .
$$


Demonstração. Seja $P=\left\{x_{1}, \ldots, x_{n}\right\}$ uma partição do intervalo $[a, b]$. Como $f$ é crescente,

$$
\sum_{i=1}^{n-1}\left|f\left(x_{i+1}\right)-f\left(x_{i}\right)\right|=\sum_{i=1}^{n-1}\left(f\left(x_{i+1}\right)-f\left(x_{i}\right)\right)=f\left(x_{n}\right)-f\left(x_{1}\right)=f(b)-f(a) .
$$

Como essa igualdade é válida para toda partição de $[a, b]$ então tomando o supremo temos

$$
\sup _{P} \sum_{i=1}^{n-1}\left|f\left(x_{i+1}\right)-f\left(x_{i}\right)\right|=f(b)-f(a)
$$

Logo $V(f)=f(b)-f(a)$.

Um outro aspecto sobre cada $f \in B V[a, b]$ é que elas são funções limitadas. Isto é, existe $M>0$ tal que $|f(x)| \leq M$, para todo $x \in[a, b]$. Pois se tomarmos $x \in[a, b]$ qualquer e considerarmos a partição $P=\{a, x, b\}$ então

$$
|f(x)-f(a)| \leq|f(x)-f(a)|+|f(b)-f(x)| \leq \sup _{P} \sum_{x_{i} \in P}\left|f\left(x_{i+1}\right)-f\left(x_{i}\right)\right|=V(f) .
$$

E então desenvolvemos

$$
|f(x)|=|f(x)-f(a)+f(a)| \leq|f(x)-f(a)|+|f(a)| \leq V(f)+|f(a)| .
$$

Pela arbitrariedade de $x$, a função $f$ é limitada por $M=|f(a)|+V(f)$.

Esse resultado nos dá a inspiração para usarmos a variação total para definirmos uma norma no espaço $B V[0,1]$. A função $\|\cdot\|_{B V}: B V[0,1] \rightarrow \mathbb{R}$ definida por:

$$
\|f\|_{B V}=|f(0)|+V(f)
$$

é uma norma em $B V[0,1]$. De fato, para $f, g \in B V[0,1]$ e $\lambda \in \mathbb{R}$ vale:

(i) $\|f\|_{B V}=|f(0)|+V(f)$. Como o módulo e a variação total são sempre positivos, então $\|f\|_{B V}$ também é.

(ii) $\|f\|_{B V}=0 \Rightarrow|f(0)|+V(f)=0$. O módulo e a variação total são positivos. Logo essa igualdade é satisfeita somente se $f(0)=V(f)=0$. Pelo item (i) da Proposição1.3.1, fé constante. Como $f(0)=0$, então $f=0$.

(iii) Pelo item (iii) da Proposição 1.3.1, $V(\lambda f)=|\lambda| V(f)$ para toda função $f$ e $\lambda \in \mathbb{R}$. Assim,

$$
\|\lambda f\|_{B V}=|\lambda f(0)|+V(\lambda f)=|\lambda||f(0)|+|\lambda| V(f)=|\lambda|\left((f(0)+V(f))=|\lambda|\|f\|_{B V} .\right.
$$


(iv) Pelo item (ii) da Proposição 1.3.1, $V(f+g) \leq V(f)+V(g)$, para toda função $f, g$. Assim,

$$
\begin{aligned}
\|f+g\|_{B V} & =|(f+g)(0)|+V(f+g) \\
& \leq|f(0)+g(0)|+V(f)+V(g) \\
& \leq|f(0)|+|g(0)|+V(f)+V(g) \\
& =|f(0)|+V(f)+|g(0)|+V(g) \\
& =\|f\|_{B V}+\|g\|_{B V} .
\end{aligned}
$$

A norma $\|\cdot\|_{B V}$ se relaciona com a norma $\|\cdot\|_{\infty}: B V[0,1] \rightarrow \mathbb{R}$ definida por

$$
\|f\|_{\infty}=\sup \{f(x): x \in[0,1]\}
$$

A $\|\cdot\|_{\infty}$ está bem definida pois $f$ é limitada. Veremos essa relação através da seguinte:

Proposição 1.3.3. A função identidade id $:\left(B V[0,1],\|\cdot\|_{B V}\right) \rightarrow\left(B V[0,1],\|\cdot\|_{\infty}\right)$ é contínua.

Demonstração. Tome $h \in B V[0,1]$ tal que $\|h\|_{B V} \leq 1$. Assim, para todo $x \in[0,1]$ temos:

$$
\begin{aligned}
|h(x)| & =|h(x)-h(0)+h(0)| \\
& \leq|h(x)-h(0)|+|h(0)| \\
& \leq|h(0)|+V(f) \\
& =\|h\|_{B V} \leq 1 .
\end{aligned}
$$

Por isso,

$$
\|h\|_{\infty}=\sup \{|h(x): x \in[0,1]|\} \leq 1,
$$

e portanto, $\|i d\| \leq 1$, isto é, $i d$ é contínua.

Por meio desse resultado concluímos toda sequência convergente em $\left(B V[0,1],\|\cdot\|_{B V}\right)$ também converge em $\left(B V[0,1],\|\cdot\|_{\infty}\right)$. Essa ferramenta nos ajudará a mostrar que o espaço $\left(B V[0,1],\|\cdot\|_{B V}\right)$ é completo e, portanto, um espaço de Banach.

Teorema 1.3.4. O espaço normado $\left(B V[0,1],\|.\|_{B V}\right)$ é um espaço de Banach.

Demonstração. Seja $\left(f_{n}\right)$ uma sequência de Cauchy em $\left(B V[0,1],\|\cdot\|_{B V}\right)$. Em particular $\left(f_{n}\right)$ é uma sequência de Cauchy em $\left(B V[0,1],\|\cdot\|_{\infty}\right)$. Portanto para todo $\epsilon>0$, existe $n_{\epsilon}=n(\epsilon) \in \mathbb{N}$ tal que

$$
\left\|f_{j}-f_{l}\right\|_{\infty}<\epsilon, \text { para todo } j, l>n_{\epsilon}
$$


Daí

$$
\begin{aligned}
& \left\|f_{m}-f_{n}\right\|_{\infty}=\sup \left\{\left(f_{j}-f_{l}\right)(x): x \in[0,1]\right\}<\epsilon \\
& \Rightarrow\left(f_{j}-f_{l}\right)(x)<\epsilon, \text { para todo } x \in[0,1] .
\end{aligned}
$$

Logo, $\left(f_{n}(x)\right) \subset \mathbb{R}$ é uma sequência de Cauchy, para todo $x \in[0,1]$. Como $\mathbb{R}$ é completo, cada $\left(f_{n}(x)\right)$ converge. Seja $f:[0,1] \rightarrow \mathbb{R}$ definida por

$$
f(x)=\lim _{n \rightarrow \infty} f_{n}(x)
$$

A função $f$ tem variação limitada. De fato, para $P=\left\{x_{1}, \ldots, x_{k}\right\}$ uma partição de $[0,1]$ qualquer e $\epsilon>0$, existe $n_{\epsilon} \in \mathbb{N}$ tal que $\left\|f_{m}-f_{n}\right\|_{B V}<\epsilon$ para todo $m, n>n_{\epsilon}$. Fixemos $n>n_{\epsilon}$ e observemos que

$$
\begin{aligned}
& \sum_{i=1}^{k-1}\left|\left(f_{m}-f_{n}\right)\left(x_{i+1}\right)-\left(f_{m}-f_{n}\right)\left(x_{i}\right)\right| \leq V\left(f_{m}-f_{n}\right) \\
& \leq\left\|f_{m}-f_{n}\right\|_{B V}<\epsilon, \text { para todo } m>n_{\epsilon} .
\end{aligned}
$$

Ou seja,

$$
\sum_{i=1}^{k-1}\left|\left(f_{m}-f_{n}\right)\left(x_{i+1}\right)-\left(f_{m}-f_{n}\right)\left(x_{i}\right)\right|<\epsilon, \text { para todo } m>n_{\epsilon} .
$$

Agora, vamos ver o que acontece para $f-f_{n}$.

$$
\begin{aligned}
\sum_{i=1}^{k-1}\left|\left(f-f_{n}\right)\left(x_{i+1}\right)-\left(f-f_{n}\right)\left(x_{i}\right)\right| & =\sum_{i=1}^{k-1}\left|\lim _{m \rightarrow \infty}\left(f_{m}-f_{n}\right)\left(x_{i+1}\right)-\lim _{m \rightarrow \infty}\left(f_{m}-f_{n}\right)\left(x_{i}\right)\right| \\
& =\sum_{i=1}^{k-1}\left|\lim _{m \rightarrow \infty}\left(\left(f_{m}-f_{n}\right)\left(x_{i+1}\right)-\left(f_{m}-f_{n}\right)\left(x_{i}\right)\right)\right| .
\end{aligned}
$$

Como a função módulo é contínua podemos tirar o limite para fora. Segue

$$
\begin{aligned}
\sum_{i=1}^{k-1}\left|\left(f-f_{n}\right)\left(x_{i+1}\right)-\left(f-f_{n}\right)\left(x_{i}\right)\right| & =\sum_{i=1}^{k-1} \lim _{m \rightarrow \infty}\left|\left(f_{m}-f_{n}\right)\left(x_{i+1}\right)-\left(f_{m}-f_{n}\right)\left(x_{i}\right)\right| \\
& =\lim _{m \rightarrow \infty} \sum_{i=1}^{k-1}\left|\left(f_{m}-f_{n}\right)\left(x_{i+1}\right)-\left(f_{m}-f_{n}\right)\left(x_{i}\right)\right|
\end{aligned}
$$


A fórmula (1.1) vale para todo $m>n_{\epsilon}$. Portanto

$$
\lim _{m \rightarrow \infty} \sum_{i=1}^{k-1}\left|\left(f_{m}-f_{n}\right)\left(x_{i+1}\right)-\left(f_{m}-f_{n}\right)\left(x_{i}\right)\right| \leq \epsilon .
$$

Ou seja,

$$
\sum_{i=1}^{k-1}\left|\left(f-f_{n}\right)\left(x_{i+1}\right)-\left(f-f_{n}\right)\left(x_{i}\right)\right| \leq \epsilon .
$$

Como $P$ foi tomado arbitrariamente, a inequação (1.2) vale para toda partição de $[0,1]$. Logo

$$
V\left(f-f_{n}\right)=\sup _{p} \sum_{i=1}^{k-1}\left|\left(f-f_{n}\right)\left(x_{i+1}\right)-\left(f-f_{n}\right)\left(x_{i}\right)\right| \leq \epsilon .
$$

Portanto $\left(f-f_{n}\right) \in B V[0,1]$ que é um espaço vetorial. Então $f=\left(f-f_{n}\right)+f_{n} \in B V[0,1]$.

Do que verificamos anteriormente, concluímos que para todo $\epsilon>0$, existe $n_{\epsilon}$ tal que

$$
V\left(f-f_{n}\right)<\frac{\epsilon}{2}, \text { para todo } n>n_{\epsilon}
$$

Como $f_{n}(0)$ converge para $f(0)$ então existe $m_{\epsilon} \in \mathbb{N}$ tal que

$$
\left|f(0)-f_{n}(0)\right|<\frac{\epsilon}{2} \text {, para todo } n>m_{\epsilon} .
$$

Portanto dado $\epsilon>0$, tomemos $N=\max \left\{n_{\epsilon}, m_{\epsilon}\right\}$. Assim

$$
\begin{aligned}
\left\|f-f_{n}\right\|_{B V} & =\left|\left(f-f_{n}\right)(0)\right|+V\left(f-f_{n}\right) \\
& =\left|f(0)-f_{n}(0)\right|+V\left(f-f_{n}\right) \\
& <\frac{\epsilon}{2}+\frac{\epsilon}{2}=\epsilon \text {, para todo } n>N .
\end{aligned}
$$

$\operatorname{Logo}\left(f_{n}\right)$ converge para $f$ em $\left(B V[0,1],\|\cdot\|_{B V}\right)$. Pela arbitrariedade de $\left(f_{n}\right),\left(B V[0,1],\|\cdot\|_{B V}\right)$ é um espaço de Banach.

\subsection{Espaços com Base de Schauder}

Nessa seção apresentaremos alguns conceitos a respeito de Bases de Schauder com o objetivo de demonstrar o Teorema 1.4.11 e resultados envolvendo projeções naturais associadas a uma base de Schauder. Esses resultados serão necessários para melhor entendimento das demonstrações apresentadas no Capítulo 4, enquanto que o 1.4.11 nos ajudará a caracterizar sequências básicas nos capítulos 3 e 4 .

O Lema de Zorn nos garante que todo espaço vetorial possui uma base de Hamel. No 
entanto as bases de Hamel não carregam o caráter topológico do espaço, pois envolvem apenas somas finitas nas decomposições dos elementos. Em espaços de dimensão finita isso não é um problema pois todas as normas são equivalentes e geram a mesma topologia. Porém isso não acontece quando a dimensão do espaço é infinita. As bases de Schauder nos auxiliam a manipular os elementos desses espaços através da convergência de séries e, portanto, a topologia do espaço é levada em consideração. Vamos então a seguinte:

Definição 1.4.1. Uma sequência $\left(x_{n}\right)$ num espaço de Banach $X$ é uma base de Schauder de $X$ se para cada $x \in X$ existe uma única sequência de escalares $\left(\alpha_{n}\right)$ tal que $x=\sum_{n=1}^{\infty} \alpha_{n} x_{n}$. Notemos que a unicidade dos escalares impossibilita que o vetor nulo seja um elemento da base.

Lembremos que um conjunto $A$ de um espaço vetorial é linearmente independente se todo subconjunto finito de $A$ for linearmente independente. Em dimensão finita os conjuntos linearmente independentes são bases para os espaços que geram. Para bases de Schauder teremos algo análogo por meio das sequências básicas:

Definição 1.4.2. Uma sequência $\left(x_{n}\right)$ num espaço de Banach é uma sequência básica se é uma base de Schauder para $\overline{\operatorname{span}\left\{x_{n}: n \in \mathbb{N}\right\}}$.

Observação: Toda base de Schauder de um espaço normado é linearmente independente. Suponha por absurdo que exista uma base de Schauder $\left(x_{n}\right) \subset X$ que não seja linearmente independente. Logo existe $k \in \mathbb{N}$ tal que $\left\{x_{1}, \ldots, x_{k}\right\}$ é linearmente dependente, ou seja, existe $i \in\{1, \ldots, k\}$ tal que $x_{i}=\sum_{n=1}^{i-1} \beta_{n} x_{n}+\sum_{n=i+1}^{k} \beta_{n} x_{n}$, com os $\beta_{n} \in \mathbb{K}$ não todos nulos. Por outro lado, $x_{i}=\sum_{n=1}^{\infty} \alpha_{n} x_{n}$ em que $\alpha_{n}=\delta_{i n}$ para cada $n \in \mathbb{N}$. Isso contraria a existência de uma única sequência de escalares $\left(\alpha_{n}\right)$ tal que $x_{i}=\sum_{n=1}^{\infty} \alpha_{n} x_{n}$. Portanto, toda base de Schauder de um espaço normado é linearmente independente.

Seja $X$ um espaço de Banach com base de Schauder $\left(x_{n}\right)$. Definimos a função $\|\cdot\|_{\left(x_{n}\right)}$ : $X \rightarrow[0,+\infty[$ por:

$$
\|x\|_{\left(x_{n}\right)}=\left\|\sum_{n=1}^{\infty} \alpha_{n} x_{n}\right\|_{\left(x_{n}\right)}=\sup _{m \in \mathbb{N}}\left\|\sum_{n=1}^{m} \alpha_{n} x_{n}\right\|
$$

A função $\|\cdot\|_{\left(x_{n}\right)}$ está bem definida já que as séries envolvidas são convergentes, e portanto suas somas parciais são limitadas. Agora veremos que $\|\cdot\|_{\left(x_{n}\right)}$ é uma norma em $X$. Sejam $x, y \in X$ e $\lambda \in \mathbb{K}$. Como $\left(x_{n}\right)$ é base de Schauder existem únicas sequências de escalares $\left(\alpha_{n}\right)$ e $\left(\beta_{n}\right)$ tais que $x=\sum_{n=1}^{\infty} \alpha_{n} x_{n}$ e $y=\sum_{n=1}^{\infty} \beta_{n} x_{n}$. Logo, 
(i)

$$
\begin{aligned}
\|x\|_{\left(x_{n}\right)}=0 & \Rightarrow \sup _{m \in \mathbb{N}}\left\|\sum_{n=1}^{m} \alpha_{n} x_{n}\right\| \\
& \Rightarrow\left\|\sum_{n=1}^{m} \alpha_{n} x_{n}\right\|=0, \text { para todo } m \in \mathbb{N} \\
& \Rightarrow \sum_{n=1}^{m} \alpha_{n} x_{n}=0, \text { para todo } m \in \mathbb{N} \\
& \Rightarrow x=\sum_{n=1}^{\infty} \alpha_{n} x_{n}=\lim _{m \rightarrow \infty} \sum_{n=1}^{m} \alpha_{n} x_{n}=0 .
\end{aligned}
$$

A recíproca é imediata.

(ii) Para todo $x \in X,\|x\|_{\left(x_{n}\right)}$ é calculada tomando o supremo de um conjunto de números não negativos. Logo, $\|x\|_{\left(x_{n}\right)} \geq 0$ para todo $x \in X$.

$$
\begin{aligned}
\|\lambda x\|_{\left(x_{n}\right)} & =\sup _{m \in \mathbb{N}}\left\|\lambda \sum_{n=1}^{m} \alpha_{n} x_{n}\right\| \\
& =\sup _{m \in \mathbb{N}}|\lambda|\left\|\sum_{n=1}^{m} \alpha_{n} x_{n}\right\| \\
& =|\lambda| \sup _{m \in \mathbb{N}}\left\|\sum_{n=1}^{m} \alpha_{n} x_{n}\right\| \\
& =|\lambda|\|x\|_{\left(x_{n}\right)} .
\end{aligned}
$$

(iv) Para todo $m \in \mathbb{N}$ temos:

$$
\begin{aligned}
\left\|\sum_{n=1}^{m}\left(\alpha_{n}+\beta_{n}\right) x_{n}\right\| & \leq\left\|\sum_{n=1}^{m} \alpha_{n} x_{n}\right\|+\left\|\sum_{n=1}^{m} \beta_{n} x_{n}\right\| \\
& \leq \sup _{m \in \mathbb{N}}\left\|\sum_{n=1}^{m} \alpha_{n} x_{n}\right\|+\sup _{m \in \mathbb{N}}\left\|\sum_{n=1}^{m} \beta_{n} x_{n}\right\| \\
& =\|x\|_{\left(x_{n}\right)}+\|y\|_{\left(x_{n}\right)},
\end{aligned}
$$

e portanto, $\|x+y\|_{\left(x_{n}\right)} \leq\|x\|_{\left(x_{n}\right)}+\|y\|_{\left(x_{n}\right)}$.

Essa nova norma é conveniente pois mantém a completude do espaço inicial.

Teorema 1.4.3. Sejam $(X,\|\cdot\|)$ um espaço de Banach e $\left(x_{n}\right) \subset(X,\|\cdot\|)$ uma base de Schauder. Então:

(a) $\left(X,\|\cdot\|_{\left(x_{n}\right)}\right)$ é um espaço de Banach.

(b) As normas $\|\cdot\| e\|\cdot\|_{\left(x_{n}\right)}$ são equivalentes. 
Demonstração. (a) Seja $\left(y_{j}\right) \subset\left(X,\|\cdot\|_{\left(x_{n}\right)}\right)$ uma sequência de Cauchy. Como $\left(x_{n}\right)$ é base de Schauder de $X$, para cada $j \in \mathbb{N}$, existe uma única sequência de escalares $\left(\alpha_{j, n}\right)$ tal que

$$
y_{j}=\sum_{n=1}^{\infty} \alpha_{j, n} x_{n} .
$$

Como $\left(y_{j}\right) \subset\left(X,\|\cdot\|_{\left(x_{n}\right)}\right)$ é uma sequência de Cauchy, para todo $\epsilon>0$ existe $j_{\epsilon}=j(\epsilon)>0$ tal que

$$
\left\|y_{j}-y_{l}\right\|_{\left(x_{n}\right)}<\frac{\epsilon}{3}, \text { para todo } j, l \geq j_{\epsilon} .
$$

Observe que como $\left(x_{n}\right)$ é base de Schauder, $\left\|x_{n}\right\| \neq 0$, para todo $n \in \mathbb{N}$. Logo, se $k=1$, então:

$$
\begin{aligned}
\left|\alpha_{j, 1}-\alpha_{l, 1}\right|\left\|x_{1}\right\| & =\left\|\left(\alpha_{j, 1}-\alpha_{l, 1}\right) x_{1}\right\| \\
& \leq\left\|\sum_{n=1}^{\infty}\left(\alpha_{j, k}-\alpha_{l, k}\right) x_{n}\right\|_{\left(x_{n}\right)} \\
& =\left\|y_{j}-y_{l}\right\|_{\left(x_{n}\right)}<\frac{\epsilon}{3}, \text { para todo } j, l>n_{\epsilon} .
\end{aligned}
$$

Ou seja,

$$
\left|\alpha_{j, 1}-\alpha_{l, 1}\right|<\frac{\epsilon}{3\left\|x_{1}\right\|}, \text { para todo } j, l>n_{\epsilon} .
$$

Se $k>1$, então

$$
\begin{aligned}
\left|\alpha_{j, k}-\alpha_{l, k}\right|\left\|x_{k}\right\| & =\left\|\left(\alpha_{j, k}-\alpha_{l, k}\right) x_{k}\right\| \\
& =\left\|\sum_{n=1}^{k}\left(\alpha_{j, n}-\alpha_{l, n}\right) x_{n}-\sum_{n=1}^{k-1}\left(\alpha_{j, n}-\alpha_{l, n}\right) x_{n}\right\| \\
& \leq\left\|\sum_{n=1}^{k}\left(\alpha_{j, n}-\alpha_{l, n}\right) x_{n}\right\|+\left\|\sum_{n=1}^{k-1}\left(\alpha_{j, n}-\alpha_{l, n}\right) x_{n}\right\| \\
& \leq \sup _{k \in \mathbb{N}}\left\|\sum_{n=1}^{k}\left(\alpha_{j, n}-\alpha_{l, n}\right) x_{n}\right\|+\sup _{k \in \mathbb{N}}\left\|\sum_{n=1}^{k-1}\left(\alpha_{j, n}-\alpha_{l, n}\right) x_{n}\right\| \\
& =2\left\|\sum_{n=1}^{\infty}\left(\alpha_{j, n}-\alpha_{l, n}\right) x_{n}\right\| \\
& =2\left\|y_{j}-y_{l}\right\|_{\left(x_{n}\right)}<\frac{2 \epsilon}{3}, \text { para todo } j, l>n_{\epsilon} .
\end{aligned}
$$

Ou seja,

$$
\left|\alpha_{j, k}-\alpha_{l, k}\right|<\frac{2 \epsilon}{\left\|x_{k}\right\|}, \text { para todo } j, l>n_{\epsilon}
$$

Isto é, $\left(\alpha_{j, k}\right)$ é sequência de Cauchy para todo $k \in \mathbb{N}$. Como $\mathbb{K}$ é completo, existe $\alpha_{k} \in \mathbb{K}$ 
tal que $\alpha_{k}=\lim _{j \rightarrow \infty} \alpha_{j, k}$, para todo $k \in \mathbb{N}$.

Vamos mostrar, usando o critério de Cauchy para séries num espaço de Banach, que a série $\sum_{n=1}^{\infty} \alpha_{n} x_{n}$ converge em $(X,\|\cdot\|)$. Antes verificaremos algumas estimativas.

(1) Seja $m \in \mathbb{N}$. Considere $j, l>j_{\epsilon}$ satisfazendo a inequação (1.4).

$$
\begin{aligned}
\left\|\sum_{n=1}^{m} \alpha_{j, n} x_{n}-\sum_{n=1}^{m} \alpha_{l, n} x_{n}\right\| & =\left\|\sum_{n=1}^{m}\left(\alpha_{j, n}-\alpha_{l, n}\right) x_{n}\right\| \\
& \leq \sup _{m \in \mathbb{N}}\left\|\sum_{n=1}^{m}\left(\alpha_{j, n}-\alpha_{l, n}\right) x_{n}\right\| \\
& =\left\|\sum_{n=1}^{\infty}\left(\alpha_{j, n}-\alpha_{l, n}\right) x_{n}\right\|_{\left(x_{n}\right)} \\
& =\left\|\sum_{n=1}^{\infty} \alpha_{j, n} x_{n}-\sum_{n=1}^{\infty} \alpha_{l, n} x_{n}\right\|_{\left(x_{n}\right)} \\
& =\left\|y_{j}-y_{l}\right\|_{\left(x_{n}\right)}<\frac{\epsilon}{3} .
\end{aligned}
$$

Fazendo $l \rightarrow \infty$, temos que

$$
\left\|\sum_{n=1}^{m} \alpha_{j_{\epsilon}, n} x_{n}-\sum_{n=1}^{m} \alpha_{n} x_{n}\right\| \leq \frac{\epsilon}{3}, \text { para todo } m \in \mathbb{N} \text { e todo } j \geq j_{\epsilon} .
$$

(2) Sejam $m_{1}, m_{2} \in \mathbb{N}$ tais que $m_{2} \geq m_{1}>1$. Logo:

$$
\begin{aligned}
\left\|\sum_{n=m_{1}}^{m_{2}} \alpha_{j_{\epsilon}, n} x_{n}-\sum_{n=m_{1}}^{m_{2}} \alpha_{n} x_{n}\right\| & =\| \sum_{n=m_{1}}^{m_{2}} \alpha_{j_{\epsilon}, n} x_{n}+\left(\sum_{n=1}^{m_{1}-1} \alpha_{j_{\epsilon}, n} x_{n}-\sum_{n=1}^{m_{1}-1} \alpha_{j_{\epsilon}, n} x_{n}\right) \\
& +\left(\sum_{n=1}^{m_{1}-1} \alpha_{n} x_{n}-\sum_{n=1}^{m_{1}-1} \alpha_{n} x_{n}\right)-\sum_{n=m_{1}}^{m_{2}} \alpha_{n} x_{n} \| \\
& =\|\left(\sum_{n=m_{1}}^{m_{2}} \alpha_{j_{\epsilon}, n} x_{n}+\sum_{n=1}^{m_{1}-1} \alpha_{j_{\epsilon}, n} x_{n}\right)-\sum_{n=1}^{m_{1}-1} \alpha_{j_{\epsilon}, n} x_{n} \\
& +\sum_{n=1}^{m_{1}-1} \alpha_{n} x_{n}-\left(\sum_{n=1}^{m_{1}-1} \alpha_{n} x_{n}+\sum_{n=m_{1}}^{m_{2}} \alpha_{n} x_{n}\right) \| \\
& =\left\|\sum_{n=1}^{m_{2}} \alpha_{j_{\epsilon}, n} x_{n}-\sum_{n=1}^{m_{2}} \alpha_{n} x_{n}-\sum_{n=1}^{m_{1}-1} \alpha_{j_{\epsilon}, n} x_{n}+\sum_{n=1}^{m_{1}-1} \alpha_{n} x_{n}\right\| \\
& \leq\left\|\sum_{n=1}^{m_{2}} \alpha_{j_{\epsilon}, n} x_{n}-\sum_{n=1}^{m_{2}} \alpha_{n} x_{n}\right\|+\left\|\sum_{n=1}^{m_{1}-1} \alpha_{n} x_{n}-\sum_{n=1}^{m_{1}-1} \alpha_{j_{\epsilon}, n} x_{n}\right\| \\
& \leq \frac{\epsilon}{3}+\frac{\epsilon}{3}=\frac{2 \epsilon}{3}, \text { pois }(1.5) \text { é válida para todo } m \in \mathbb{N} .
\end{aligned}
$$


Por outro lado, a série $\sum_{n=1}^{\infty} \alpha_{j_{\epsilon}, n} x_{n}$ converge em $(X,\|\cdot\|)(\operatorname{ver}(1.3))$. Pelo Critério de Cauchy, existe $m_{\epsilon} \in \mathbb{N}$ tal que $\left\|\sum_{n=m_{1}}^{m_{2}} \alpha_{j_{\epsilon}, n} x_{n}\right\|<\frac{\epsilon}{3}$, para todo $m_{2}>m_{1} \geq m_{\epsilon}$.

Logo,

$$
\begin{aligned}
\left\|\sum_{n=m_{1}}^{m_{2}} \alpha_{n} x_{n}\right\| & =\left\|\sum_{n=m_{1}}^{m_{2}} \alpha_{n} x_{n}+\sum_{n=m_{1}}^{m_{2}} \alpha_{j_{\epsilon}, n} x_{n}-\sum_{n=m_{1}}^{m_{2}} \alpha_{j_{\epsilon}, n} x_{n}\right\| \\
& \leq\left\|\sum_{n=m_{1}}^{m_{2}} \alpha_{n} x_{n}+\sum_{n=m_{1}}^{m_{2}} \alpha_{j_{\epsilon}, n} x_{n}\right\|+\left\|\sum_{n=m_{1}}^{m_{2}} \alpha_{j_{\epsilon}, n} x_{n}\right\| \\
& <\frac{2 \epsilon}{3}+\frac{\epsilon}{3}=\epsilon .
\end{aligned}
$$

Novamente, pelo Critério de Cauchy, a série $\sum_{n=1}^{\infty} \alpha_{n} x_{n}$ converge em $(X,\|\cdot\|)$. Isto é, existe $y \in X$ tal que $y=\sum_{n=1}^{\infty} \alpha_{n} x_{n}$.

Por (1.5),

$$
\left\|\sum_{n=1}^{m} \alpha_{j, n} x_{n}-\sum_{n=1}^{m} \alpha_{n} x_{n}\right\| \leq \epsilon, \text { para todo } m \in \mathbb{N} \text { e todo } j \geq j_{\epsilon} .
$$

Assim,

$$
\left\|y_{j}-y\right\|_{\left(x_{n}\right)}=\left\|\sum_{n=1}^{\infty} \alpha_{j, n} x_{n}-\sum_{n=1}^{\infty} \alpha_{n} x_{n}\right\|_{\left(x_{n}\right)}=\sup _{m \in \mathbb{N}}\left\|\sum_{n=1}^{m} \alpha_{j, n} x_{n}-\sum_{n=1}^{m} \alpha_{n} x_{n}\right\| \leq \epsilon .
$$

Ou seja, $\left(y_{j}\right)$ converge para $y$ em $\left(X,\|\cdot\|_{\left(x_{n}\right)}\right)$. Portanto $\left(X,\|\cdot\|_{\left(x_{n}\right)}\right)$ é um espaço de Banach

(b) As normas serão equivalentes pois para $x=\sum_{n=1}^{\infty} \alpha_{n} x_{n}$ um elemento de $X$. Como para todo $m \in \mathbb{N}$ vale $\left\|\sum_{n=1}^{m} \alpha_{n} x_{n}\right\| \leq\|x\|_{\left(x_{n}\right)}$ então:

$$
\lim _{m \rightarrow \infty}\left\|\sum_{n=1}^{m} \alpha_{n} x_{n}\right\| \leq\|x\|_{\left(x_{n}\right)}
$$

e, pela continuidade de $\|\cdot\|,\|x\| \leq\|x\|_{\left(x_{n}\right)}$. Essa desigualdade também nos mostra que a função $I d:\left(X,\|\cdot\|_{\left(x_{n}\right)}\right) \rightarrow(X,\|\cdot\|)$ é contínua entre dois espaços de Banach. Pelo Teorema da Aplicação Aberta, Id é uma aplicação aberta. Assim $I d^{-1}=I d:(X,\|\cdot\|) \rightarrow$ 
$\left(X,\|\cdot\|_{\left(x_{n}\right)}\right)$ é contínua, logo existe $c>0$ tal que

$$
\|x\|_{\left(x_{n}\right)}=\left\|I d^{-1}(x)\right\|_{\left(x_{n}\right)} \leq c\|x\| .
$$

Portanto as duas normas são equivalentes.

Definição 1.4.4. Seja $X$ um espaço de Banach com base de Schauder e $m \in \mathbb{N}$. A função $P_{m}: X \rightarrow X$ definida por $P_{m}\left(\sum_{n=1}^{\infty} \alpha_{n} x_{n}\right)=\sum_{n=1}^{m} \alpha_{n} x_{n}$, é chamada de $m$-ésima projeção natural associada a base $\left(x_{n}\right)$.

O próximo resultado nos mostrará que, assim como as projeções em espaços de dimensão finita, cada uma das projeções naturais é contínua.

Proposição 1.4.5. Para todo $m \in \mathbb{N}, P_{m}$ é uma transformação linear contínua.

Demonstração. Tome $m \in \mathbb{N}$ qualquer. Claramente $P_{m}$ é linear. Agora, para $x \in X$ qualquer, temos:

$$
\left\|P_{m}(x)\right\|=\left\|P_{m}\left(\sum_{n=1}^{\infty} \alpha_{n} x_{n}\right)\right\|=\left\|\sum_{n=1}^{m} \alpha_{n} x_{n}\right\| \leq \sup _{m \in \mathbb{N}}\left\|\sum_{n=1}^{m} \alpha_{n} x_{n}\right\| \leq\|x\|_{\left(x_{n}\right)} \leq c\|x\|,
$$

onde a última desigualdade vem da equivalência das normas. Como $x$ e $m$ são arbitrários concluímos que $P_{m}$ é contínua para todo $m \in \mathbb{N}$.

Dessa demonstração também podemos concluir que para todo $m \in \mathbb{N},\left\|P_{m}\right\| \leq c$.

Definição 1.4.6. Seja $X$ um espaço de Banach com base de Schauder $\left(x_{n}\right)$. O número $K=\sup _{m \in \mathbb{N}}\left\|P_{m}\right\|$ é a constante básica associada a base de Schauder $\left(x_{n}\right)$. Se $K=1$ diremos que a base de Schauder $\left(x_{n}\right)$ é monótona.

Observação: Levando em conta que as projeções naturais são contínuas, segue da definição 1.4.6, para todo $x \in X$, que

$$
\left\|P_{m}(x)\right\| \leq\left\|P_{m}\right\|\|x\| \leq \sup _{m \in \mathbb{N}}\left\|P_{m}\right\|\|x\|=K\|x\|
$$

Podemos dizer ainda que $K \geq 1$. A próxima demonstração nos mostrará o porquê.

Proposição 1.4.7. Se X é um espaço de Banach com base de Schauder $\left(x_{n}\right)$, então a constante básica $K \geq 1$.

Demonstração. Para todo $n \in \mathbb{N}$ temos:

$$
\left\|P_{n}\right\|=\sup _{\|x\|=1}\left\|P_{n}(x)\right\| \geq\left\|P_{n}\left(\frac{x_{n}}{\left\|x_{n}\right\|}\right)\right\|=\frac{1}{\left\|x_{n}\right\|}\left\|P_{n}\left(x_{n}\right)\right\|=\frac{\left\|x_{n}\right\|}{\left\|x_{n}\right\|}=1 .
$$


$\operatorname{Logo}, K=\sup _{n \in \mathbb{N}}\left\|P_{n}\right\| \geq 1$.

A outra desigualdade não acontece sempre. Mas, na necessidade de uma Base de Schauder monótona, a próxima proposição nos mostra que podemos renormar o espaço para a norma $\|\cdot\|_{\left(x_{n}\right)}$ e obter uma base de Schauder monótona.

Proposição 1.4.8. Se X é um espaço de Banach com base de Schauder $\left(x_{n}\right)$, então $\left(x_{n}\right)$ é base de Schauder monótona em $\left(X,\|\cdot\|_{\left(x_{n}\right)}\right)$.

Demonstração. Para todo $x \in X$, temos que existe uma única sequência de escalares $\left(a_{n}\right)$ tal que $\left\|x-\sum_{n=1}^{k} a_{n} x_{n}\right\| \rightarrow 0$, quando $k \rightarrow \infty$. Como visto na Proposição 1.4.3, as duas normas são equivalentes em $X$, logo:

$$
\left\|x-\sum_{n=1}^{k} a_{n} x_{n}\right\|_{\left(x_{n}\right)} \leq c\left\|x-\sum_{n=1}^{k} a_{n} x_{n}\right\| \rightarrow 0,
$$

em que $c \in \mathbb{R}$ é uma constante proveniente da equivalência das normas. Portanto $\left\|x-\sum_{n=1}^{k} a_{n} x_{n}\right\|_{\left(x_{n}\right)} \rightarrow 0$ quando $k \rightarrow \infty$. Novamente pela equivalência das normas, se existirem duas sequências de escalares $\left(a_{n}\right)$ e $\left(b_{n}\right)$ tais que $\left\|\sum_{n=1}^{k}\left(a_{n}-b_{n}\right) x_{n}\right\|_{\left(x_{n}\right)} \rightarrow 0$, então $\left\|\sum_{n=1}^{k}\left(a_{n}-b_{n}\right) x_{n}\right\|$ também irá para zero e, por $\left(x_{n}\right)$ ser base de Schauder em $X$ com a norma original temos, para todo $n \in \mathbb{N}, a_{n}=b_{n}$. Mostramos então que para cada $x \in\left(X,\|\cdot\|_{\left(x_{n}\right)}\right)$ existe uma única sequência de escalares $\left(a_{n}\right)$ tal que $x=\sum_{n=1}^{k} a_{n} x_{n}$, isto é, $\left(x_{n}\right)$ é base de Schauder para $\left(X,\|\cdot\|_{\left(x_{n}\right)}\right)$.

Denotaremos a constante básica associada a base $\left(x_{n}\right)$ no espaço $\left(X,\|\cdot\|_{\left(x_{n}\right)}\right)$ por $K^{\prime}$. Para vermos que essa base é monótona, ou seja $K^{\prime}=1$, observemos que, por $\left(X,\|\cdot\|_{\left(x_{n}\right)}\right)$ ser espaço de Banach com base de Schauder, pela Proposição 1.4.7 temos $K^{\prime} \geq 1$. Para a outra desigualdade notemos, para todo $m \in \mathbb{N}$ e $x=\sum_{n=1}^{\infty} a_{n} x_{n} \in X$, a seguinte inequação:

$$
\left\|P_{m}(x)\right\|_{\left(x_{n}\right)}=\left\|\sum_{n=1}^{m} a_{n} x_{n}\right\|_{\left(x_{n}\right)}=\sup _{k \leq m}\left\|\sum_{n=1}^{k} a_{n} x_{n}\right\| \leq \sup _{k \in \mathbb{N}}\left\|\sum_{n=1}^{k} a_{n} x_{n}\right\|=\|x\|_{\left(x_{n}\right)} .
$$

Assim, para todo $m \in \mathbb{N},\left\|P_{m}\right\|_{\left(x_{n}\right)} \leq 1$ e portanto $K^{\prime}=\sup _{n \in \mathbb{N}}\left\|P_{n}\right\|_{\left(x_{n}\right)} \leq 1 . \operatorname{Logo}\left(x_{n}\right)$ é base monótona de $\left(X,\|\cdot\|_{\left(x_{n}\right)}\right)$.

A constante básica também nos trará mais uma majoração para a norma das somas parciais, fornecendo mais uma ferramenta para trabalharmos nos capítulos adiante. 
Proposição 1.4.9. Se $\left(x_{n}\right)$ é uma base de Schauder para um espaço de Banach $X$ e K a sua constante básica, então para todo $x \in X$ existe uma única sequência de escalares $\left(a_{n}\right)$ tal que $x=\sum_{n=1}^{\infty} a_{n} x_{n}$ e vale a desigualdade

$$
\left\|\sum_{n=1}^{m} a_{n} x_{n}\right\| \leq K\left\|\sum_{n=1}^{\infty} a_{n} x_{n}\right\|,
$$

para todo $m \in \mathbb{N}$. Ainda, se $m_{1}, m_{2} \in \mathbb{N}$, com $m_{1}<m_{2}$ então para todo $x=\sum_{n=1}^{\infty} a_{n} x_{n} \in X$, com cada $a_{n}$ real, também temos:

$$
\left\|\sum_{n=1}^{m_{1}} a_{n} x_{n}\right\| \leq K\left\|\sum_{n=1}^{m_{2}} a_{n} x_{n}\right\| .
$$

Demonstração. Para a primeira desigualdade, observamos que:

$$
\begin{aligned}
\left\|\sum_{n=1}^{m} a_{n} x_{n}\right\| & =\left\|P_{m}\left(\sum_{n=1}^{\infty} a_{n} x_{n}\right)\right\| \\
& \leq\left\|P_{m}\right\|\left\|\sum_{n=1}^{\infty} a_{n} x_{n}\right\| \\
& \leq \sup _{m \in \mathbb{N}}\left\|P_{m}\right\|\left\|\sum_{n=1}^{\infty} a_{n} x_{n}\right\| \\
& =K\left\|\sum_{n=1}^{\infty} a_{n} x_{n}\right\| .
\end{aligned}
$$

Já para obtermos a segunda desigualdade, notemos que $P_{m_{1}}(x)=P_{m_{1}}\left(P_{m_{2}}(x)\right)$ para todo $x \in X$ e assim,

$$
\begin{aligned}
\left\|\sum_{n=1}^{m_{1}} a_{n} x_{n}\right\| & =\left\|P_{m_{1}}\left(\sum_{n=1}^{\infty} a_{n} x_{n}\right)\right\| \\
& =\left\|P_{m_{1}}\left(P_{m_{2}}\left(\sum_{n=1}^{\infty} a_{n} x_{n}\right)\right)\right\| \\
& =\left\|P_{m_{1}}\left(\sum_{n=1}^{m_{2}} a_{n} x_{n}\right)\right\| \\
& \leq\left\|P_{m_{1}}\right\|\left\|\sum_{n=1}^{m_{2}} a_{n} x_{n}\right\| \\
& \leq \sup _{m \in \mathbb{N}}\left\|P_{m}\right\|\left\|\sum_{n=1}^{m_{2}} a_{n} x_{n}\right\|
\end{aligned}
$$




$$
=K\left\|\sum_{n=1}^{m_{2}} a_{n} x_{n}\right\| .
$$

Esse resultado nos possibilita caracterizar o comportamento das projeções naturais na bola fechada de um espaço de Banach. Como veremos na próxima proposição.

Proposição 1.4.10. Se X é um espaço de Banach com base de Schauder monótona $\left(x_{n}\right)$, então $P_{m}\left(B_{X}\right)=B_{\mathrm{span}\left\{x_{1}, \ldots, x_{m}\right\}}, \operatorname{com} m \in \mathbb{N}$.

Demonstração. Tome $x=\sum_{n=1}^{\infty} \alpha_{n} x_{n} \in B_{X}$ qualquer, então pela Proposição 1.4.9:

$$
\left\|P_{m}(x)\right\|=\left\|\sum_{n=1}^{m} \alpha_{n} x_{n}\right\| \leq K\left\|\sum_{n=1}^{\infty} \alpha_{n} x_{n}\right\| \leq 1
$$

Daí concluímos que $P_{m}(x) \in B_{\text {span }\left\{x_{1}, \ldots, x_{m}\right\}}$ e, pela arbitrariedade de $x, P_{m}\left(B_{X}\right) \subset B_{\text {span }\left\{x_{1}, \ldots, x_{m}\right\}}$.

Agora, para demonstrar a outra inclusão tome $y \in B_{\text {span }\left\{x_{1}, \ldots, x_{m}\right\}}$. Como $P_{m}(y)=y$ e $\|y\| \leq 1$, temos que $y \in P_{m}\left(B_{X}\right)$ e, pela arbitrariedade de $y, P_{m}\left(B_{X}\right) \supset B_{\operatorname{span}\left\{x_{1}, \ldots, x_{m}\right\}}$.

Antes de prosseguirmos, vamos ver um teorema que nos fornecerá mais uma caracterização para bases de Schauder e que será uma ferramenta muito utilizada nas próximas demonstrações.

Teorema 1.4.11. Uma sequência $\left(x_{n}\right)$ num espaço de Banach $X$ é uma base de Schauder para $X$ se, e somente se:

(i) Para todo $n \in \mathbb{N}, x_{n} \neq 0$;

(ii) Existe $M \in \mathbb{R}$ tal que:

$$
\left\|\sum_{n=1}^{m_{1}} \alpha_{n} x_{n}\right\| \leq M\left\|\sum_{n=1}^{m_{2}} \alpha_{n} x_{n}\right\|,
$$

em que $m_{1}, m_{2} \in \mathbb{N}, m_{1} \leq m_{2}, \sum_{n=1}^{m_{2}} \alpha_{n} x_{n} \in X$ e $\alpha_{1}, \ldots, \alpha_{m_{2}} \in \mathbb{K}$;

(iii) $\overline{\operatorname{span}\left\{x_{n}: n \in \mathbb{N}\right\}}=X$.

Demonstração. $(\Rightarrow)$ A definição de base de Schauder acarreta (i) e (iii), enquanto (ii) é resultado da Proposição 1.4.9.

$(\Leftarrow)$ Tome uma sequência $\left(x_{n}\right) \subset X$ que satisfaça (i), (ii) e (iii). Suponha que existam duas sequências de escalares $\left(\beta_{n}\right)$ e $\left(\gamma_{n}\right)$ tais que as séries $\sum_{n=1}^{\infty} \beta_{n} x_{n}$ e $\sum_{n=1}^{\infty} \gamma_{n} x_{n}$ convirjam para um mesmo ponto em $X$. Por (ii), existe $M>0$ tal que:

$$
\left|\beta_{1}-\gamma_{1}\right|\left\|x_{1}\right\| \leq M\left\|\sum_{n=1}^{\infty}\left(\beta_{n}-\gamma_{n}\right) x_{n}\right\|
$$




$$
\begin{aligned}
& =M\left\|\sum_{n=1}^{\infty} \beta_{n} x_{n}-\sum_{n=1}^{\infty} \gamma_{n} x_{n}\right\| \\
& =0 .
\end{aligned}
$$

Como $x_{1} \neq 0$, concluímos que $\beta_{1}=\gamma_{1}$. Por um argumento indutivo em $m$, suponha que para todo natural $k<m, \beta_{k}=\gamma_{k}$. Então, novamente por (ii) existe $M>0$ tal que:

$$
\begin{aligned}
\left|\beta_{m}-\gamma_{m}\right|\left\|x_{m}\right\| & \leq M\left\|\sum_{n=1}^{m}\left(\beta_{n}-\gamma_{n}\right) x_{n}\right\| \\
& \leq M\left\|\sum_{n=1}^{\infty}\left(\beta_{n}-\gamma_{n}\right) x_{n}\right\| \\
& =M\left\|\sum_{n=1}^{\infty} \beta_{n} x_{n}-\sum_{n=1}^{\infty} \gamma_{n} x_{n}\right\| \\
& =0 .
\end{aligned}
$$

Portanto $\beta_{m}=\gamma_{m}$ e, pela indução, $\beta_{n}=\gamma_{n}$ para todo $n \in \mathbb{N}$. Logo, para nenhum elemento $x \in X$ existirá mais que uma sequência $\left(\alpha_{n}\right) \subset \mathbb{K}$ tal que $x=\sum_{n=1}^{\infty} \alpha_{n} x_{n}$.

Agora veremos que para todo $x \in X$ existirá uma sequência de escalares $\left(\alpha_{n}\right)$ tal que $x=\sum_{n=1}^{\infty} \alpha_{n} x_{n}$. Para isso, para cada $m \in \mathbb{N}$, considere o operador $p_{m}: \operatorname{span}\left\{x_{n}: n \in \mathbb{N}\right\} \rightarrow$ $\operatorname{span}\left\{x_{1}, \ldots, x_{m}\right\}$ definido por:

$$
p_{m}\left(\sum_{n=1}^{\infty} a_{n} x_{n}\right)=\sum_{n=1}^{m} a_{n} x_{n}
$$

na qual $\left(a_{n}\right)$ é uma sequência em $\mathbb{K}$ com um número finito de elementos não nulos. $p_{m}$ é claramente linear e (ii) nos garante a sua continuidade e que sua norma é menor que $M$. Portanto, pelo Teorema 1.2.2, existe um único operador linear contínuo $P_{m}: X \rightarrow$ $\operatorname{span}\left\{x_{1}, \ldots, x_{m}\right\}$ tal que $P_{m}(x)=p_{m}(x)$, para todo $x \in \operatorname{span}\left\{x_{n}: n \in \mathbb{N}\right\}$ e $\left\|P_{m}\right\|=\left\|p_{m}\right\|$. Portanto sua norma também será menor que $M$. Então, para todo $x \in X, y \in \operatorname{span}\left\{x_{n}\right.$ : $n \in \mathbb{N}\}$ e $m \in \mathbb{N}:$

$$
\begin{aligned}
\left\|P_{m}(x)-x\right\| & \leq\left\|P_{m}(x)-P_{m}(y)\right\|+\left\|P_{m}(y)-y\right\|+\|y-x\| \\
& =\left\|P_{m}(x-y)\right\|+\left\|P_{m}(y)-y\right\|+\|x-y\| \\
& \leq M\|x-y\|+\left\|P_{m}(y)-y\right\|+\|x-y\| \\
& =(M+1)\|x-y\|+\left\|p_{m}(y)-y\right\| .
\end{aligned}
$$


Fazendo $m \rightarrow \infty$ em (1.6), segue que

$$
\begin{array}{r}
\lim _{m \rightarrow \infty}\left\|P_{m}(x)-x\right\| \leq \lim _{m \rightarrow \infty}\left((M+1)\|x-y\|+\left\|p_{m}(y)-y\right\|\right), \\
\text { para todo } x \in X \text { e } y \in \operatorname{span}\left\{x_{n}: n \in \mathbb{N}\right\} .
\end{array}
$$

Como $p_{m}(y)$ converge para $y, \lim \left\|P_{m}(y)-y\right\|=0$, e daí

$$
\lim _{m \rightarrow \infty}\left\|P_{m}(x)-x\right\| \leq(M+1)\|x-y\|, \text { para todo } x \in X \text { e } y \in \operatorname{span}\left\{x_{n}: n \in \mathbb{N}\right\}
$$

Como $\operatorname{span}\left\{x_{n}: n \in \mathbb{N}\right\}$ é denso em $X$, podemos tomar $y$ tão próximo de $x$ quanto se queira, portanto, para todo $x \in X, \lim _{m \rightarrow \infty}\left\|P_{m}(x)-x\right\|=0$, isto é, $\lim _{m \rightarrow \infty} P_{m}(x)=x$.

Sejam $j \in \mathbb{N}$ e $y \in \operatorname{span}\left\{x_{n}: n \in \mathbb{N}\right\}$. Logo existe uma sequência $\left(a_{n}\right) \in \mathbb{K}$ tal que $y=\sum_{n=1}^{\infty} a_{n} x_{n}$. Assim,

$$
P_{j+1}(y)=p_{j+1}(y)=\sum_{n=1}^{j+1} a_{n} x_{n}:=\sum_{n=1}^{\infty} b_{n} x_{n},
$$

onde $b_{1}=a_{1}, b_{2}=a_{2}, \ldots, b_{j+1}=a_{j+1}$ e $b_{j+2}=b_{j+3}=\ldots=0$. Donde

$$
P_{j}\left(P_{j+1}(y)\right)=P_{j}\left(\sum_{n=1}^{\infty} b_{n} x_{n}\right)=\sum_{n=1}^{j} b_{n} x_{n}=\sum_{n=1}^{j} a_{n} x_{n}=p_{j}(y)=P_{j}(y) .
$$

Ou seja, as funções contínuas $P_{j} \circ P_{j+1}$ e $P_{j}$ coincidem no conjunto denso $\operatorname{span}\left\{x_{n}: n \in \mathbb{N}\right\}$, e por isso coincidem em $X$.

Agora tome $x \in X$ qualquer e vamos construir uma sequência de escalares $\left(\alpha_{n}\right)$ tal que $x=\sum_{n=1}^{\infty} \alpha_{n} x_{n}$. Seja $\alpha_{1}$ o escalar tal que $P_{1}(x)=\alpha_{1} x_{1}$. Como $P_{1} P_{2}(x)=P_{1}(x)$ e $P_{2}: X \rightarrow$ $\operatorname{span}\left\{x_{1}, x_{2}\right\}$, então existe $\alpha_{2} \in \mathbb{K}$ tal que $P_{2}(x)=\alpha_{1} x_{1}+\alpha_{2} x_{2}$. Da mesma forma, por $P_{2} P_{3}(x)=P_{2}(x)$ e $P_{3}: X \rightarrow \operatorname{span}\left\{x_{1}, x_{2}, x_{3}\right\}$, existe $\alpha_{3} \in \mathbb{K}$ tal que $P_{3}(x)=\alpha_{1} x_{1}+\alpha_{2} x_{2}+$ $\alpha_{3} x_{3}$. Indutivamente, para $k \in \mathbb{N}$, se $P_{k}(x)=\sum_{n=1}^{k} \alpha_{k} x_{k}$ então, por $P_{k} P_{k+1}(x)=P_{k}(x)$ e $P_{k}$ : $X \rightarrow \operatorname{span}\left\{x_{1}, \ldots, x_{k}\right\}$, existe $\alpha_{k+1} \in \mathbb{K}$ tal que $P_{k+1}(x)=\sum_{n=1}^{k+1} \alpha_{k} x_{k}$. Portanto essa sequência $\left(\alpha_{n}\right) \subset \mathbb{K}$ é tal que, para todo $m \in \mathbb{N}, P_{m}(x)=\sum_{n=1}^{m} \alpha_{k} x_{k}$ e $x=\lim _{m \rightarrow \infty} P_{m}(x)=\sum_{n=1}^{\infty} \alpha_{k} x_{k}$. Esse argumento de existência junto ao de unicidade mostrado primeiramente justificam que a sequência $\left(x_{n}\right)$ é uma base de Schauder para $X$.

Corolário 1.4.12. Uma sequência $\left(x_{n}\right)$ num espaço de Banach $X$ é básica se, e somente se, cada $x_{n}$ é não nulo e existe um número real $M$ tal que:

$$
\left\|\sum_{n=1}^{m_{1}} \alpha_{n} x_{n}\right\| \leq M\left\|\sum_{n=1}^{m_{2}} \alpha_{n} x_{n}\right\|
$$


em que $m_{1}, m_{2} \in \mathbb{N}, m_{1} \leq m_{2}, \sum_{n=1}^{m_{2}} \alpha_{n} x_{n} \in X$ e $\alpha_{1}, \ldots, \alpha_{m_{2}} \in \mathbb{R}$

O conceito de funcionais coeficientes associados a uma base de Schauder é importante na análise funcional. Esses funcionais são definidos de modo a lembrar os elementos da base dual de um espaço vetorial de dimensão finita.

Definição 1.4.13. Seja $X$ um espaço de Banach com base de Schauder $\left(x_{n}\right)$. Para cada $k \in \mathbb{N}$ considere a função $x_{k}^{*}: X \rightarrow \mathbb{K}$ definida por:

$$
x_{k}^{*}\left(\sum_{i=1}^{\infty} \alpha_{i} x_{i}\right)=\alpha_{k} .
$$

Chamaremos essa função de $k$-ésimo funcional coeficiente associado a base $\left(x_{n}\right)$.

Agora, para $k \in \mathbb{N}$ e $x_{k} \in\left(x_{n}\right)$ considere a função $T_{k}: \operatorname{span}\left\{x_{k}\right\} \rightarrow \mathbb{K}$ definida por:

$$
T_{k}\left(\lambda x_{k}\right)=\lambda
$$

$T_{k}$ é claramente linear. Veremos então que $T_{k}$ é contínua:

$$
\left|T_{k}\left(\lambda x_{k}\right)\right|=|\lambda|=\frac{|\lambda|}{\left\|x_{k}\right\|}\left\|x_{k}\right\|=\frac{1}{\left\|x_{k}\right\|}\left\|\lambda x_{k}\right\|
$$

em que $\lambda \in \mathbb{K}$ é qualquer. Portanto $T_{k}$ é limitada e consequentemente contínua.

Proposição 1.4.14. Para cada $k \in \mathbb{N}, x_{k}^{*} \in X^{*}$.

Demonstração. Claramente $x_{k}^{*}$ é linear. Para $k \geq 2, x_{k}^{*}=T_{k} \circ\left(P_{k}-P_{k-1}\right)$ e, para $k=$ $1, x_{1}^{*}=T_{1} \circ P_{1}$. Assim, cada $x_{k}^{*}$ é uma composição de aplicações contínuas e portanto $x_{k}^{*}$ é contínua.

Aqui temos outro resultado em que os funcionais coeficientes tem um papel próximo aos elementos da base dual canônica em espaços com dimensão finita:

Proposição 1.4.15. Se $\left(x_{n}\right)$ é uma base de Schauder para um espaço de Banach X, então a sequência $\left(x_{n}^{*}\right)$ é básica em $X^{*}$.

Demonstração. Seja $K$ a constante básica de $\left(x_{n}\right)$ e considere o funcional $\sum_{n=1}^{m_{1}} \alpha_{n} x_{n}^{*}$. Como $\left\|\sum_{n=1}^{m_{1}} \alpha_{n} x_{n}^{*}\right\|=\sup _{|x|=1}\left\{\left|\sum_{n=1}^{m_{1}} \alpha_{n} x_{n}^{*}(x)\right|\right\}$ então, para um $\delta>0$ qualquer, podemos tomar um $x=\sum_{n=1}^{\infty} \beta_{n} x_{n} \in S_{X}$ tal que $\left|\sum_{n=1}^{m_{1}} \alpha_{n} x_{n}^{*}(x)\right| \geq\left\|\sum_{n=1}^{m_{1}} \alpha_{n} x_{n}^{*}\right\|-\delta$. Para um $m_{2} \geq m_{1}$ teremos:

$$
\left\|\sum_{n=1}^{m_{1}} \alpha_{n} x_{n}^{*}\right\| \leq\left|\sum_{n=1}^{m_{1}} \alpha_{n} x_{n}^{*}(x)\right|+\delta
$$




$$
\begin{aligned}
& =\left|\left(\sum_{n=1}^{m_{1}} \alpha_{n} x_{n}^{*}\right)\left(\sum_{n=1}^{\infty} \beta_{n} x_{n}\right)\right|+\delta \\
& =\left|\left(\sum_{n=1}^{m_{1}} \alpha_{n} \beta_{n}\right)\right|+\delta \\
& =\left|\left(\sum_{n=1}^{m_{2}} \alpha_{n} x_{n}^{*}\right)\left(\sum_{n=1}^{m_{1}} \beta_{n} x_{n}\right)\right|+\delta \\
& \leq\left\|\sum_{n=1}^{m_{1}} \beta_{n} x_{n}\right\|\left\|\sum_{n=1}^{m_{2}} \alpha_{n} x_{n}^{*}\right\|+\delta \\
& \leq K\left\|\sum_{n=1}^{\infty} \beta_{n} x_{n}\right\|\left\|\sum_{n=1}^{m_{2}} \alpha_{n} x_{n}^{*}\right\|+\delta \text { (pela Proposição 1.4.9) } \\
& =K\left\|\sum_{n=1}^{m_{2}} \alpha_{n} x_{n}^{*}\right\|+\delta .
\end{aligned}
$$

Pela arbitrariedade de $\delta$ segue que:

$$
\left\|\sum_{n=1}^{m_{1}} \alpha_{n} x_{n}^{*}\right\| \leq K\left\|\sum_{n=1}^{m_{2}} \alpha_{n} x_{n}^{*}\right\| .
$$

Como cada $x_{n}^{*}$ é não nulo, o corolário do Teorema 1.4.11 nos garante que $\left(x_{n}^{*}\right)$ é sequência básica. 


\section{Capítulo 2}

\section{Conjuntos lineáveis}

Num espaço vetorial de dimensão infinita podemos encontrar em alguns subconjuntos estruturas de espaço vetorial. Essas estruturas nos auxiliam a mensurar o quão grande esses subconjuntos são. Dessa ideia segue a definição.

Definição. Um subconjunto $S$ de um espaço vetorial $X$ é lineável se $S \cup\{0\}$ contém um subespaço de dimensão infinita.

Alguns exemplos de conjuntos lineáveis, cujos elementos são funções patológicas, foramnos apresentados em [8]. Neste capítulo veremos alguns teoremas apresentados nesse artigo com enfoque na lineabilidade dos conjuntos de funções $C^{\infty}$ não analíticas, de funções que possuem apenas uma quantidade finita de pontos de continuidade, funções cujas derivadas são ilimitadas num intervalo fechado e funções sobrejetoras em todo lugar que se anulam quase sempre.

\subsection{Funções $C^{\infty}$ não analíticas}

Em [8] é provado que esse conjunto é algebrável, isto é, a existência de uma álgebra contida na união desse conjunto com $\{0\}$. Como nosso enfoque são conjuntos lineáveis, estudaremos esse aspecto e veremos, portanto, que o conjunto das funções $C^{\infty}$ não analíticas é lineável. Para seguirmos adiante, precisaremos de um conceito preliminar a respeito de funções analíticas. Lembremos que uma função é $C^{\infty}$ se para todo natural $n \in \mathbb{N}$ a sua derivada de $n$-ésima ordem existe e é contínua. E se $D \subset \mathbb{R}$ é um aberto e $f: D \rightarrow \mathbb{R}$ é uma função $C^{\infty}$, então $f$ é analítica se para todo ponto $x_{0} \in D$ existe um intervalo aberto $V_{x_{0}} \subset D$, com $x_{0} \in V_{x_{0}}$, tal que

$$
f(x)=\sum_{i=0}^{\infty} \frac{f^{(i)}\left(x_{0}\right)}{i !}\left(x-x_{0}\right), \text { para todo } x \in V_{x_{0}} .
$$


O próximo teorema nos mostra como se comporta uma função analítica quando todas as derivadas se anulam num ponto de seu domínio.

Teorema 2.1.1. Seja I um intervalo e $x$ um elemento de $I$. Se $f: I \rightarrow \mathbb{R}$ é analítica, $f(x)=0$ e, para todo $n \in \mathbb{N}, f^{(n)}(x)=0$, então $f$ se anula em todo $I$.

Demonstração. Essa demonstração pode ser encontrada em [15], páginas 402 e 403.

Para o próximo lema, relembremos a Regra de L'Hôpital. Sejam $f$ e $g$ funções reais deriváveis definidas num aberto $I, \operatorname{com} g^{\prime}(x) \neq 0$, para todo $x \in I$. Se

$$
\lim _{x \rightarrow p} f(x)=\lim _{x \rightarrow p} g(x)=0 \text { ou } \lim _{x \rightarrow p} f(x)=\lim _{x \rightarrow p} g(x)=\infty \text { e } \lim _{x \rightarrow p} \frac{f^{\prime}(x)}{g^{\prime}(x)} \text { existe. }
$$

Então

$$
\lim _{x \rightarrow p} \frac{f(x)}{g(x)}=\lim _{x \rightarrow p} \frac{f^{\prime}(x)}{g^{\prime}(x)}
$$

No seguinte lema estudaremos o comportamento da função $x^{-p} e^{-\beta x^{-2}}$ próximo ao zero em que $p$ é natural e $\beta$ real positivo. Essa função será utilizada na demonstração do Teorema 2.1.3.

Lema 2.1.2. Sejam $\left.f_{p}, g_{p}:\right] 0,+\infty\left[\rightarrow \mathbb{R}\right.$ definidas por $f(x)=x^{-p}$ e $g(x)=e^{\beta x^{-2}}$, para cada $p \in \mathbb{N}$ e $\beta \in] 0,+\infty[$. Então

$$
\lim _{x \rightarrow 0} \frac{f_{p}(x)}{g_{p}(x)}=\lim _{x \rightarrow 0} \frac{x^{-p}}{e^{\beta x^{-2}}}=0, \text { para todo } p \in \mathbb{N}
$$

Demonstração. $f_{p}(x)$ e $g_{p}(x)$ são deriváveis para todo $p \in \mathbb{N}$ e $g_{p}^{\prime}(x)=-2 \beta x^{-3} e^{\beta x^{-2}} \neq 0$, para todo $x \in \mathbb{R}$. Vale também

$$
\lim _{x \rightarrow 0} f_{p}(x)=\lim _{x \rightarrow 0} g_{p}(x)=\infty .
$$

Portanto, podemos aplicar a regra de L'Hôpital.

Aplicando a regra de L'Hôpital, notamos que a potência de $x$ no numerador aumenta em 2 e a fração fica multiplicada por uma constante real $c$, da seguinte forma:

$$
\lim _{x \rightarrow 0} \frac{x^{-p}}{e^{\beta x^{-2}}}=\lim _{x \rightarrow 0} \frac{-p x^{-p-1}}{-2 \beta x^{-3} e^{\beta x^{-2}}}=\lim _{x \rightarrow 0} c \frac{x^{-p+2}}{e^{\beta x^{-2}}}=c \lim _{x \rightarrow 0} \frac{x^{-p+2}}{e^{\beta x^{-2}}} .
$$

Assim, podemos aplicar a regra de L'hôpital várias vezes até conseguirmos calcular o limite. Precisaremos dividir o problema em dois casos, quando $p$ é par e quando $p$ é ímpar.

- $p=2 k$ : 
(a) Quando $p=2=2 \cdot 1$,

$$
\lim _{x \rightarrow 0} \frac{x^{-2}}{e^{\beta x^{-2}}}=\lim _{x \rightarrow 0} \frac{-2 x^{-2-1}}{-2 \beta x^{-3} e^{\beta x^{-2}}}=\lim _{x \rightarrow 0} \frac{1}{\beta} \cdot \frac{x^{0}}{e^{\beta x^{-2}}}=\frac{1}{\beta} \lim _{x \rightarrow 0} \frac{1}{e^{\beta x^{-2}}} .
$$

Observemos que a regra de L'Hôpital foi aplicada apenas uma vez.

(b) Quando $p=4=2 \cdot 2$,

$$
\lim _{x \rightarrow 0} \frac{x^{-4}}{e^{\beta x^{-2}}}=\lim _{x \rightarrow 0} \frac{-4 x^{-4-1}}{-2 \beta x^{-3} e^{\beta x^{-2}}}=\lim _{x \rightarrow 0} \frac{2}{\beta} \cdot \frac{x^{-2}}{e^{\beta x^{-2}}}=\frac{2}{\beta} \lim _{x \rightarrow 0} \frac{x^{-2}}{e^{\beta x^{-2}}} .
$$

Aqui usamos a regra de L'hôpital uma vez e chegamos num produto de uma contante pelo limite do item (a). Portanto pelo que vimos em (a),

$$
\lim _{x \rightarrow 0} \frac{x^{-4}}{e^{\beta x^{-2}}}=\frac{2}{\beta} \lim _{x \rightarrow 0} \frac{x^{-2}}{e^{\beta x^{-2}}}=\frac{2}{\beta} \cdot \frac{1}{\beta} \lim _{x \rightarrow 0} \frac{1}{e^{\beta x^{-2}}} .
$$

Totalizando dois usos da regra de L'Hôpital.

(c) Indutivamente, quando $p=2 \cdot k$, aplicaremos a regra de L'Hôpital $k$ vezes. Assim

$$
\lim _{x \rightarrow 0} \frac{x^{-2 k}}{e^{\beta x^{-2}}}=\cdots=c \lim _{x \rightarrow 0} \frac{1}{e^{\beta x^{-2}}}
$$

onde $c$ é uma constante real positiva.

Como $\lim _{x \rightarrow 0} \frac{1}{e^{\beta x^{-2}}}=0$, então $\lim _{x \rightarrow 0} \frac{x^{-p}}{e^{\beta x^{-2}}}=0$ para todo $p$ par.

- $p=2 k+1$ :

(a) Quando $p=3=2 \cdot 1+1$,

$$
\lim _{x \rightarrow 0} \frac{x^{-3}}{e^{\beta x^{-2}}}=\lim _{x \rightarrow 0} \frac{-3 x^{-3-1}}{-2 \beta x^{-3} e^{\beta x^{-2}}}=\lim _{x \rightarrow 0} \frac{3}{2 \beta} \cdot \frac{x^{1}}{e^{\beta x^{-2}}}=\frac{3}{2 \beta} \lim _{x \rightarrow 0} \frac{x}{e^{\beta x^{-2}}} .
$$

Observemos que a regra de L'Hôpital foi usada apenas uma vez.

(b) Quando $p=5=2 \cdot 2+1$,

$$
\lim _{x \rightarrow 0} \frac{x^{-5}}{e^{\beta x^{-2}}}=\lim _{x \rightarrow 0} \frac{-5 x^{-5-1}}{-2 \beta x^{-3} e^{\beta x^{-2}}}=\lim _{x \rightarrow 0} \frac{5}{2 \beta} \cdot \frac{x^{-3}}{e^{\beta x^{-2}}}=\frac{5}{2 \beta} \lim _{x \rightarrow 0} \frac{x^{-3}}{e^{\beta x^{-2}}} .
$$

Análogo ao caso par, aplicamos a regra de L'Hôpital uma vez e chagamos no produto de uma constante pelo limite do item (a). Portanto segue

$$
\lim _{x \rightarrow 0} \frac{x^{-5}}{e^{\beta x^{-2}}}=\frac{5}{2 \beta} \lim _{x \rightarrow 0} \frac{x^{-3}}{e^{\beta x^{-2}}}=\frac{5}{2 \beta} \cdot \frac{3}{2 \beta} \lim _{x \rightarrow 0} \frac{x}{e^{\beta x^{-2}}} .
$$

Totalizando dois usos da regra de L'Hôpital. 
(c) Indutivamente, quando $p=2 \cdot k+1$, aplicamos a regra de L'Hôpital $k$ vezes e obtemos

$$
\lim _{x \rightarrow 0} \frac{x^{-(2 k+1)}}{e^{\beta x^{-2}}}=\cdots=c \lim _{x \rightarrow 0} \frac{x}{e^{\beta x^{-2}}},
$$

onde $c$ é uma constante real positiva.

Como $\lim _{x \rightarrow 0} \frac{x}{e^{\beta x^{-2}}}=0$, então $\lim _{x \rightarrow 0} \frac{x^{-p}}{e^{\beta x^{-2}}}=0$ para todo $p$ ímpar.

Logo, para todo $p \in \mathbb{N}$ e $\beta \in \mathbb{R}_{+}$, temos $\lim _{x \rightarrow 0} \frac{x^{-p}}{e^{\beta x^{-2}}}=0$.

Para mostrar que o conjunto estudado é lineável tomaremos uma família de funções as quais usaremos os resultados acima para ver que são $C^{\infty}$ e não analíticas. Depois mostraremos que essa família é base para um espaço vetorial de dimensão infinita montando uma matriz de Vandermonde e utilizando, mais uma vez, o Teorema 2.1.1.

Teorema 2.1.3. O conjunto das funções $f: \mathbb{R} \rightarrow \mathbb{R}$ que são $C^{\infty}$ e não analíticas é lineável. Demonstração. Seja $M$ o conjunto das funções $C^{\infty}$ não analíticas. Considere as funções $f_{\beta}$, $\operatorname{com} \beta \in \mathbb{R}_{+}$dadas por:

$$
f_{\beta}(x)= \begin{cases}e^{-\beta x^{-2}} & \text { se } x \neq 0 \\ 0 & \text { se } x=0\end{cases}
$$

Para cada $\beta, f_{\beta} \in M$. De fato, vamos ver que $f_{\beta}^{(n)}(0)=0$ para todo $n \in \mathbb{N}$ : Façamos uma indução em $n$. Quando $n=1$ temos:

$$
f_{\beta}^{\prime}(0)=\lim _{x \rightarrow 0} \frac{f_{\beta}(x)-f_{\beta}(0)}{x-0}=\lim _{x \rightarrow 0} \frac{f_{\beta}(x)}{x}=\lim _{x \rightarrow 0} \frac{x^{-1}}{e^{\beta x^{-2}}}
$$

O Lema 2.1.2 nos garante que $f_{\beta}^{\prime}(0)=0$. Agora suponhamos que o resultado seja válido para $n$ e mostraremos então que também vale para $n+1$. Primeiramente, notemos que para cada $n \in \mathbb{N}$ :

$$
f_{\beta}^{(n)}(x)=e^{-\beta x^{-2}} \sum_{i=1}^{k_{n}} a_{n_{i}} x^{-j_{n_{i}}}
$$

em que $k_{n} \in \mathbb{N}, i \in\left\{1, \ldots, k_{n}\right\}$ e para todo $i, a_{n_{i}} \in \mathbb{Z}$ e $j_{n_{i}} \in \mathbb{N}$. Deste modo:

$$
f_{\beta}^{(n+1)}(0)=\lim _{x \rightarrow 0} \frac{f_{\beta}^{(n)}(x)}{x}=\lim _{x \rightarrow 0} \sum_{i=1}^{k_{n}} e^{-\beta x^{-2}} a_{n_{i}} x^{-\left(1+j_{n_{i}}\right)}=\sum_{i=1}^{k_{n}} \lim _{x \rightarrow 0} e^{-\beta x^{-2}} a_{n_{i}} x^{-\left(1+j_{n_{i}}\right)} .
$$

O Lema 2.1.2 garante que o limite de cada parcela da soma existe e é zero. Portanto, $f_{\beta}^{(n+1)}(0)=0$. Logo $f_{\beta}^{(n)}(0)=0$, para todo $n \in \mathbb{N}$ e como $f_{\beta}$ não é nula, o Teorema 2.1.1 aplicado no ponto $x=0$ nos garante que $f_{\beta}$ não é analítica.

Defina o seguinte espaço vetorial $V=\operatorname{span}\left\{f_{\beta}: \beta \in \mathbb{R}_{+}\right\}$.Vamos verificar que $\left\{f_{\beta}: \beta \in\right.$ $\left.\mathbb{R}_{+}\right\}$é linearmente independente. Sejam $\alpha_{1}, \ldots, \alpha_{k} \in \mathbb{R}$ tais que $\sum_{i=1}^{k} \alpha_{i} f_{\beta_{i}}=0$. Portanto, para 
$x \neq 0$

$$
0=\left(\sum_{i=1}^{k} \alpha_{i} f_{\beta_{i}}(x)\right)^{\prime}=\sum_{i=1}^{k} \alpha_{i} f_{\beta_{i}}^{\prime}(x)=\frac{2}{x^{3}} \sum_{i=1}^{k} \alpha_{i} \beta_{i} f_{\beta_{i}}(x)
$$

Logo, $G_{1}(x):=\sum_{i=1}^{k} \alpha_{i} \beta_{i}^{1} f_{\beta_{i}}(x)=0$, para todo $x \in \mathbb{R}$ (note que, pela definição das $f_{\beta_{i}}$, $\left.G_{1}(0)=0\right)$. Então $G_{1}^{\prime}=0$. Daí segue:

$$
0=G_{1}^{\prime}(x)=\frac{2}{x^{3}} \sum_{i=1}^{k} \alpha_{i} \beta_{i}^{2} f_{\beta_{i}}(x)
$$

E $G_{2}(x):=\sum_{i=1}^{k} \alpha_{i} \beta_{i}^{2} f_{\beta_{i}}(x)$, para todo $x \in \mathbb{R}$, acarreta que $G_{2}^{\prime}=0$. Continuando com esse mesmo argumento, obtemos:

$$
G_{n}:=\sum_{i=1}^{k} \alpha_{i} \beta_{i}^{n} f_{\beta_{i}}=0, \forall n \in \mathbb{N}
$$

Notemos então que, por $\lim _{x \rightarrow+\infty} f_{\beta_{i}}(x)=1$ :

$0=\lim _{x \rightarrow+\infty} G_{n}(x)=\lim _{x \rightarrow+\infty} \sum_{i=1}^{k} \alpha_{i} \beta_{i}^{n} f_{\beta_{i}}(x)=\sum_{i=1}^{k} \alpha_{i} \beta_{i}^{n} \lim _{x \rightarrow+\infty} f_{\beta_{i}}(x)=\sum_{i=1}^{k} \alpha_{i} \beta_{i}^{n}$, para cada $n \in \mathbb{N}$.

Aplicando essa última equação para $n=0, \ldots, k-1$, montamos o seguinte sistema:

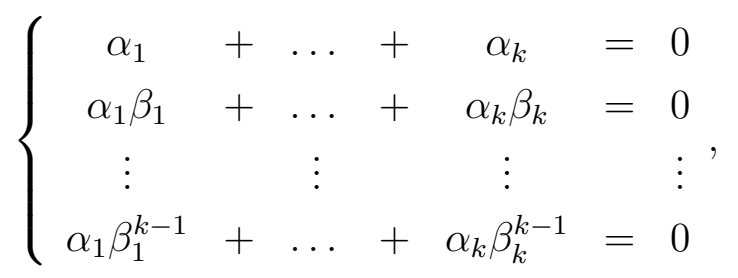

que na forma matricial fica:

$$
\left(\begin{array}{ccccc}
1 & 1 & 1 & \ldots & 1 \\
\beta_{1} & \beta_{2} & \beta_{3} & \ldots & \beta_{k} \\
\beta_{1}^{2} & \beta_{2}^{2} & \beta_{3}^{2} & \ldots & \beta_{k}^{2} \\
\vdots & \vdots & \vdots & \ddots & \vdots \\
\beta_{1}^{k-1} & \beta_{2}^{k-1} & \beta_{3}^{k-1} & \ldots & \beta_{k}^{k-1}
\end{array}\right)\left(\begin{array}{c}
\alpha_{1} \\
\alpha_{2} \\
\alpha_{3} \\
\vdots \\
\alpha_{k}
\end{array}\right)=\left(\begin{array}{c}
0 \\
0 \\
0 \\
\vdots \\
0
\end{array}\right)
$$

A matriz acima é de Vandermonde e, portanto, seu determinante é $\prod_{1 \leq i<j \leq k}\left(\beta_{j}-\beta_{i}\right)$ que é não nulo pois os $\beta_{i}$ são distintos. Concluímos que essa matriz é invertível e então a única solução para o sistema é a trivial, isto é, $\alpha_{1}=\cdots=\alpha_{k}=0$, como queríamos demonstrar. Logo, 
$V=\operatorname{span}\left\{f_{\beta}: \beta \in \mathbb{R}^{+}\right\}$é um espaço vetorial de dimensão $\mathfrak{c}$.

Falta ainda verificar que $V \subset M \cup\{0\}$. Seja $g \in V \backslash\{0\}$, então existem $\alpha_{1}, \ldots, \alpha_{k} \in \mathbb{R}$ não todos nulos tais que $g=\sum_{i=1}^{k} \alpha_{i} f_{\beta_{i}}$.

$$
g^{(n)}(0)=\sum_{i=1}^{k} \alpha_{i} f_{\beta_{i}}^{(n)}(0)=0, \forall n \in \mathbb{N}
$$

então novamente pelo Teorema 2.1.1 aplicado no ponto $x=0$ concluímos que $g$ não é analítica. Logo $g \in M \cup\{0\}$ e pela arbitrariedade de $g, V \subset M$, ou seja, $M$ é lineável.

\subsection{Funções com apenas uma quantidade finita de pontos de continuidade}

Nesse segundo conjunto estudado em [8], para verificarmos a sua linearidade também tomaremos uma família conveniente e veremos que ela gera um espaço de dimensão infinita, novamente, utilizando a construção de uma matriz de Vandermonde. Mas também utilizaremos um resultado interessante que relaciona os pontos de continuidade das funções geradas pela família com as raízes de um polinômio intrínseco à função.

Teorema 2.2.1. O conjunto de todas as funções sobre $\mathbb{R}$ as quais são contínuas apenas num número finito de pontos é lineável.

Demonstração. Para cada $i \in \mathbb{N}$ podemos definir uma função $f_{i}: \mathbb{R} \rightarrow \mathbb{R}$ por:

$$
f_{i}(x)= \begin{cases}x^{i} & \text { se } x \in \mathbb{Q} \\ -x^{i} & \text { se } x \in \mathbb{R} \backslash \mathbb{Q} .\end{cases}
$$

Agora definimos o espaço vetorial $V:=\operatorname{span}\left\{f_{i}: i \in \mathbb{N}\right\}$. Vejamos então que o conjunto $\left\{f_{i}: i \in \mathbb{N}\right\}$ é linearmente independente:

Sejam $\alpha_{1}, \ldots, \alpha_{k} \in \mathbb{R}$ tais que $\sum_{j=1}^{k} \alpha_{j} f_{i_{j}}=0$, onde $i_{1}>\cdots>i_{k}$. Ao aplicarmos a função resultante dessa soma em $2^{0}, 2^{1}, \ldots, 2^{k-1}$ obtemos o seguinte sistema:

$$
\left\{\begin{array}{ccccccc}
\alpha_{1} f_{i_{1}}\left(2^{0}\right) & + & \ldots & + & \alpha_{k} f_{i_{k}}\left(2^{0}\right) & = & 0 \\
\alpha_{1} f_{i_{1}}\left(2^{1}\right) & + & \ldots & + & \alpha_{k} f_{i_{k}}\left(2^{1}\right) & = & 0 \\
\vdots & & \vdots & & \vdots & & \vdots \\
\alpha_{1} f_{i_{1}}\left(2^{k-1}\right) & + & \ldots & + & \alpha_{k} f_{i_{k}}\left(2^{k-1}\right) & & 0
\end{array}\right.
$$


que na forma matricial fica:

$$
\left(\begin{array}{ccccc}
1 & 1 & 1 & \ldots & 1 \\
2^{i_{1}} & 2^{i_{2}} & 2^{i_{3}} & \ldots & 2^{i_{k}} \\
\left(2^{i_{1}}\right)^{2} & \left(2^{i_{2}}\right)^{2} & \left(2^{i_{3}}\right)^{2} & \ldots & \left(2^{i_{k}}\right)^{2} \\
\vdots & \vdots & \vdots & \ddots & \vdots \\
\left(2^{i_{1}}\right)^{k-1} & \left(2^{i_{2}}\right)^{k-1} & \left(2^{i_{3}}\right)^{k-1} & \ldots & \left(2^{i_{k}}\right)^{k-1}
\end{array}\right)\left(\begin{array}{c}
\alpha_{1} \\
\alpha_{2} \\
\alpha_{3} \\
\vdots \\
\alpha_{k}
\end{array}\right)=\left(\begin{array}{c}
0 \\
0 \\
0 \\
\vdots \\
0
\end{array}\right)
$$

Novamente temos que a matriz acima é de Vandermonde e seu determinante é $\prod_{1 \leq n<m \leq k}\left(2^{i_{m}}-\right.$ $\left.2^{i_{n}}\right)$ e, portanto, não nulo já que $i_{1}>\cdots>i_{k}$. Logo a matriz acima é invertível e a única solução para o sistema é $\alpha_{1}=\cdots=\alpha_{k}=0$. Assim, $\left\{f_{i}: i \in \mathbb{N}\right\}$ é uma base para $V$ que, portanto, tem dimensão $\aleph_{0}$.

Verificaremos agora que os elementos de $V \backslash\{0\}$ possuem uma quantidade finita de pontos de continuidade. Tome $F=\sum_{i=1}^{n} \alpha_{i} f_{i}$ um elemento qualquer de $V \backslash\{0\}$, onde $n \in \mathbb{N},\left\{\alpha_{j}\right\}_{i=1}^{n} \subset \mathbb{R}$ com os $\alpha_{i}$ não todos nulos. Logo, para todo $x \in \mathbb{R}$ temos que:

$$
F(x)= \begin{cases}\alpha_{1} x+\cdots+\alpha_{n} x^{n} & \text { se } x \in \mathbb{Q}, \\ -\left(\alpha_{1} x+\cdots+\alpha_{n} x^{n}\right) & \text { se } x \in \mathbb{R} \backslash \mathbb{Q} .\end{cases}
$$

Afirmo que $F$ é contínua em $x$ se, e somente se, $\alpha_{1} x+\cdots+\alpha_{n} x^{n}=0$, ou seja, se $x$ é raiz do polinômio $p(x)=\alpha_{1} x+\cdots+\alpha_{n} x^{n}$. De fato, se $F$ é contínua em $x$, como $\mathbb{Q}$ e $(\mathbb{R} \backslash \mathbb{Q})$ são densos em $\mathbb{R}$, existem sequências $\left(q_{j}\right)_{j=1}^{\infty} \subset \mathbb{Q}$ e $\left(r_{j}\right)_{j=1}^{\infty} \subset \mathbb{R} \backslash \mathbb{Q}$ que convergem para $x$. Mas para cada $j \in \mathbb{N}$, como a soma e o produto em $\mathbb{R}$ são funções contínuas,

$$
\begin{gathered}
F\left(q_{j}\right)=\alpha_{1} q_{j}+\cdots+\alpha_{n} q_{j}^{n} \underset{j \rightarrow \infty}{\longrightarrow} \alpha_{1} x+\cdots+\alpha_{n} x^{n}, \\
F\left(r_{j}\right)=-\left(\alpha_{1} r_{j}+\cdots+\alpha_{n} r_{j}^{n}\right) \underset{j \rightarrow \infty}{\longrightarrow}-\left(\alpha_{1} x+\cdots+\alpha_{n} x^{n}\right) .
\end{gathered}
$$

Da continuidade de $F$ em $x, \lim F\left(q_{j}\right)=F(x)=\lim F\left(r_{j}\right)$ e, portanto, $\alpha_{1} x+\cdots+\alpha_{n} x^{n}=0$. Por outro lado, se $\alpha_{1} x+\cdots+\alpha_{n} x^{n}=0$, tomamos uma sequência $\left(x_{j}\right)_{j=1}^{\infty} \subset \mathbb{R}$ que convirja para $x$. Assim, pela continuidade da soma e do produto em $\mathbb{R}$ :

$$
\left(F\left(x_{j}\right)\right)^{2}=\left(\alpha_{1} x_{j}+\ldots+\alpha_{n} x_{j}^{n}\right)^{2} \underset{j \rightarrow \infty}{\longrightarrow}\left(\alpha_{1} x+\ldots+\alpha_{n} x^{n}\right)^{2}=0
$$

e portanto $F\left(x_{n}\right) \underset{j \rightarrow \infty}{\longrightarrow} 0=F(x)$. Logo, $F$ é contínua em $x$.

Agora, como o polinômio $p(x)=\alpha_{1} x+\ldots+\alpha_{n} x^{n}$ tem no máximo $n$ raízes, pela afirmação acima concluímos que $F$ possui no máximo $n$ pontos de continuidade. Logo o conjunto das funções com um número finito de pontos de continuidade unido com $\{0\}$ contém o espaço 
vetorial $V$ (cuja dimensão é $\aleph_{0}$ ) e, portanto, é lineável.

\subsection{Funções cujas derivadas são ilimitadas num intervalo fechado}

Nessa seção veremos a lineabilidade do conjunto de mais uma patologia. Neste caso, a família de funções é escolhida de modo que o comportamento cíclico do seno e cosseno é usado para mostrar a independência linear dessa família tanto quanto para mostrar que a derivada das funções geradas pela família são ilimitadas no intervalo ] - 1, 1[.

Teorema 2.3.1. O conjunto das funções sobre $\mathbb{R}$ cuja derivada é ilimitada em [-1,1] é lineável.

Demonstração. Seja $P$ o conjunto de todos os números primos, então para cada $p \in P$ defina a função $f_{p}: \mathbb{R} \rightarrow \mathbb{R}$ por:

$$
f_{p}(x)= \begin{cases}x^{2} \operatorname{sen}\left(\frac{1}{p x^{2}}\right) & \text { se } x \neq 0 \\ 0 & \text { se } x=0\end{cases}
$$

As derivadas dessas funções são:

$$
f_{p}^{\prime}(x)= \begin{cases}2 x \operatorname{sen}\left(\frac{1}{p x^{2}}\right)-\left(\frac{2}{p x}\right) \cos \left(\frac{1}{p x^{2}}\right) & \text { se } x \neq 0 \\ 0 & \text { se } x=0\end{cases}
$$

que são claramente ilimitadas em $[-1,1]$ para todo $p \in P$.

Defina o espaço vetorial $V:=\operatorname{span}\left\{f_{p}: p \in P\right\}$. Temos que mostrar que as $f_{p}$ são linearmente independentes. Sejam $\alpha_{1}, \ldots, \alpha_{k} \in \mathbb{R}$ tais que $\sum_{i=1}^{k} \alpha_{i} f_{p_{i}}=0$ e $p_{1}, \ldots, p_{k} \in P$ dois a dois distintos. Então aplicando essa soma em $x_{j}:=\left(\frac{1}{p_{1 \ldots p_{j-1} p_{j+1} \ldots p_{k} \pi}}\right)^{\frac{1}{2}}$ para cada $j=1, \ldots, k$ temos:

$$
\begin{aligned}
0 & =\sum_{i=1}^{k} \alpha_{i} f_{p_{i}}\left(x_{j}\right) \\
& =\sum_{i=1}^{k} \alpha_{i} x_{j}^{2} \operatorname{sen}\left(\frac{p_{1} \ldots p_{j-1} p_{j+1} \ldots p_{k}}{p_{i}} \pi\right) \\
& =\alpha_{j} x_{j}^{2} \operatorname{sen}\left(\frac{p_{1} \ldots p_{j-1} p_{j+1} \ldots p_{k}}{p_{j}} \pi\right) \\
& :=\alpha_{j} x_{j}^{2} \operatorname{sen}\left(q_{j} \pi\right) .
\end{aligned}
$$

Como cada $q_{j} \in \mathbb{Q} \backslash \mathbb{Z}$, concluímos que $\alpha_{j}=0$ para todo $j=1, \ldots, k$. Então $\left\{f_{p}: p \in P\right\}$ é 
linearmente independente e $\operatorname{dim}(V)=\aleph_{0}$.

Para $F \in V \backslash\{0\}$ qualquer e $x \neq 0$, temos:

$$
\begin{aligned}
F^{\prime}(x) & =\left(\sum_{i=1}^{k} \alpha_{i} f_{p_{i}}\right)^{\prime}(x) \\
& =\sum_{i=1}^{k} \alpha_{i} f_{p_{i}}^{\prime}(x) \\
& =\sum_{i=1}^{k} \alpha_{i}\left(2 x \operatorname{sen}\left(\frac{1}{p_{i} x^{2}}\right)-\frac{2}{p_{i} x} \cos \left(\frac{1}{p_{i} x^{2}}\right)\right)
\end{aligned}
$$

Como $F$ não é nulo existe $l \in\{1, \ldots, k\}$ tal que $\alpha_{l} \neq 0$. Seja $\tilde{p}=p_{1} \ldots p_{l-1} p_{l+1} \ldots p_{k}$ e defina a sequência $\left(x_{j}\right)_{j=1}^{\infty} \subset \mathbb{R}$ por $x_{j}=\left(\frac{2}{(2 j+1) \tilde{p} \pi}\right)^{\frac{1}{2}}$. Ao aplicarmos a derivada de $F$ em cada elemento dessa sequência, obtemos:

$$
F^{\prime}\left(x_{j}\right)=\left(\sum_{i=1}^{k} \alpha_{i} 2 x_{j} \operatorname{sen}\left(\frac{1}{p_{i} x_{j}^{2}}\right)\right)-\alpha_{l} \frac{2}{p_{l} x_{j}} \cos \left(\frac{(2 j+1) \tilde{p} \pi}{2 p_{l}}\right) .
$$

Notemos que para a subsequência $j_{k}=2 k p_{l}$, com $k \in \mathbb{N}$, temos:

$$
\cos \left(\frac{\left(2 j_{k}+1\right) \tilde{p} \pi}{2 p_{l}}\right)=\cos \left(\frac{\tilde{p} \pi}{2 p_{l}}+k \tilde{p} 2 \pi\right)=\cos \left(\frac{\tilde{p} \pi}{2 p_{l}}\right)
$$

que é constante e não nula. Portanto quando $k \rightarrow \infty$, consequentemente $j \rightarrow \infty, x_{j} \rightarrow 0$ e $F^{\prime}\left(x_{j}\right) \rightarrow \infty$. Logo $F^{\prime}$ não é limitada em $[-1,1]$.

Pela arbitrariedade de $F$ concluímos que o conjunto das funções de derivada ilimitada em [-1,1] é lineável.

\subsection{Funções sobrejetoras em todo lugar que se anulam quase sempre}

Dizemos que uma função $f: \mathbb{R} \rightarrow \mathbb{R}$ é sobrejetora em todo lugar se para todo intervalo $(a, b)$ não trivial, $f((a, b))=\mathbb{R}$. A existência de tais funções foi notada por Lebesgue em 1904, e então mais recentemente foi demonstrado em [2] que o conjunto das funções sobrejetoras em todo lugar é lineável. Nesta seção estudaremos essas funções com a característica adicional de quando se anulam quase sempre em $\mathbb{R}$. Dizemos que uma função $f: \mathbb{R} \rightarrow \mathbb{R}$ se anula quase sempre em $\mathbb{R}$ se o conjunto $\{x \in \mathbb{R}: f(x) \neq 0\}$ tem medida nula. Um exemplo de tal função. é encontrado em [10] nas páginas 104 e 105. É recomendável que os conceitos do conjunto de Cantor e de medida sejam familiares ao leitor para esse exemplo. No fim dessa seção veremos um resultado o qual o conjunto de funções injetoras não possui o comportamento 
de lineabilidade, e mais, os espaços vetoriais contidos nesse conjunto possuem dimensão 1, contrastando a diferença de comportamento entre esse conjunto e o conjunto das funções sobrejetoras.

Para mostrar a lineabilidade do conjunto das funções sobrejetoras em todo lugar que se anulam quase sempre, tomaremos uma função qualquer desse conjunto e consideraremos a família das potências ímpares dessa função. Então usaremos a sobrejetividade dessa função para mostrar a independência linear dessa família e também, junto ao argumento de que polinômios de grau ímpar sempre admitem uma raiz real, que os elementos gerados por esta são sobrejetores em todo lugar e nulos quase sempre.

Teorema 2.4.1. O conjunto das funções sobrejetoras em todo lugar que se anulam quase sempre é lineável.

Demonstração. Seja $M$ o conjunto das funções sobrejetoras em todo lugar que se anulam quase sempre. Tome $f \in M$ fixo e considere o espaço vetorial $V:=\operatorname{span}\left\{f^{2 k+1}: k \in \mathbb{N}\right\}$.

Sejam $\alpha_{1}, \ldots, \alpha_{k} \in \mathbb{R}$ tais que $\sum_{i=1}^{k} \alpha_{i} f^{i}=0$. Por $f$ ser sobrejetora existe $r \in \mathbb{R}$ tal que $f(r)=y$. Então, $0=\sum_{i=1}^{k} \alpha_{i} f^{i}(r)=\sum_{i=1}^{k} \alpha_{i} y^{i}$. Pela arbitrariedade de $y$, o polinômio $\sum_{i=1}^{k} \alpha_{i} x^{i}=0$ para todo $x \in \mathbb{R}$, portanto, $\alpha_{1}=\ldots=\alpha_{k}=0$. Concluímos que o conjunto $\left\{f^{k}: k \in \mathbb{N}\right\}$ é linearmente independente. Como $\left\{f^{2 k+1}: k \in \mathbb{N}\right\} \subset\left\{f^{k}: k \in \mathbb{N}\right\}$, então $\left\{f^{2 k+1}: k \in \mathbb{N}\right\}$ também é linearmente independente e $\operatorname{dim}(V)=\aleph_{0}$.

Falta verificarmos que $V \subset M \cup\{0\}$. Seja $F \in V$, se $F=0$ trivialmente $F \in M \cup\{0\}$. Tomemos então $F \neq 0$ em $V$. Assim existem $\alpha_{1}=\ldots=\alpha_{k} \in \mathbb{R}$ não todos nulos tais que

$$
F=\sum_{j=1}^{k} \alpha_{j} f^{2 i_{j}+1}, \text { onde } 1 \leq i_{1}<\ldots<i_{k}
$$

Começaremos mostrando que $F$ é sobrejetora em todo lugar. Tome $s \in \mathbb{R}$ fixo, e considere a equação:

$$
\sum_{j=1}^{k} \alpha_{j} x^{2 i_{j}+1}=s .
$$

Como $2 i_{j}+1$ é ímpar, então a equação acima possui pelo menos uma solução real. Denotemos tal solução por $\gamma$. Como $f \in M$, para todo intervalo $(a, b) \subset \mathbb{R}$, existe $\xi \in(a, b)$ tal que $f(\xi)=\gamma$. De modo que

$$
F(\xi)=\sum_{j=1}^{k} \alpha_{j} f^{2 i_{j}+1}(\xi)=\sum_{j=1}^{k} \alpha_{j} \gamma^{2 i_{j}+1}=s .
$$

Logo, $F$ é sobrejetora em toda parte. 
Para ver que $F$ é nula quase sempre, basta notar que

$$
\{x \in \mathbb{R}: f(x)=0\} \subset\{x \in \mathbb{R}: F(x)=0\}
$$

Isto é:

$$
\{x \in \mathbb{R}: f(x) \neq 0\} \supset\{x \in \mathbb{R}: F(x) \neq 0\} .
$$

Como $\{x \in \mathbb{R}: f(x) \neq 0\}$ tem medida nula, então $\{x \in \mathbb{R}: F(x) \neq 0\}$ também tem. Logo, $F \in M \cup\{0\}$. Pela arbitrariedade de $F, V \subset M \cup\{0\}$. Logo, $M$ é lineável.

Em contrapartida, José L. Gámez-Merino, Seoane-Sepúlveda e os outros autores mostraram em [7] que já o conjunto de funções injetoras não é lineável. Para essa demonstração veremos que se supormos a existência de, ao menos, duas funções injetoras que gerem um espaço, então é possível encontrar dois números reais em que a combinação linear dessas funções admitem o mesmo valor, contradizendo a suposição.

Teorema 2.4.2. O conjunto das funções injetoras de $\mathbb{R}$ em $\mathbb{R}$ não é lineável. E ainda, se $V$ é o espaço vetorial em que cada elemento não nulo é uma função injetora sobre $\mathbb{R}$, então $V$ tem dimensão 1.

Demonstração. Suponha por absurdo que exista um espaço vetorial $V$ de funções injetoras sobre $\mathbb{R}$ (a menos da nula) com dimensão 2 gerado pelo conjunto $\{f, g\} \subset V$ linearmente independente. Então tome $x, y \in \mathbb{R}$ distintos e seja $\alpha=\frac{f(x)-f(y)}{g(y)-g(x)} \in \mathbb{R}$ (observemos que $g(y)-g(x) \neq 0$ pois $g$ é injetora). Agora considere a função $h=f+\alpha g \in V \backslash\{0\}$.

$$
\begin{aligned}
h(x) & =f(x)+\frac{f(x)-f(y)}{g(y)-g(x)} g(x) \\
& =\frac{f(x)(g(y)-g(x))+g(x)(f(x)-f(y))}{g(y)-g(x)} \\
& =\frac{f(x) g(y)-f(x) g(x)+g(x) f(x)-g(x) f(y)}{g(y)-g(x)} \\
& =\frac{f(x) g(y)-g(x) f(y)}{g(y)-g(x)} \\
& =\frac{f(x) g(y)-g(x) f(y)+f(y) g(y)-f(y) g(y)}{g(y)-g(x)} \\
& =\frac{f(y)(g(y)-g(x))+g(y)(f(x)-f(y))}{g(y)-g(x)} \\
& =f(y)+\alpha g(y)=h(y) .
\end{aligned}
$$

Logo $h(x)=h(y)$, ou seja, $h$ não é injetora. Isso contraria $h$ pertencer a $V$. Logo $V$ tem dimensão 1. 


\section{Capítulo 3}

\section{Conjuntos espaçáveis}

Daremos enfoque agora a dois resultados sobre conjuntos espaçáveis apresentados por S. Głab, P.L. Kaufmann e L. Pellegrini em [11]. O primeiro resultado fala da espaçabilidade do conjunto $\mathcal{F}$ de funções de variação limitada no intervalo $[0,1]$ com um conjunto denso de descontinuidades em salto. Já o segundo resultado nos traz a espaçabilidade de funções Lebesgue integráveis em [0,1] que não são essencialmente limitadas em nenhum lugar.

A definição de conjunto espaçável remete a de conjunto lineável. Porém num conjunto espaçável temos uma estrutura topológica.

Definição. Um subconjunto $S$ de um espaço vetorial topológico $X$ é espaçável se $S \cup\{0\}$ contém um espaço vetorial fechado de dimensão infinita.

Vale destacar que nas demonstrações desses resultados são usados conceitos de bases de Schauder, diferentemente dos métodos utilizados nos teoremas vistos no capítulo anterior. Isso mostra uma abordagem mais analítica para o confronto de problemas relacionados a conjuntos lineáveis e espaçáveis.

\subsection{Funções de variação limitada com um conjunto denso de descontinuidades}

Uma função $f: \mathbb{R} \rightarrow \mathbb{R}$ possui uma descontinuidade em salto em $p \in \mathbb{R}$ se os limites laterais em $p$ são diferentes, ou seja, $\lim _{x \rightarrow p+} f(x) \neq \lim _{x \rightarrow p-} f(x)$. Denotaremos por $\mathcal{F}$ o subconjunto de $B V[0,1]$ das funções com um conjunto denso de descontinuidades em salto. No primeiro capítulo vimos alguns conceitos e resultados sobre funções de variação limitada que serão úteis para essa seção. No próximo teorema mostraremos que $\mathcal{F}$ é espaçável e, para tal, precisaremos construir uma função em específico. Para essa construção, considere a seguinte definição. 
Definição 3.1.1. Seja $A \subset \mathbb{R}$ um conjunto. A função característica de $A$, denotada por $\chi_{A}$, é definida por

$$
\chi_{A}(x):= \begin{cases}1 & \text { se } x \in A, \\ 0 & \text { caso contrário. }\end{cases}
$$

Agora, tome uma enumeração $\left\{q_{i}: i \in \mathbb{N}\right\}$ para os números racionais em ]0,1[, e considere a função:

$$
f=\sum_{i=1}^{\infty} 2^{-i} \chi_{] q_{i}, 1\right]}
$$

A função $f:[0,1] \rightarrow[0,1]$ está bem definida pois cada função característica é bem definida para todo $i \in \mathbb{N}$ e $f(x)=\sum_{i=1}^{\infty} 2^{-i} \chi_{\left.] q_{i}, 1\right]}(x) \leq \sum_{i=1}^{\infty} 2^{-i}=1$ para todo $x \in[0,1]$.

Lema 3.1.2. A função $f$ acima:

(i) É crescente;

(ii) É contínua em $[0,1] \backslash \mathbb{Q}$;

(iii) Possui saltos em cada racional em ]0,1[;

(iv) É contínua pela direita em 0 e pela esquerda em 1, isto é,

$$
\lim _{x \rightarrow 0+} f(x)=f(0) e \lim _{x \rightarrow 1-} f(x)=f(1) .
$$

Demonstração. Para cada $a \in[0,1]$ considere o conjunto $I_{a}:=\left\{i \in \mathbb{N}: q_{i}<a\right\}$. As parcelas da soma em $f$ são nulas quando $x<q_{i}$. Portanto, $f(x)=\sum_{i=1}^{\infty} 2^{-i} \chi_{\left.] q_{i}, 1\right]}(x)=\sum_{i \in I_{x}} 2^{-i}$, para todo $x \in[0,1]$.

(i) Sejam $x, y \in[0,1]$ tais que $x<y$. Notemos que $I_{x} \subset I_{y}$, assim teremos:

$$
f(y)=\sum_{i \in I_{y}} 2^{-i}=\sum_{i \in I_{x}} 2^{-i}+\sum_{i \in I_{y} \backslash I_{x}} 2^{-i}=f(x)+\sum_{i \in I_{y} \backslash I_{x}} 2^{-i} .
$$

Como as parcelas são positivas, concluímos que $f(x)<f(y)$, ou seja, $f$ é crescente.

(ii) Seja $p \in] 0,1[\backslash \mathbb{Q}$ qualquer. Então:

$$
\lim _{x \rightarrow p-} f(x)=\lim _{x \rightarrow p-} \sum_{i \in I_{x}} 2^{-i}=\sum_{i \in I_{p}} 2^{-i}=f(p) .
$$

Por outro lado:

$$
\lim _{x \rightarrow p+} f(x)=\lim _{x \rightarrow p+} \sum_{i \in I_{x}} 2^{-i}=\sum_{i \in I_{p}} 2^{-i}=f(p) .
$$

Logo $f$ é contínua em $p$ e, portanto, contínua nos irracionais dentro de $[0,1]$. 
(iii) Seja $q \in] 0,1[\cap \mathbb{Q}$. Então:

$$
\lim _{x \rightarrow q-} f(x)=\lim _{x \rightarrow q-} \sum_{i \in I_{x}} 2^{-i}=\sum_{i \in I_{q}} 2^{-i}=f(q) .
$$

Por outro lado, existe $j \in \mathbb{N}$ tal que $q_{j}=q$, portanto se $x>q$ então $j \in I_{x}$. Logo:

$$
\begin{aligned}
\lim _{x \rightarrow q+} f(x) & =\lim _{x \rightarrow q+} \sum_{i \in I_{x}} 2^{-i} \\
& =\lim _{x \rightarrow q+}\left(2^{-j}+\sum_{i \in I_{x} \backslash\{j\}} 2^{-i}\right) \\
& =2^{-j}+\sum_{i \in I_{q}} 2^{-i} \\
& =2^{-j}+f(q) .
\end{aligned}
$$

Portanto os limites laterais são diferentes, ou seja, $f$ possui um salto em $q$.

(iv)

$$
\begin{aligned}
& \text { - } \lim _{x \rightarrow 0+} f(x)=\lim _{x \rightarrow 0+} \sum_{i \in I_{x}} 2^{-i}=0=f(0) . \\
& \text { - } \lim _{x \rightarrow 1-} f(x)=\lim _{x \rightarrow 1-} \sum_{i \in I_{x}} 2^{-i}=\sum_{i \in \mathbb{N}} 2^{-i}=1=f(1) .
\end{aligned}
$$

A função $f$ definida em (3.1) pertence a $\mathcal{F}$. Pois, pelo item (i) do Lema 3.1.2, f é crescente e pela Proposição 1.3.2 a variação total de $f$ em [0,1] é calculada pela fórmula

$$
V(f)=f(1)-f(0)=\sum_{i \in I_{1}} 2^{-i}-\sum_{i \in I_{0}} 2^{-i}=1-0=1 .
$$

Logo, $f$ é de variação limitada. O item (iii) do Lema 3.1.2 garantem que $f$ possui um conjunto denso de descontinuidades em salto no intervalo $[0,1]$.

Além da função $f$ acima, também usaremos para o próximo teorema, a seguinte relação de equivalência em ]0,1[:

$$
x \sim y \Longleftrightarrow x-y \in \mathbb{Q} .
$$

Essa relação foi utilizada por Vitalli na construção de seu conhecido conjunto que serviu como contraexemplo para várias propriedades da análise, entre elas a existência de um conjunto real que não é Lebesgue mensurável. Portanto nos referiremos a ela como relação de Vitalli.

Agora temos as ferramentas necessárias para seguir para o resultado da espaçabilidade de $\mathcal{F}$. A ideia dessa demonstração é utilizar o conjunto dos representantes das classes da relação de Vitalli junto da função $f$ para montar uma família de funções que, através dos resultados vistos nessa seção e dos conceitos preliminares de bases de Schauder, gerará o 
subespaço fechado não separável de dimensão infinita buscado. A não separabilidade virá pela existência de um conjunto discreto não enumerável. Lembremos que num espaço normado, um conjunto $A$ é discreto se para todo ponto $a \in A$ existe $\epsilon(a)>0$ real, tal que $\{x \in A$ : $\|x-a\|<\epsilon(a)\}=\{a\}$.

Teorema 3.1.3. Existe um subespaço fechado não separável de $B V[0,1]$ contido em $\mathcal{F} \cup\{0\}$.

Demonstração. Seja $V$ um conjunto de representantes das classes da relação de Vitalli. Afirmo que $V$ tem cardinalidade $\mathfrak{c}$. De fato, seja $[q]=\mathbb{Q} \cap\{r \in[0,1]: r \sim q\}$ a classe que contém todos os números racionais e $\left[v_{i}\right]$ as outras classes, em que $I$ é um conjunto de índices, $i \in I$ e cada $v_{i} \in V$. Temos que $\left.[q] \cup \bigcup_{i \in I}\left[v_{i}\right]=\right] 0,1\left[\right.$ implica $|[q]|+\left|\bigcup_{i \in I}\left[v_{i}\right]\right|=\mathfrak{c}$. Supondo por absurdo que $I$ seja enumerável então, como $[q]$ e cada $\left[v_{i}\right]$ são enumeráveis e união de enumeráveis é enumerável, concluiríamos que $\aleph_{0}=\mathfrak{c}$, um absurdo. Portanto, $|I|=\mathfrak{c}$, que implica $|V|=\mathfrak{c}$.

Considere a função $f:[0,1] \rightarrow \mathbb{R}$ definida em (3.1), e para cada $v \in V$ defina $f_{v}:[0,1] \rightarrow$ $\mathbb{R}$ por:

$$
f_{v}(x):= \begin{cases}f(x-v) & \text { se } x-v \in[0,1], \quad(\text { ou seja } x \geq v), \\ f(x-v+1) & \text { caso contrário. }\end{cases}
$$

Essa função está bem definida pois $f$ está definida no intervalo $[0,1]$, e para todo $x \in[0,1]$ se $x \geq v$ então $x-v \in[0,1]$ e se $x<v$ então $x-v+1 \in[0,1]$.

Vamos estudar os limites laterais de $f_{v}$ em $v$. Sejam $t:=x-v+1$ e $u:=x-v$. Notemos que quando $x \rightarrow v-$, então $t \rightarrow 1-$; e quando $x \rightarrow v+$, então $u \rightarrow 0+$. Assim,

$$
\begin{aligned}
\lim _{x \rightarrow v-} f_{v}(x) & =\lim _{x \rightarrow v-} f(x-v+1) \\
& =\lim _{t \rightarrow 1-} f(t) \\
& =f(1) \text { (pois pelo Lema 3.1.2-(iv) } f \text { é contínua em } t=1) . \\
\lim _{x \rightarrow v+} f_{v}(x) & =\lim _{x \rightarrow v+} f(x-v) \\
& =\lim _{u \rightarrow 0+} f(u) \\
& =f(0)(\text { pois pelo Lema 3.1.2-(iv) } f \text { é contínua em } u=0) .
\end{aligned}
$$

Notemos que:

$$
\lim _{x \rightarrow v-} f_{v}(x)=1 \mathrm{e} \lim _{x \rightarrow v+} f_{v}(x)=0 .
$$

Os limites laterais das $f_{v}$ no seu respectivo ponto $v$ são diferentes. Ou seja, $f_{v}$ tem uma descontinuidade em salto em $v$. Para ter maior noção do comportamento das $f_{v}$ podemos observar a figura abaixo que é uma representação do gráfico da $f_{v}$. Note que é apenas uma representação pois não sabemos como é o crescimento das $f_{v}$ nos intervalos $[0, v[\mathrm{e}[v, 1]$. 


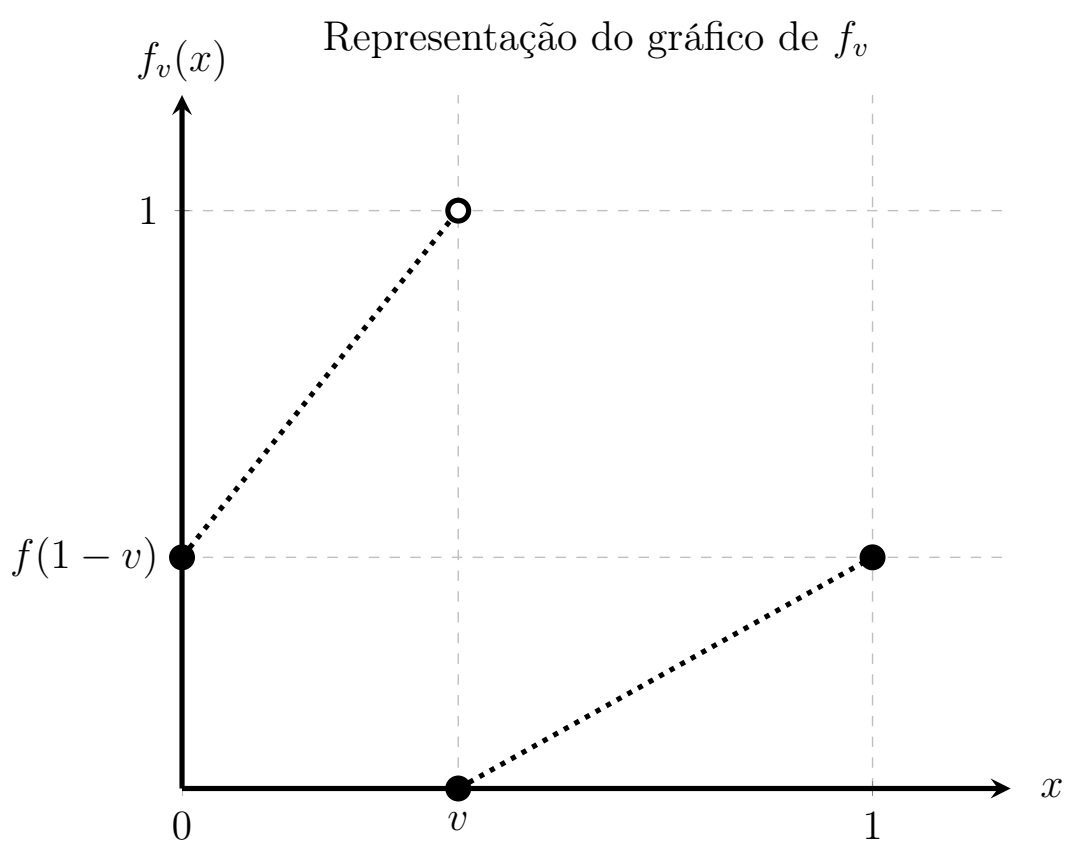

Agora, veremos como as $f_{v}$ se comportam nos outros elementos de $V$. Tome quaisquer $v, v^{\prime} \in V$ distintos, portanto, $v-v^{\prime} \notin \mathbb{Q}$. Se $v>v^{\prime}$ então existe uma vizinhança de $v$ em que os pontos são maiores que $v^{\prime}$. Assim:

$$
\begin{aligned}
\lim _{x \rightarrow v} f_{v^{\prime}}(x) & =\lim _{x \rightarrow v} f\left(x-v^{\prime}\right) \\
& =f\left(v-v^{\prime}\right)\left(\text { Pelo Lema 3.1.2, } f \text { é contínua em } v-v^{\prime}\right) \\
& =f_{v^{\prime}}(v) .
\end{aligned}
$$

Por outro lado, caso $v<v^{\prime}$, existe uma vizinhança de $v$ tal que todo ponto é menor que $v^{\prime}$. Então:

$$
\begin{aligned}
\lim _{x \rightarrow v} f_{v^{\prime}}(x) & =\lim _{x \rightarrow v} f\left(x-v^{\prime}+1\right) \\
& \left.=f\left(v-v^{\prime}+1\right) \text { (Pelo Lema 3.1.2, } f \text { é contínua em } v-v^{\prime}+1\right) \\
& =f_{v^{\prime}}(v) .
\end{aligned}
$$

Logo:

$$
\lim _{x \rightarrow v} f_{v^{\prime}}(x)=f_{v^{\prime}}(v),
$$

ou seja, $f_{v^{\prime}}$ é contínua em $v$ quando $v \neq v^{\prime}$.

O conjunto $\left\{f_{v}: v \in V\right\}$ é discreto. De fato, para $v, v^{\prime} \in V$ distintos:

$$
\begin{aligned}
\lim _{x \rightarrow v-}\left(f_{v^{\prime}}-f_{v}\right)(x) & =\lim _{x \rightarrow v-}\left(f_{v^{\prime}}\right)(x)-\lim _{x \rightarrow v-}\left(f_{v}\right)(x) \\
& =f_{v^{\prime}}(v)-1(\operatorname{por}(3.2) \mathrm{e}(3.3)) .
\end{aligned}
$$


Também temos:

$$
\begin{aligned}
\lim _{x \rightarrow v+}\left(f_{v^{\prime}}-f_{v}\right)(x) & =\lim _{x \rightarrow v+}\left(f_{v^{\prime}}\right)(x)-\lim _{x \rightarrow v+}\left(f_{v}\right)(x) \\
& =f_{v^{\prime}}(v)-0=f_{v^{\prime}}(v)(\operatorname{por}(3.2) \mathrm{e}(3.3)) .
\end{aligned}
$$

Esses resultados nos mostram que para qualquer partição de $[0,1]$ contendo $v$, a função $f_{v^{\prime}}-f_{v}$ dá um salto de tamanho 1 , de onde segue $V\left(f_{v^{\prime}}-f_{v}\right) \geq 1$ e, portanto, $\left\|f_{v^{\prime}}-f_{v}\right\| \geq$ 1. Concluímos que o conjunto $\left\{f_{v}: v \in V\right\}$ é um subconjunto discreto de $B V[0,1]$ com cardinalidade $\mathfrak{c}$.

Seja $g \in \overline{\operatorname{span}\left\{f_{v}: v \in V\right\}}$. Então há uma sequência $\left(v_{i}\right)_{i}$ de distintos elementos de $V$ tal que $g \in \overline{\operatorname{span}\left\{f_{v_{i}}: i \in \mathbb{N}\right\}}$. Nota-se que $g \in B V[0,1]$, pois as $f_{v} \in B V[0,1]$ e $B V[0,1]$ é um espaço de Banach. Para vermos que $g \in \mathcal{F}$, precisamos ver que $g$ tem um conjunto denso de saltos em $[0,1]$. E para isso, veremos primeiro que $\left(f_{v_{i}}\right)_{i}$ é sequencia básica.

Note que cada $f_{v_{i}}$ é crescente nos intervalos $\left[0, v_{i}\left[\right.\right.$ e $\left[v_{i}, 1\right]$, pois $f$ também é. Porém temos um salto decrescente em $v_{i}$. Para calcularmos a variação total de $f_{v_{i}}$ no intervalo $\left[0, v_{i}\right]$, tomemos $P\left\{x_{1}, \ldots, x_{k}\right\}$ uma partição de $\left[0, v_{i}\right]$. Assim,

$$
\begin{aligned}
\sum_{j=1}^{k-1}\left|f_{v_{i}}\left(x_{j+1}\right)-f_{v_{i}}\left(x_{j}\right)\right| & =\left(\sum_{j=1}^{k-2}\left|f_{v_{i}}\left(x_{j+1}\right)-f_{v_{i}}\left(x_{j}\right)\right|\right)+\left|f_{v_{i}}\left(x_{k}\right)-f_{v_{i}}\left(x_{k-1}\right)\right| \\
& =\left(\sum_{j=1}^{k-2}\left|f_{v_{i}}\left(x_{j+1}\right)-f_{v_{i}}\left(x_{j}\right)\right|\right)+\left|f_{v_{i}}\left(x_{k}\right)-f_{v_{i}}\left(x_{k-1}\right)\right| \\
& \leq\left(\sum_{j=1}^{k-2}\left|f_{v_{i}}\left(x_{j+1}\right)-f_{v_{i}}\left(x_{j}\right)\right|\right)+1 \\
& \leq V_{\left[0, x_{k-1}\right]}\left(f_{v_{i}}\right)+1 .
\end{aligned}
$$

$f_{v_{i}}$ é crescente no intervalo [0, $x_{k-1}$ ]. Pela Proposição 1.3.2, a variação total de $f_{v_{i}}$ em $\left[0, x_{k-1}\right]$ é dada por $V_{\left[0, x_{k-1}\right]}\left(f_{v_{i}}\right)=f_{v_{i}}\left(x_{k-1}\right)-f_{v_{i}}(0)$. Então

$$
\begin{aligned}
\sum_{j=1}^{k-1}\left|f_{v_{i}}\left(x_{j+1}\right)-f_{v_{i}}\left(x_{j}\right)\right| & \leq V_{\left[0, x_{k-1}\right]}\left(f_{v_{i}}\right)+1 \\
& =f_{v_{i}}\left(x_{k-1}\right)-f_{v_{i}}(0)+1 \\
& \leq \sup \left\{f_{v_{i}}(x): x \in\left[0, v_{i}\right]\right\}-f_{v_{i}}(0)+1 \\
& =1-f\left(1-v_{i}\right)+1 \\
& =2-f\left(1-v_{i}\right) .
\end{aligned}
$$

A partição $P$ foi tomada arbitrariamente. Logo essa desigualdade é válida para toda partição 
de $\left[0, v_{i}\right]$. Então aplicando o supremo sobre as partições de $\left[0, v_{i}\right]$ obtemos

$$
V_{\left[0, v_{i}\right]}\left(f_{v_{i}}\right)=\sup _{P} \sum_{x_{j} \in P}\left|f_{v_{i}}\left(x_{j+1}\right)-f_{v_{i}}\left(j_{i}\right)\right| \leq 2-f\left(1-v_{i}\right) .
$$

Assim, pelo item (iv) da Proposição 1.3.1, temos:

$$
\begin{aligned}
V\left(f_{v_{i}}\right) & =V_{\left[0, v_{i}\right]}\left(f_{v_{i}}\right)+V_{\left[v_{i}, 1\right]}\left(f_{v_{i}}\right) \\
& =V_{\left[0, v_{i}\right]}\left(f_{v_{i}}\right)+f_{v_{i}}(1)-f_{v_{i}}\left(v_{i}\right) \\
& \leq 2-f\left(1-v_{i}\right)+f\left(1-v_{i}\right)-0 \\
& \leq 2-f\left(1-v_{i}\right)+f\left(1-v_{i}\right) \\
& =2 .
\end{aligned}
$$

E, portanto, $\left\|f_{v_{i}}\right\|=\left|f_{v_{i}}(0)\right|+V\left(f_{v_{i}}\right) \leq 1+V\left(f_{v_{i}}\right) \leq 1+2=3$.

Agora, tome uma sequência de números reais $\left(\beta_{i}\right)_{i}$ e $n, m \in \mathbb{N}$ com $n<m$. Assim:

$$
\left\|\sum_{j=1}^{n} \beta_{j} f_{v_{j}}\right\|_{B V} \leq \sum_{j=1}^{n}\left|\beta_{j}\right|\left\|f_{v_{j}}\right\|_{B V} \leq 3 \sum_{j=1}^{n}\left|\beta_{j}\right|
$$

Por outro lado, seja $h=\sum_{j=1}^{m} \beta_{j} f_{v_{j}}$. Então, para $i=1, \ldots, m$, as equações $(3.2)$ e $(3.3)$ acarretam:

$$
\begin{aligned}
\left|\lim _{x \rightarrow v_{i}+} h(x)-\lim _{x \rightarrow v_{i}-} h(x)\right| & =\left|\lim _{x \rightarrow v_{i}+} \sum_{j=1}^{m} \beta_{j} f_{v_{j}}(x)-\lim _{x \rightarrow v_{i}-} \sum_{j=1}^{m} \beta_{j} f_{v_{j}}(x)\right| \\
& \stackrel{(3.3)}{=}\left|\sum_{j=1}^{m} \beta_{j} \lim _{x \rightarrow v_{i}+} f_{v_{j}}(x)-\sum_{j=1}^{m} \beta_{j} \lim _{x \rightarrow v_{i}-} f_{v_{j}}(x)\right| \\
& =\left|\beta_{i} \lim _{x \rightarrow v_{i}+} f_{v_{i}}(x)-\beta_{i} \lim _{x \rightarrow v_{i}-} f_{v_{i}}(x)\right| \\
& \stackrel{(3.2)}{=}\left|\beta_{i} \cdot 1-\beta_{i} \cdot 0\right| \\
& =\left|\beta_{i}-0\right| \\
& =\left|\beta_{i}\right| .
\end{aligned}
$$

Agora, seja $P=\left\{x_{1}=0, x_{2}=v_{1}-\epsilon, x_{3}=v_{1}+\epsilon, \ldots, x_{2 m}=v_{m}-\epsilon, x_{2 m+1}=v_{m}+\epsilon, x_{2 m+2}=1\right\}$ uma partição de $[0,1]$ para $\epsilon>0$. Então

$$
\sum_{i=1}^{m}\left|h\left(v_{i}+\epsilon\right)-h\left(v_{i}-\epsilon\right)\right| \leq \sum_{i=1}^{2 m+2}\left|h\left(x_{i+1}\right)-h\left(x_{i}\right)\right| \leq V(h), \text { para toda partição } P .
$$


Logo,

$$
\lim _{\epsilon \rightarrow 0} \sum_{i=1}^{m}\left|h\left(v_{i}+\epsilon\right)-h\left(v_{i}-\epsilon\right)\right| \leq V(h) .
$$

Segue,

$$
\begin{aligned}
\sum_{i=1}^{m}\left|\beta_{i}\right| & =\sum_{i=1}^{m}\left|\lim _{\epsilon \rightarrow 0} h\left(v_{i}+\epsilon\right)-\lim _{\epsilon \rightarrow 0} h\left(v_{i}-\epsilon\right)\right| \\
& =\sum_{i=1}^{m} \lim _{\epsilon \rightarrow 0}\left|h\left(v_{i}+\epsilon\right)-h\left(v_{i}-\epsilon\right)\right| \\
& =\lim _{\epsilon \rightarrow 0} \sum_{i=1}^{m}\left|h\left(v_{i}+\epsilon\right)-h\left(v_{i}-\epsilon\right)\right| \\
& \leq V(h) .
\end{aligned}
$$

E portanto,

$$
\|h\|_{B V}=|h(0)|+V(h) \geq V(h) \geq \sum_{j=1}^{m}\left|\beta_{j}\right| \geq \sum_{j=1}^{n}\left|\beta_{j}\right| .
$$

Aplicando essa desigualdade na inequação (3.4), obtemos

$$
\left\|\sum_{j=1}^{n} \beta_{j} f_{v_{j}}\right\|_{B V} \leq 3 \sum_{j=1}^{n}\left|\beta_{j}\right| \leq 3\|h\|_{B V} \leq 3\left\|\sum_{j=1}^{m} \beta_{j} f_{v_{j}}\right\|_{B V} .
$$

Pelo Corolário 1.4.12, $\left(f_{v_{i}}\right)_{i}$ é sequência básica.

Para vermos que o conjunto de saltos de $g$ é denso em $[0,1]$, considere o resultado da Proposição 1.3 .3 que implica que toda sequência convergente em $\left(B V[0,1],\|\cdot\|_{B V}\right)$ também converge em $\left(B V[0,1],\|\cdot\|_{\infty}\right)$. Portanto, essa convergência é uniforme. Como a sequência $\left(f_{v_{i}}\right)_{i}$ é básica então existe uma única sequência $\left(a_{i}\right) \subset \mathbb{R}$ tal que $g=\sum_{i=1}^{\infty} a_{i} f_{v_{i}}=\lim _{n \rightarrow \infty} \sum_{i=1}^{n} a_{i} f_{v_{i}}$. Pela uniformidade dessa convergência, temos, para cada $q \in\left[v_{1}\right]$ :

$$
\begin{aligned}
\left|\lim _{x \rightarrow q+} g(x)-\lim _{x \rightarrow q-} g(x)\right| & =\left|\lim _{x \rightarrow q+}\left(\sum_{i=1}^{\infty} a_{i} f_{v_{i}}(x)\right)-\lim _{x \rightarrow q-}\left(\sum_{i=1}^{\infty} a_{i} f_{v_{i}}(x)\right)\right| \\
& =\left|\sum_{i=1}^{\infty} a_{i} \lim _{x \rightarrow q+} f_{v_{i}}(x)-\sum_{i=1}^{\infty} a_{i} \lim _{x \rightarrow q-} f_{v_{i}}(x)\right| \\
& =\left|\sum_{i=1}^{\infty} a_{i}\left(\lim _{x \rightarrow q+} f_{v_{i}}(x)-\lim _{x \rightarrow q-} f_{v_{i}}(x)\right)\right|
\end{aligned}
$$

Para $i>1, f_{v_{i}}$ é contínua em $q$, porém pela equação $(3.2), f_{v_{1}}$ possui um salto em cada 
$q \in\left[v_{1}\right]$. Então:

$$
\begin{aligned}
\left|\lim _{x \rightarrow q+} g(x)-\lim _{x \rightarrow q-} g(x)\right| & =\left|\sum_{i=1}^{\infty} a_{i}\left(\lim _{x \rightarrow q+} f_{v_{i}}(x)-\lim _{x \rightarrow q-} f_{v_{i}}(x)\right)\right| \\
& =\left|a_{1}\left(\lim _{x \rightarrow q+} f_{v_{1}}(x)-\lim _{x \rightarrow q-} f_{v_{1}}(x)\right)\right|>0 .
\end{aligned}
$$

Segue que $g$ possui saltos em cada ponto de $\left[v_{1}\right]$, que é denso em $[0,1]$. Portanto $g \in \mathcal{F}$ e, pela arbitrariedade de $g$, concluímos que $\overline{\operatorname{span}\left\{f_{v_{i}}: i \in \mathbb{N}\right\}} \subset \mathcal{F} \cup\{0\}$.

\subsection{Funções Lebesgue integráveis não essencialmente li- mitadas em nenhum intervalo}

Essa seção é destinada ao outro resultado do artigo [11] citado no início do capítulo. Aqui veremos que o espaço das funções Lebesgue integráveis não essencialmente limitadas em nenhum intervalo é espaçável. Esse teorema generaliza um resultado de Garcia-Pacheco, Martín, e Seoane-Sepúlveda em [9] sobre o espaçabilidade do conjunto das funções Lebesgue integráveis num intervalo $I$ que não equivalem a uma função Riemann integrável em $I$. Antes de seguir para o resultado, veremos alguns conceitos necessários para a demonstração teorema. Consideraremos que o leitor já está habituado com alguns conceitos sobre medida, conjuntos mensuráveis e integrais de Lebesgue.

Primeiramente, uma função é essencialmente limitada se é limitada a menos de um conjunto de medida nula. E assim diremos que uma função não é essencialmente limitada em nenhum lugar se não é essencialmente limitada em nenhum subintervalo de seu domínio. Considere o conjunto $\mathcal{G}$ o conjunto de todas as funções Lebesgue integráveis em [0,1] que não são essencialmente limitadas em nenhum lugar.

Para vermos a espaçabilidade de $\mathcal{G}$ precisaremos trabalhar com conjuntos de Cantor generalizados. Um conjunto de Cantor generalizado $A$ num intervalo $[a, b]$, com $a, b \in \mathbb{R}$, é um subconjunto perfeito $\left(A=A^{\prime}:=\left\{x \in \mathbb{R}\right.\right.$ : existe uma sequência $\left(x_{n}\right) \subset A \backslash\{x\}$ que converge para $\mathrm{x}\}$ ), não enumerável e denso em nenhum intervalo de diâmetro $b-a$ que é obtido subtraindo de $[a, b]$ uma união enumerável de intervalos abertos de certo modo, e assim $A$ pode medir entre 0 e estritamente menos que $b-a$. Aos abertos retirados chamaremos de buracos de $A$. Mais detalhes sobre a construção desse conjunto podem ser encontrados nas páginas 88 e 89 de [10].

Para cada subconjunto $A \subset[0,1]$ e cada subintervalo $I=[a, b] \subset[0,1]$ denotaremos por $A_{I}$ o conjunto $\{(b-a) x+a: x \in A\}$. Notemos que, se $A$ for mensurável, então então $A_{I}$ é mensurável e $m\left(A_{I}\right)=(b-a) m(A)$, onde $m$ é a medida de Lebesgue.

Seguiremos agora com o principal resultados dessa seção. Para essa demonstração cons- 
truiremos uma família de conjuntos quase disjuntos no intervalo $[0,1]$ e usa-la para definirmos uma família de funções Lebesgue mensuráveis. Através de um resultado envolvendo bases de Schauder, veremos que essa família de funções gera um espaço vetorial fechado em $\mathcal{G}$.

Teorema 3.2.1. O Conjunto $\mathcal{G}$ é espaçável.

Demonstração. Vamos definir uma família de funções $g_{k} \in \mathcal{G}$. Para isso consideraremos subconjuntos $A_{j} \subset[0,1]$ da seguinte forma: O conjunto $A_{1}$ será um conjunto de cantor em $[0,1]$ com medida $2^{-1}$. E a partir disso definiremos $A_{2}:=\cup\left\{\left(A_{1}\right)_{I}: I\right.$ é uma buraco de $\left.A_{1}\right\}$, isto é, cada buraco de $A_{1}$ conterá uma cópia de $A_{1}$ (a essas cópias daremos do nome de Cantor componentes de $\left.A_{1}\right)$. Teremos então $A_{2}$ com Cantor componentes $\left(A_{1}\right)_{I}$ e, pela quantidade de buracos de $A_{1}$ ser enumerável, pelos buracos serem dois a dois disjuntos e definindo $B_{1}:=\left\{I \subset[0,1]: I\right.$ é buraco de $\left.A_{1}\right\}$, segue:

$$
\begin{aligned}
m\left(A_{2}\right) & =\sum_{I \in B_{1}} m\left(\left(A_{1}\right)_{I}\right) \\
& =\sum_{I \in B_{1}} m(I) m\left(A_{1}\right) \\
& =m\left(A_{1}\right) \sum_{I \in B_{1}} m(I) \\
& =m\left(A_{1}\right) m\left([0,1] \backslash A_{1}\right) \\
& =\left(2^{-1}\right)\left(2^{-1}\right)=2^{-2} .
\end{aligned}
$$

Indutivamente, para $j \geq 3$ suponha que $A_{j}=\cup\left\{\left(A_{1}\right)_{I}: I\right.$ é um buraco de uma Cantor componente de $\left.A_{j-1}\right\}$ e que $m\left(A_{j}\right)=2^{-j}$. Então defina:

$$
A_{j+1}:=\cup\left\{\left(A_{1}\right)_{I}: I \text { é um buraco de uma Cantor componente de } A_{j}\right\} \text {. }
$$

Assim, $A_{j+1}$ possui Cantor componentes $\left(A_{1}\right)_{I}$ e, analogamente ao cálculo para $m\left(A_{2}\right)$, $m\left(A_{j+1}\right)=2^{-(j+1)}$. Obtemos então uma sequência $\left(A_{j}\right)$ de subconjuntos de $[0,1]$ que satisfazem as seguintes propriedades:

(i) $m\left(A_{j} \cap A_{k}\right)=0$;

(ii) $m\left(\cup_{j} A_{j}\right)=\sum_{j=1}^{\infty} m\left(A_{j}\right)=\sum_{j=1}^{\infty} 2^{-j}=1$;

(iii) Para todo subintervalo $J \subset[0,1]$, existe $j_{0} \in \mathbb{N}$ tal que para cada $j>j_{0}, A_{j}$ possui uma Cantor componente contida em $J$.

Para cada $k \in \mathbb{N}$ considere uma sequência estritamente crescente de naturais $\left(n_{k, j}\right)_{j}$ de modo que essas sequências sejam duas a duas disjuntas. Podemos assim definir, para cada $k$, as 
funções $g_{k}:[0,1] \rightarrow \mathbb{R}$ por:

$$
\left.g_{k}:=\sum_{j=1}^{\infty} \theta^{n_{k, j}} \chi_{A_{n_{k, j}}}, \text { com } \theta \in\right] 1,2[
$$

As $g_{k}$ são Lebesgue integráveis pois, pelo Teorema da Convergência Monótona (veja [4], página 31) segue:

$$
\int g_{k}=\sum_{j=1}^{\infty} \int \theta^{n_{k, j}} \chi_{A_{n_{k, j}}}=\sum_{j=1}^{\infty}\left(\frac{\theta}{2}\right)^{n_{k, j}}<\sum_{n=1}^{\infty}\left(\frac{\theta}{2}\right)^{n}=\frac{\theta}{2-\theta}<\infty .
$$

Se tomarmos um intervalo qualquer $J \subset[0,1]$, pela propriedade (iii), existe $j_{0}$ tal que para todo $j>j_{0}, A_{j}$ tem uma Cantor componente contida em $J$ e assim as $g_{k}$ não são limitadas na união dessas componentes. Vimos acima que a medida de cada componente não é nula e, portanto, $g_{k} \in \mathcal{G}$ para todo $k$.

Finalmente, para concluirmos a espaçabilidade de $\mathcal{G}$, vamos mostrar que $\overline{\operatorname{span}\left\{g_{k}: k \in \mathbb{N}\right\}}$ $\subset \mathcal{G} \cup\{0\}$. Afirmo que, para cada natural $m_{1}<m_{2}$ e $a_{1}, \ldots, a_{m_{2}} \in \mathbb{R},\left\|\sum_{k=1}^{m_{1}} a_{k} g_{k}\right\| \leq\left\|\sum_{k=1}^{m_{2}} a_{k} g_{k}\right\|$. De fato, como os suportes das $g_{k}$ são dois a dois disjuntos quase sempre então vale para cada $m \in \mathbb{N}:$

$$
\left\|\sum_{k=1}^{m} a_{k} g_{k}\right\|=\int\left|\sum_{k=1}^{m} a_{k} g_{k}\right|=\int \sum_{k=1}^{m}\left|a_{k} g_{k}\right|
$$

Assim, para naturais $m_{1}<m_{2}$ segue:

$$
\left\|\sum_{k=1}^{m_{1}} a_{k} g_{k}\right\|=\int \sum_{k=1}^{m_{1}}\left|a_{k} g_{k}\right| \leq \int \sum_{k=1}^{m_{2}}\left|a_{k} g_{k}\right|=\left\|\sum_{k=1}^{m_{2}} a_{k} g_{k}\right\| \text {. }
$$

Da desigualdade acima e pelo Teorema 1.4.11 $\left(g_{k}\right)_{k}$ é sequência básica e, portanto, base de Schauder para $\overline{\operatorname{span}\left\{g_{k}: k \in \mathbb{N}\right\}}$. Se tomarmos então $F \in \overline{\operatorname{span}\left\{g_{k}: k \in \mathbb{N}\right\}}$ não nulo, teremos $F=\sum_{k=1}^{\infty} \alpha_{k} g_{k} \operatorname{com}\left(\alpha_{k}\right)_{k}$ uma sequência de números reais não todos nulos e então para um intervalo aberto $J \subset[0,1]$ podemos tomar um $\alpha_{k_{0}} \neq 0$ e existirá, pela propriedade (iii), um $j_{0}$ tal que uma Cantor componente $B_{j}$ de $A_{n_{k_{0}, j}}$ está contida em $J$ para todo $j>j_{0}$. Como cada $B_{j}$ tem medida positiva e $\left.F\right|_{B_{j}} \equiv \theta^{n_{k_{0}, j}}, F$ não é essencialmente limitada em $J$ e, pela arbitrariedade de $J$, concluímos que $F \in \mathcal{G}$. Logo, $\mathcal{G}$ é espaçável.

Pelas funções Riemann integráveis serem limitadas, segue o interessante:

Corolário 3.2.2. O Conjuntos das Funções Lebesgue integráveis em [0,1] que não equivalem a uma função Riemann integrável em nenhum subintervalo de seu domínio é espaçável. 


\section{Capítulo 4}

\section{Funcionais}

Nos capítulos anteriores vimos resultados de lineabilidade e espaçabilidade em conjuntos de funções com o domínio em $\mathbb{R}$. Porém, também podemos trabalhar esses conceitos em conjuntos de funcionais lineares em um espaço vetorial $X$. Alguns resultados de lineabilidade em conjuntos de funcionais lineares foram apresentados em [1] e [8]. Neste capítulo detalharemos as demonstrações desses resultados.

\subsection{Funções aditivas descontínuas e lineares}

Já vimos no Capítulo 2 alguns teoremas do artigo [8] de García-Pacheco, Palmberg e Seoane-Sepúlveda. Nessa seção veremos mais dois resultados desse artigo que foram colocados nesse capítulo por se relacionarem (ou se aproximarem) à lineabilidade de conjuntos de funcionais lineares.

O primeiro teorema fala sobre funções aditivas descontínuas. Para $X$ e $Y$ espaços vetoriais reais, uma função $f: X \rightarrow Y$ é aditiva se $f(x+y)=f(x)+f(y)$ para todo $x, y \in X$. Para $\mathbb{K}$ um subcorpo de $\mathbb{R}$, chamaremos $f$ de $\mathbb{K}$-linear se for aditiva e satisfazer $f(k x)=k f(x)$ para cada $x \in X$ e $k \in \mathbb{K}$. Caso $\mathbb{K}=\mathbb{R}$ dizemos que $f$ é, apenas, linear.

Toda função aditiva também é $\mathbb{Q}$-linear:

Seja $\frac{p}{q} \in \mathbb{Q}$ onde $p, q \in \mathbb{Z}$ e $q \neq 0$. Da aditividade de $f$ temos que $p f(x)=f(p x)$ para todo $x \in X$. Logo, basta verificar que $\left(\frac{1}{q}\right) f(x)=f\left(\frac{x}{q}\right)$.

$$
\left(\frac{1}{q}\right) f(x)=\left(\frac{1}{q}\right) f\left(\frac{x q}{q}\right)=q \frac{1}{q} f\left(\frac{x}{q}\right)=f\left(\frac{x}{q}\right) .
$$

Logo f é $\mathbb{Q}$-linear.

Um resultado interessante nos mostra o comportamento de funções aditivas contínuas de $\mathbb{R}$ em $\mathbb{R}$. 
Proposição 4.1.1. Uma função $f: \mathbb{R} \rightarrow \mathbb{R}$ é aditiva contínua se, somente se, para todo $x \in \mathbb{R}, f(x)=\alpha x$, em que $\alpha \in \mathbb{R}$.

Demonstração. Se $f$ é aditiva e contínua então tome $x \in \mathbb{R}$ arbitrário e uma sequência de números racionais $\left(q_{n}\right)$ que converge para $x$. Como $f$ é contínua temos que $f\left(q_{n}\right)$ converge para $f(x)$. Pela aditividade de $f\left(q_{n}\right)$ temos:

$$
f\left(q_{n}\right)=q_{n} f(1)
$$

Como os limites de sequências em $\mathbb{R}$ são únicos, concluímos então:

$$
f(x)=x f(1)=\alpha x
$$

A outra implicação é clara pois a função identidade é contínua e teremos portanto um produto de uma função contínua por uma constante.

Esse resultado nos leva a imaginar que toda função aditiva de $\mathbb{R}$ em $\mathbb{R}$ é assim, ou senão, que há poucas funções com comportamento diferente desse. Há um exemplo de função aditiva descontínua em [10], página 33. No entanto o próximo teorema nos mostra que o conjunto das funções aditivas descontínuas é grande ao ponto de conter um espaço de vetorial com dimensão infinita.

Para essa demonstração, relembremos que um espaço vetorial topológico é um espaço vetorial munido de uma topologia de Hausdorff cujas operações de soma e produto por escalar são funções contínuas. Para chegar no resultado, consideraremos uma uma base de Hamel para $\mathbb{R}$ como espaço vetorial sobre $\mathbb{Q}$. A partir disso construímos uma família de funções que, por um processo parecido com a demonstração da Proposição 4.1.1, veremos gerar funções lineares (portanto aditivas). E por argumento de contradição teremos que essas funções geradas são descontínuas.

Teorema 4.1.2. Em todo espaço vetorial topológico real, o conjunto das funções aditivas descontínuas com valores reais é lineável.

Demonstração. Dado um espaço vetorial topológico $X$, vamos construir um espaço vetorial real com dimensão $\mu:=\max \{c, \operatorname{dim}(X)\}$ tal que todo elemento não nulo é uma função aditiva descontínua de $X$ em $\mathbb{R}$.

Considere os seguintes conjuntos:

$$
\begin{gathered}
L:=\{f: X \rightarrow \mathbb{R} \mid f \text { é aditiva }\}, \\
L_{c}:=\{f: X \rightarrow \mathbb{R} \mid f \text { é aditiva e contínua }\} .
\end{gathered}
$$


$L$ é um espaço vetorial real e $L_{c}$ é subespaço de $L$.

Seja $V$ uma base de $X$ como espaço vetorial real e defina $B:=\{\gamma v: \gamma \in \mathcal{H}, v \in V\}$, onde $\mathcal{H}$ é a base de Hamel para $\mathbb{R}$ como espaço vetorial sobre $\mathbb{Q}$. Assim, $B$ é base de $X$ como espaço vetorial sobre $\mathbb{Q}$ de cardinalidade $\mu$. Para cada $b \in B$ podemos definir a função $f_{b}: B \rightarrow \mathbb{Q}$ por:

$$
\bar{f}_{b}(\xi):= \begin{cases}1 & \text { se } \xi=b \\ 0 & \text { se } \xi \neq b .\end{cases}
$$

Então, seja $f_{b}$ a extensão linear de $\bar{f}_{b}$ em $X$ e considere o conjunto $L_{s}:=\operatorname{span}\left\{f_{b}: b \in B\right\}$.

Temos que $\left\{f_{b}: b \in B\right\}$ é linearmente independente, pois:

$$
\sum_{i=1}^{k} \alpha_{i} f_{b_{i}}=0 \Rightarrow \sum_{i=1}^{k} \alpha_{i} f_{b_{i}}\left(b_{j}\right)=0 \text { para todo } j \in\{1, \ldots, k\} \Rightarrow \alpha_{1}=\ldots=\alpha_{k}=0
$$

com $\mathrm{k} \in \mathbb{N}$. Logo $L_{s}$ é um espaço vetorial de dimensão $\mu$.

Agora, vamos mostrar que $L_{s}$ é um subespaço de $L$ que não intercepta $L_{c}$ a menos do $\{0\}$. Seja $F=\sum_{i=1}^{k} \alpha_{i} f_{b_{i}}$ um elemento de $L_{s}$, em que cada $a_{i} \in \mathbb{R} \backslash\{0\}$. Suponha, por absurdo, que $F$ seja contínua. Então a restrição de $F$ em $\mathbb{R} b_{1}$ é uma função contínua. Como $X$ é um espaço vetorial topológico, a operação de produto por escalar é contínua, portanto, para $r \in \mathbb{R}$ qualquer e $\left(q_{n}\right) \subset \mathbb{Q}$ uma sequência tal que $q_{n} \underset{n \rightarrow \infty}{\longrightarrow} r$, temos:

$$
q_{n} \rightarrow r \Rightarrow b_{1} q_{n} \rightarrow b_{1} r \Rightarrow F\left(b_{1} q_{n}\right) \rightarrow F\left(b_{1} r\right)
$$

Como $F$ é combinação linear de funções aditivas (as $f_{i}$ ), então $F$ é aditiva. Pelo que foi visto anteriormente, $F$ também é $\mathbb{Q}$-linear e, portanto, $F\left(b_{1} q_{n}\right)=q_{n} F\left(b_{1}\right) \forall n \in \mathbb{N}$. Como $q_{n} F\left(b_{1}\right) \rightarrow r F\left(b_{1}\right)$ e todo espaço vetorial topológico é um espaço de Hausdorff, temos que $r F\left(b_{1}\right)=F\left(r b_{1}\right)$. Pela arbitrariedade de $r$ podemos concluir que $F$ é linear. Também concluímos que $\left.F\right|_{\mathbb{R} b_{1}}$ só se anula no zero, pois $F\left(r b_{1}\right)=r F\left(b_{1}\right)=r\left(\sum_{i=1}^{k} \alpha_{i} f_{b_{i}}\left(b_{1}\right)\right)=r \alpha_{1}$, onde $r \in \mathbb{R}$ e $\alpha_{1} \neq 0$. Por outro lado, existem $\gamma \in \mathcal{H}$ e $v \in V$ tal que $b_{1}=\gamma v$. Notemos que $\mathcal{H} v=\{h v: h \in \mathcal{H}\} \subset B$ e então tomemos $\tilde{b} \in \mathcal{H} v \backslash\left\{b_{i}\right\}_{i=1}^{k}$. Temos que $\tilde{b} \in \mathbb{R} b_{1}$ pois $\tilde{b}=h v=h \gamma^{-1} b_{1}$ e, portanto, $\left.F\right|_{\mathbb{R} b_{1}}(\tilde{b})=\sum_{i=1}^{k} \alpha_{i} f_{b_{i}}(\tilde{b})=0$. Chegamos então numa contradição, pois $\left.F\right|_{\mathbb{R} b_{1}}$ só se anularia em 0 e $\tilde{b} \neq 0$, já que $0 \notin B$. Concluímos então que $F$ é descontínua, e como $F$ é arbitrária, $L_{s}$ está contido em $\left(L \backslash L_{c}\right) \cup\{0\}$, isto é, $L \backslash L_{c}$ é lineável.

O segundo resultado do artigo estudado trata de funcionais lineares num espaço normado com dimensão infinita. Porém o foco deste teorema são as funções descontínuas destacando que o conjunto dessas funções, usualmente menos explorado na análise funcional, possuí uma quantidade imensa de elementos. 
Nessa demonstração tomaremos uma família de funções definidas a partir de um sub conjunto enumerável da base do espaço. Mostraremos que essa família gera um subespaço de dimensão infinita montando uma matriz de Vandermonde. E então utilizaremos a noção de continuidade atrelada ao conceito de funcionais limitados em espaços normados.

Teorema 4.1.3. Em todo espaço normado real de dimensão infinita, o conjunto de todas as funções reais lineares descontínuas é lineável.

Demonstração. Seja $X$ um espaço vetorial real normado de dimensão infinita. Tome um conjunto infinito enumerável linearmente independente $B:=\left\{v_{j}\right\}_{j=0}^{\infty}$, contido na esfera $S_{X}=$ $\{x \in X:|x|=1\}$. Para cada $i \in \mathbb{N}$, com $i \geq 2$, podemos definir uma função $\tilde{f}_{i}: B \rightarrow \mathbb{R}$ por:

$$
\tilde{f}_{i}\left(v_{j}\right):=i^{j}
$$

Completando o conjunto $B$ para uma base $B^{\prime}$ de $X$, a função $\tilde{f}_{i}$ pode então ser estendida linearmente para todo o espaço. Denotaremos essa extensão por $f_{i}$.

Definimos agora o espaço vetorial $V:=\operatorname{span}\left\{f_{i}: i \in \mathbb{N}, i \geq 2\right\}$ e notemos que cada elemento de $V$ é um funcional linear (pois é combinação linear de lineares). Veremos que $\left\{f_{i}: i \in \mathbb{N}, i \geq 2\right\}$ é base para $V$.

Se $\sum_{j=1}^{k} \alpha_{j} f_{i_{j}}=0$, onde $k \in \mathbb{N},\left\{\alpha_{j}\right\}_{j=1}^{k} \subset \mathbb{R}$ e $i_{1}>\cdots>i_{k} \geq 2$, então aplicando em cada $v_{0}, \ldots, v_{k-1}$ obtemos o seguinte sistema:

$$
\left\{\begin{array}{ccccccc}
\alpha_{1} f_{i_{1}}\left(v_{0}\right) & + & \ldots & + & \alpha_{k} f_{i_{k}}\left(v_{0}\right) & = & 0 \\
\alpha_{1} f_{i_{1}}\left(v_{1}\right) & + & \ldots & + & \alpha_{k} f_{i_{k}}\left(v_{1}\right) & = & 0 \\
\vdots & & \vdots & & \vdots & & \vdots \\
\alpha_{1} f_{i_{1}}\left(v_{k-1}\right) & + & \ldots & + & \alpha_{k} f_{i_{k}}\left(v_{k-1}\right) & & 0
\end{array}\right.
$$

E então, passando para a notação de matrizes:

$$
\left(\begin{array}{ccccc}
1 & 1 & 1 & \ldots & 1 \\
i_{1} & i_{2} & i_{3} & \ldots & i_{k} \\
i_{1}^{2} & i_{2}^{2} & i_{3}^{2} & \ldots & i_{k}^{2} \\
\vdots & \vdots & \vdots & \ddots & \vdots \\
i_{1}^{k-1} & i_{2}^{k-1} & i_{3}^{k-1} & \ldots & i_{k}^{k-1}
\end{array}\right)\left(\begin{array}{c}
\alpha_{1} \\
\alpha_{2} \\
\alpha_{3} \\
\vdots \\
\alpha_{k}
\end{array}\right)=\left(\begin{array}{c}
0 \\
0 \\
0 \\
\vdots \\
0
\end{array}\right)
$$

Notamos que a matriz acima é de Vandermonde e, portanto, seu determinante $\prod_{1 \leq n<m \leq k}\left(i_{m}-\right.$ $i_{n}$ ) é não nulo pois os $i_{j}$ são distintos. Assim, a matriz acima é invertível e a única solução para o sistema é a trivial, isto é, $\alpha_{1}=\cdots=\alpha_{k}=0$. Logo, o conjunto $\left\{f_{i}: i \in \mathbb{N}, i \geq 2\right\}$ é linearmente independente e $\operatorname{dim}(V)=\aleph_{0}$.

Agora tome $F=\sum_{h=1}^{k} \alpha_{h} f_{i_{h}}$ um elemento qualquer de $V$, onde $k \in \mathbb{N},\left\{\alpha_{h}\right\}_{h=1}^{k} \subset \mathbb{R} \backslash\{0\}$ 
e $i_{1}>\cdots>i_{k} \geq 2$. Para cada $j \in \mathbb{N}$ nós temos:

$$
\begin{aligned}
\left|F\left(v_{j}\right)\right| & =\left|\alpha_{1} i_{1}^{j}+\cdots+\alpha_{k} i_{k}^{j}\right| \\
& \geq\left|\alpha_{1}\right| i_{1}^{j}-\left|\alpha_{2}\right| i_{2}^{j}-\cdots-\left|\alpha_{k}\right| i_{k}^{j}
\end{aligned}
$$

pela desigualdade triangular, já que:

$$
\begin{aligned}
\left|\alpha_{1}\right| i_{1}^{j} & =\left|\alpha_{1} i_{1}^{j}+\alpha_{2} i_{2}^{j}+\cdots+\alpha_{k} i_{k}^{j}-\alpha_{2} i_{2}^{j}-\cdots-\alpha_{k} i_{k}^{j}\right| \\
& \leq\left|\alpha_{1} i_{1}^{j}+\cdots+\alpha_{k} i_{k}^{j}\right|+\left|\alpha_{2} i_{2}^{j}\right|+\cdots+\left|\alpha_{k} i_{k}^{j}\right| .
\end{aligned}
$$

Como $\left|\alpha_{1}\right| i_{1}^{j}=\frac{(k-1)\left|\alpha_{1}\right| i_{1}^{j}}{k-1}$, temos também:

$$
\begin{aligned}
\left|F\left(v_{j}\right)\right| & \geq\left|\alpha_{1}\right| i_{1}^{j}-\left|\alpha_{2}\right| i_{2}^{j}-\cdots-\left|\alpha_{k}\right| i_{k}^{j} \\
& =\left(\frac{\left|\alpha_{1}\right|}{k-1} i_{1}^{j}-\left|\alpha_{2}\right| i_{2}^{j}\right)+\cdots+\left(\frac{\left|\alpha_{1}\right|}{k-1} i_{1}^{j}-\left|\alpha_{k}\right| i_{k}^{j}\right) .
\end{aligned}
$$

Para cada $h=2, \ldots, k$, notamos:

$$
\left(\frac{\left|\alpha_{1}\right|}{k-1} i_{1}^{j}-\left|\alpha_{h}\right| i_{h}^{j}\right)=i_{h}^{j}\left(\frac{\left|\alpha_{1}\right|}{k-1}\left(\frac{i_{1}}{i_{h}}\right)^{j}-\left|\alpha_{h}\right|\right)
$$

e, como $\frac{i_{1}}{i_{h}}>1$, concluímos que quando $j \rightarrow \infty,\left(\frac{\left|\alpha_{1}\right|}{k-1} i_{1}^{j}-\left|\alpha_{h}\right| i_{1}^{j}\right) \rightarrow \infty$ para cada $h \in$ $\{2, \ldots, k\}$. Logo $F$ é um funcional não limitado em $S_{X}$ e, portanto, $F$ não é contínua. Pela arbitrariedade de $F$ concluímos que $V$ é um espaço vetorial de dimensão $\aleph_{0}$ contido no conjunto das funções lineares de $X$ com valores em $\mathbb{R}$ descontínuas.

\subsection{Funcionais lineares que atingem a norma}

Nos resultados da seção anterior trabalhamos com funções aditivas e funcionais lineares descontínuos. Os protagonistas agora são os funcionais lineares contínuos. Estudaremos aqui dois resultados que foram apresentados por Acosta, Aizpuru, Aron, García-Pacheco, e outros autores em [1] sobre a linearidade do conjunto de funcionais que atingem a sua norma. Vale a pena então fazermos algumas definições para prosseguirmos.

Seja $X$ um espaço de Banach. Dizemos que um funcional linear $f \in X^{*}$ atinge a norma se existe $x \in B_{X}$ tal que $|f(x)|=\|f\|$. Denotaremos por $N A(X)$ o conjunto dos funcionais de $X^{*}$ que atingem a norma.

O primeiro teorema que iremos ver fala sobre uma condição que um espaço de Banach $X$ deve obedecer para que $N A(X)$ seja lineável. É recomendável a leitura da seção 1.4 pois os conceitos apresentados lá serão amplamente usados nas próximas demonstrações. 
Vale notar que na ao invés da construção de uma família de funções, no próximo teorema usaremos os funcionais coeficientes a lineabilidade de $N A(X)$ para gerar o espaço vetorial buscado.

Teorema 4.2.1. Se $X$ é um espaço de Banach com base de Schauder monótona, então $N A(X)$ é lineável.

Demonstração. Seja $\left(x_{n}\right)_{n \in \mathbb{N}}$ uma base de Schauder para $X$ e $M=\operatorname{span}\left\{x_{n}^{*}: n \in \mathbb{N}\right\} \subset$ $X^{*}$. A Proposição 1.4.15 nos mostrou que $\left\{x_{n}^{*}: n \in \mathbb{N}\right\}$ é sequência básica e, portanto, linearmente independente. $\operatorname{Logo} \operatorname{dim} M=\aleph_{0}$.

Agora verificaremos que $M \subset N A(X)$ : Tome $x^{*}=\sum_{n=1}^{m} \alpha_{n} x_{n}^{*} \in M$ qualquer e seja $P_{m}$ a $m$-ésima projeção natural associada a base $\left(x_{n}\right)_{n \in \mathbb{N}}$. Aplicando a transposta de $P_{m}$ em $x^{*}$ temos:

$$
\begin{aligned}
P_{m}^{t}\left(x^{*}\right)(x) & =x^{*}\left(P_{m}\left(\sum_{n=1}^{\infty} \beta_{n} x_{n}\right)\right)=x^{*}\left(\sum_{n=1}^{m} \beta_{n} x_{n}\right) \\
& =\sum_{n=1}^{m} \alpha_{n} x_{n}^{*}\left(\sum_{n=1}^{m} \beta_{n} x_{n}\right)=\sum_{n=1}^{m} \alpha_{n} \beta_{n} \\
& =x^{*}\left(\sum_{n=1}^{\infty} \beta_{n} x_{n}\right)=x^{*}(x),
\end{aligned}
$$

onde $x \in M$ é arbitrário. Concluímos então que $P_{m}^{t}\left(x^{*}\right)=x^{*}$. E assim:

$$
\begin{aligned}
\left\|x^{*}\right\| & =\sup \left\{x^{*}(x): x \in B_{X}\right\}=\sup \left\{P_{m}^{t}\left(x^{*}\right)(x): x \in B_{X}\right\} \\
& =\sup \left\{x^{*}\left(P_{m}(x)\right): x \in B_{X}\right\} .
\end{aligned}
$$

Como a base de $X$ é monótona, pela Proposição 1.4.10, $P_{m}\left(B_{X}\right)=B_{\mathrm{Span}\left\{x_{1}, \ldots, x_{m}\right\}}$. E portanto:

$$
\left\|x^{*}\right\|=\sup \left\{x^{*}\left(P_{m}(x)\right): x \in B_{X}\right\}=\sup \left\{x^{*}(x): x \in B_{\operatorname{Span}\left\{x_{1}, \ldots, x_{m}\right\}}\right\} .
$$

Sabe-se que a bola fechada num espaço de dimensão finita é compacto (vide [13] páginas 77 e 78), logo existe um $x_{0} \in B_{\operatorname{Span}\left\{x_{1}, \ldots, x_{m}\right\}}$ tal que $x^{*}\left(x_{0}\right)=\sup \left\{x^{*}(x): x \in\right.$ $\left.B_{\mathrm{Span}\left\{x_{1}, \ldots, x_{m}\right\}}\right\}=\left\|x^{*}\right\|$. Segue que $M \subset N A(X)$, e, portanto, $N A(X)$ é lineável.

Finalmente, o segundo resultado apresentado em [1] envolve um trabalho com espaços complementados. Lembremos então essa definição para seguirmos adiante.

Definição 4.2.2. Um subespaço $M$ de um espaço vetorial topológico $X$ é complementado em $X$ se é fechado em $X$ e existe um subespaço fechado $N$ em $X$ tal que $X=M \oplus N$.

Vimos na seção 1.4 a definição de uma outra norma em espaços de Banach com base de Schauder. Essa ferramenta será importante no próximo teorema. Veremos que, se tivermos 
um espaço de Banach com base de Schaulder, porém, sem base monótona, ainda teremos a linabilidade do conjunto dos funcionais que atingem a norma. Nessa demonstração renormaremos o espaços objetivando a lineabilidade do conjunto $N A(X)$, para um espaço de Banach $X$.

Teorema 4.2.3. Seja $X$ um espaço de Banach. Se $Y \subset X$ é um subespaço fechado de dimensão infinita com base de Schauder complementado em X, então existe uma norma equivalente ||$|\cdot|||$ em $X$ tal que $N A(X, \||\cdot|||)$ é lineável.

Demonstração. Seja $\left(y_{n}\right)$ uma base de Schauder de $Y$. Por ser fechado $Y$ é espaço de Banach com norma herdada de $X$. Pela Proposição 1.4 .8 o espaço $\left(Y,\|\cdot\|_{\left(y_{n}\right)}\right)$ tem base de Schauder monótona e portanto, pelo Teorema 4.2.1, $N A\left(Y,\|\cdot\|_{\left(y_{n}\right)}\right)$ é lineável. Vamos então definir uma norma \|\|$\cdot \| l X \rightarrow \mathbb{R}$ tal que se restrita a $Y$ equivalha a norma $\|\cdot\|_{\left(y_{n}\right)}$ e mostrar que $N A\left(Y,\|\cdot\|_{\left(y_{n}\right)}\right) \subset N A(X,\|\| \cdot\|\|)$ (Note que aqui há um abuso de notação e que na verdade encontraremos um subespaço de $N A(X,\||\cdot|\|)$ isométrico a $\left.N A\left(Y,\|\cdot\|_{\left(y_{n}\right)}\right)\right)$.

Por hipótese, $Y$ é complementado em $X$ portanto existe um subespaço $Z \subset X$ fechado o qual, para todo $x \in X$, existem únicos $y \in Y$ e $z \in Z$ tais que $x=y+z$. Portanto definiremos, para cada $x \in X,\|\| x \|:=\sqrt{\|y\|_{\left(y_{n}\right)}^{2}+\|z\|^{2}}$, onde $\|\cdot\|$ é a norma de $Z$ herdada de $X$. Observemos que \|\|$\cdot \| \mid$ é de fato uma norma em $X$, pois:

- Para $x=y+z \in X$ qualquer:

$$
\begin{aligned}
\||x|\|=0 & \Longleftrightarrow\|y\|_{\left(y_{n}\right)}^{2}+\|z\|^{2}=0 \\
& \Longleftrightarrow\|y\|_{\left(y_{n}\right)}=\|z\|=0 \\
& \Longleftrightarrow y=z=0 \\
& \Longleftrightarrow x=0 ;
\end{aligned}
$$

- Para $x=y+z \in X$ e $\lambda \in \mathbb{K}$ quaisquer:

$$
\begin{aligned}
\|\lambda x\| & =\sqrt{\|\lambda y\|_{\left(y_{n}\right)}^{2}+\|\lambda z\|^{2}} \\
& =\sqrt{|\lambda|^{2}\left(\|y\|_{\left(y_{n}\right)}^{2}+\|z\|^{2}\right)} \\
& =|\lambda| \sqrt{\|y\|_{\left(y_{n}\right)}^{2}+\|z\|^{2}} \\
& =|\lambda| \mid\|x\| \| ;
\end{aligned}
$$

- Para $x_{1}=y_{1}+z_{1}, x_{2}=y_{2}+z_{2} \in X$ quaisquer:

$$
\begin{aligned}
& \left(\left\|y_{1}\right\|_{\left(y_{n}\right)}\left\|z_{2}\right\|-\left\|z_{1}\right\|\left\|y_{2}\right\|_{\left(y_{n}\right)}\right)^{2} \geq 0 \\
\Rightarrow \quad & \left\|y_{1}\right\|_{\left(y_{n}\right)}^{2}\left\|z_{2}\right\|^{2}+\left\|z_{1}\right\|^{2}\left\|y_{2}\right\|_{\left(y_{n}\right)}^{2} \geq 2\left\|y_{1}\right\|_{\left(y_{n}\right)}\left\|y_{2}\right\|_{\left(y_{n}\right)}\left\|z_{1}\right\|\left\|z_{2}\right\| .
\end{aligned}
$$


Segue:

$$
\begin{aligned}
\left\|\left|x_{1}\right|\right\|\|\| x_{2} \mid \| & =\sqrt{\left(\left\|y_{1}\right\|_{\left(y_{n}\right)}^{2}+\left\|z_{1}\right\|^{2}\right)\left(\left\|y_{2}\right\|_{\left(y_{n}\right)}^{2}+\left\|z_{2}\right\|^{2}\right)} \\
& =\sqrt{\left\|y_{1}\right\|_{\left(y_{n}\right)}^{2}\left\|y_{2}\right\|_{\left(y_{n}\right)}^{2}+\left\|y_{1}\right\|_{\left(y_{n}\right)}^{2}\left\|z_{2}\right\|^{2}+\left\|z_{1}\right\|^{2}\left\|y_{2}\right\|_{\left(y_{n}\right)}^{2}+\left\|z_{1}\right\|^{2}\left\|z_{2}\right\|^{2}} \\
& \geq \sqrt{\left\|y_{1}\right\|_{\left(y_{n}\right)}^{2}\left\|y_{2}\right\|_{\left(y_{n}\right)}^{2}+2\left\|y_{1}\right\|_{\left(y_{n}\right)}\left\|y_{2}\right\|_{\left(y_{n}\right)}\left\|z_{1}\right\|\left\|z_{2}\right\|+\left\|z_{1}\right\|^{2}\left\|z_{2}\right\|^{2}} \\
& =\sqrt{\left(\left\|y_{1}\right\|_{\left(y_{n}\right)}\left\|y_{2}\right\|_{\left(y_{n}\right)}+\left\|z_{1}\right\|\left\|z_{2}\right\|\right)^{2}} \\
& =\left\|y_{1}\right\|_{\left(y_{n}\right)}\left\|y_{2}\right\|_{\left(y_{n}\right)}+\left\|z_{1}\right\|\left\|z_{2}\right\| .
\end{aligned}
$$

Portanto ||$\left|x_{1}\right|||||\left|x_{2}\right||| \geq\left\|y_{1}\right\|_{\left(y_{n}\right)}\left\|y_{2}\right\|_{\left(y_{n}\right)}+\left\|z_{1}\right\|\left\|z_{2}\right\|$. Disso decorre:

$$
\begin{aligned}
\left.\left\|x_{1}+x_{2}\right\|\right|^{2} & =\left\|y_{1}+y_{2}\right\|_{\left(y_{n}\right)}^{2}+\left\|z_{1}+z_{2}\right\|^{2} \\
& \leq\left\|y_{1}\right\|_{\left(y_{n}\right)}^{2}+2\left\|y_{1}\right\|_{\left(y_{n}\right)}\left\|y_{2}\right\|_{\left(y_{n}\right)}+\left\|y_{2}\right\|_{\left(y_{n}\right)}^{2}+\left\|z_{1}\right\|^{2}+2\left\|z_{1}\right\|\left\|z_{2}\right\|+\left\|z_{2}\right\|^{2} \\
& =\left\|y_{1}\right\|_{\left(y_{n}\right)}^{2}+\left\|z_{1}\right\|^{2}+\left\|y_{2}\right\|_{\left(y_{n}\right)}^{2}+\left\|z_{2}\right\|^{2}+2\left(\left\|y_{1}\right\|_{\left(y_{n}\right)}\left\|y_{2}\right\|_{\left(y_{n}\right)}+\left\|z_{1}\right\|\left\|z_{2}\right\|\right) \\
& =\|\| x_{1}\left\|\left.\right|^{2}+\right\|\left|x_{2} \|\right|^{2}+2\left(\left\|y_{1}\right\|_{\left(y_{n}\right)}\left\|y_{2}\right\|_{\left(y_{n}\right)}+\left\|z_{1}\right\|\left\|z_{2}\right\|\right) \\
& \leq\left\|\left|\left\|x_{1}\right\|\right|^{2}+\right\|\left|x_{2}\left\|\left.\right|^{2}+2 \mid\right\| x_{1}\|\|\left\|x_{2}\right\| \|\right. \\
& =\left(\left\|\left|x _ { 1 } \left\|\left|+\left\|\mid x_{2}\right\|\right)^{2} .\right.\right.\right.\right.
\end{aligned}
$$

$\operatorname{Logo}||\left|x_{1}+x_{2}\right|\|\leq\||| x_{1}|\|+||| x_{2}|| \mid$.

Veremos agora que $N A\left(Y,\|\cdot\|_{\left(y_{n}\right)}\right) \subset N A\left(X,|\|\cdot \mid\|)\right.$. Tomemos então um $f \in\left(Y,\|\cdot\|_{\left(y_{n}\right)}\right)^{*}$ qualquer e considere $\tilde{f}:(X,|\| \cdot|||) \rightarrow \mathbb{R}$ a extensão de $f$ em $X$ definida por $\tilde{f}(x)=\tilde{f}(y+z)=$ $f(y)$. Vejamos que $\tilde{f} \in(X,|||\cdot|||)^{*}$ :

- $\tilde{f}$ é claramente linear pois $f$ é.

- Para todo $x=y+z \in X \backslash\{0\}$ temos:

$$
\left\|| | x \left|\left\|=\left|\|y+z \mid\|=\sqrt{\|y\|_{\left(y_{n}\right)}^{2}+\|z\|^{2}} \geq\|y\|_{\left(y_{n}\right)},\right.\right.\right.\right.
$$

e portanto vale:

$$
|\tilde{f}(x)|=|f(y)| \leq\|f\|_{\left(y_{n}\right)}\|y\|_{\left(y_{n}\right)} \leq\|f\|_{\left(y_{n}\right)} \mid\|x\| \| \text {, para todo } x=y+z \in X, .
$$

Implicando $\tilde{f}$ ser limitada e $\|\tilde{f}\|\left\|:=\sup _{x \in S_{(X,\|\| \cdot\|\mid\|)}}|\tilde{f}(x)| \leq\right\| f \|_{\left(y_{n}\right)}$.

Na verdade vale $\|\mid \tilde{f}\|\|=\| f \|_{\left(y_{n}\right)}$. Acabamos de ver nas contas acima que $\|\tilde{f}\|\|\leq\| f \|_{\left(y_{n}\right)}$. Para a outra desigualdade considere que para todo $x \in X$ temos:

$$
|\tilde{f}(x)| \leq|| \tilde{f}|||||| x|| \mid
$$


Então particularmente para todo $y \in Y \subset X$ temos:

$$
|\tilde{f}(y)| \leq\|\tilde{f}|||||| y \mid\|
$$

ou seja:

$$
|f(y)| \leq\||\tilde{f}|\| \mid\|y\|_{\left(y_{n}\right)} .
$$

Portanto $\|f\|_{\left(y_{n}\right)} \leq\|\mid \tilde{f}\| \|$. Acarretando, junto a arbitrariedade de $f$, que $\|f\|_{\left(y_{n}\right)}=\|\| \tilde{f}\|\|$ para todo $f \in\left(Y,\|\cdot\|_{\left(y_{n}\right)}\right)^{*}$.

Se tomarmos então $g \in N A\left(Y,\|\cdot\|_{\left(y_{n}\right)}\right) \subset\left(Y,\|\cdot\|_{\left(y_{n}\right)}\right)^{*}$, existirá $y_{0} \in Y$ tal que $\left|g\left(y_{0}\right)\right|=$ $\|g\|_{\left(y_{n}\right)}:=\sup _{y \in S_{(Y,\|\cdot\|}\left(y_{n}\right)}|g(y)|$. Logo, para $\tilde{g} \in\left(X,|\|\cdot \mid\|)^{*}\right.$ extensão de $g$ :

$$
\left|\tilde{g}\left(y_{0}\right)\right|=\left|g\left(y_{0}\right)\right|=\|g\|_{\left(y_{n}\right)}=|||\tilde{g}||| .
$$

Isto é, $\tilde{g}$ atinge a norma em $y_{0} \in(X,\||\cdot|\|)$ e, portanto, $\tilde{g} \in N A(X,|||\cdot|||)$.

Consideremos então a identificação $I:\left(Y,\|\cdot\|_{\left(y_{n}\right)}\right)^{*} \rightarrow(X,\||\cdot|\| \mid)^{*}$, definida por $I(f)=\tilde{f}$ e que, pelo visto acima, é uma isometria. $\operatorname{Logo}, I\left(N A\left(Y,\|\cdot\|_{\left(y_{n}\right)}\right)\right)$ é uma cópia isométrica de $N A\left(Y,\|\cdot\|_{\left(y_{n}\right)}\right)$ em $(X,\|\| \cdot\|\|)^{*}$. Como $N A\left(Y,\|\cdot\|_{\left(y_{n}\right)}\right)$ é lineável, a sua cópia em $(X,\|\| \cdot\|\|)^{*}$ também será e, por $I\left(N A\left(Y,\|\cdot\|_{\left(y_{n}\right)}\right)\right) \subset N A(X,\||\cdot|\|), N A(X,|\|\cdot|\||)$ é lineável. 


\section{Referências Bibliográficas}

[1] Acosta, M.D., Aizpuru, A., Aron, R. e García-Pacheco, F.J.: Functionals that do not attain their norm. Bulletin of the Belgian Mathematical Society-Simon Stevin, 14(3):407418, 2007. 1, 51, 55, 56

[2] Aron, R., Gurariy, V.I. e Seoane, J.: Lineability and spaceability of sets of functions on $\mathbb{R}$. Proceedings of the American Mathematical Society, 133(3):795-803, 2005. 1, 35

[3] Aron, R. e Seoane-Sepúlveda, J.B.: Algebrability of the set of everywhere surjective functions on $\mathbb{C}$. Bulletin of the Belgian Mathematical Society-Simon Stevin, 14(1):2531, 2007. 1

[4] Bartle, R.G.: The Elements of Integration and Lebesgue Measure. John Wiley \& Sons, New York, 1995. 49

[5] Enflo, P., Gurariy, V.I. e Seoane-Sepúlveda, J.B.: On lineability and spaceability of sets in function spaces. preprint, 2002. 1

[6] Fonf, V.P., Gurariy, V.I. e Kadets, M.I.: An Infinite Dimensional Subspace of C (0, 1) Consisting of Nowhere Differentiable Functions. Dokladi na Bulgarskata Akademia na Naukite, 52(11-12):13-16, 1999. 1

[7] Gámez-Merino, J., Muñoz-Fernández, G., Sánchez, V. e Seoane-Sepúlveda, J.: Sierpinski-Zygmund functions and other problems on lineability. Proceedings of the American Mathematical Society, 138(11):3863-3876, 2010. 37

[8] García-Pacheco, F.J., Palmberg, N. e Seoane-Sepúlveda, J.: Lineability and algebrability of pathological phenomena in analysis. Journal of mathematical analysis and applications, 326(2):929-939, 2007. 1, 27, 32, 51

[9] García-Pacheco, F.J., Martín, M. e Seoane-Sepúlveda, J.: Lineability, spaceability, and algebrability of certain subsets of function spaces. Taiwanese Journal of Mathematics, 13(4):1257-1269, 2009. 47

[10] Gelbaum, B.R. e Olmsted, J.M.H.: Counterexamples in analysis. Courier Corporation, 2003. $35,47,52$

[11] Głab, S., Kaufmann, P. e Pellegrini, L.: Spaceability and algebrability of sets of nowhere integrable functions. Proceedings of the American Mathematical Society, 141(6):20252037, 2013. 1, 39, 47

[12] Gurariy, V.I: Subspaces and bases in spaces of continuous functions. Em Doklady Akademii Nauk, volume 167, páginas 971-973. Russian Academy of Sciences, 1966. 1 
[13] Kreyszig, E.: Introductory Functional Analysis with Applications. 5, 56

[14] Leoni, G.: A first course in Sobolev spaces. American Mathematical Soc., 2017.

[15] Lima, E.L.: Curso de análise. Número v. 1 em Projeto Euclides. Instituto de Matemática Pura e Aplicada, CNPq, 2009. 28

[16] Megginson, R.E.: An introduction to Banach space theory, volume 183. Springer Science \& Business Media, 2012. 4, 5, 6 